\title{
The molecular chemistry of diffuse and translucent clouds in the line-of-sight to Sgr B2: Absorption by simple organic and inorganic molecules in the GBT PRIMOS survey ${ }^{\star}$
}

\author{
J. F. Corby ${ }^{1,2}$, B. A. McGuire ${ }^{2}$, E. Herbst ${ }^{3}$, and A. J. Remijan ${ }^{2}$ \\ ${ }^{1}$ Department of Physics, University of South Florida, 4202 East Fowler Ave, Tampa, FL 33605, USA \\ e-mail: jfc2113@gmail.com \\ 2 National Radio Astronomy Observatory, 520 Edgemont Rd, Charlottesville, VA 22903, USA \\ 3 Departments of Chemistry and Astronomy, University of Virginia, McCormick Road, Charlottesville, VA 22904, USA
}

Received 13 April 2017 / Accepted 21 August 2017

\begin{abstract}
The 1-50 GHz PRebiotic Interstellar MOlecular Survey (PRIMOS) contains 50 molecular absorption lines observed in clouds located in the line-of-sight to Sgr B2(N). The line-of-sight material is associated with diffuse and translucent clouds located in the Galactic center, bar, and spiral arms in the disk. We measured the column densities and estimate abundances, relative to $\mathrm{H}_{2}$, of 11 molecules and additional isotopologues observed in this material. We used absorption by optically thin transitions of $c$ - $\mathrm{C}_{3} \mathrm{H}_{2}$ to estimate the molecular hydrogen columns, and argue that this method is preferable to more commonly used methods. We discuss the kinematic structure and abundance patterns of small molecules including the sulfur-bearing species $\mathrm{CS}, \mathrm{SO}, \mathrm{CCS}, \mathrm{H}_{2} \mathrm{CS}_{1}$ and $\mathrm{HCS}^{+}$; oxygen-bearing molecules $\mathrm{OH}, \mathrm{SiO}$, and $\mathrm{H}_{2} \mathrm{CO}$; and simple hydrocarbon molecules $c-\mathrm{C}_{3} \mathrm{H}_{2}, l-\mathrm{C}_{3} \mathrm{H}$, and $l-\mathrm{C}_{3} \mathrm{H}^{+}$. Finally, we discuss the implications of the observed chemistry for the structure of the gas and dust in the ISM.

Highlighted results include the following. First, whereas gas in the disk has a molecular hydrogen fraction of 0.65 , clouds on the outer edge of the Galactic bar and in or near the Galactic center have molecular fractions of 0.85 and $>0.9$, respectively. Second, we observe trends in isotope ratios with Galactocentric distance; while carbon and silicon show enhancement of the rare isotopes at low Galactocentric distances, sulfur exhibits no trend with Galactocentric distance. We also determine that the ratio of $c$ - $\mathrm{C}_{3} \mathrm{H}_{2} / c$ $\mathrm{H}^{13} \mathrm{CCCH}$ provides a good estimate of the ${ }^{12} \mathrm{C} /{ }^{13} \mathrm{C}$ ratio, whereas $\mathrm{H}_{2} \mathrm{CO} / \mathrm{H}_{2}^{13} \mathrm{CO}$ exhibits fractionation. Third, we report the presence of $l-\mathrm{C}_{3} \mathrm{H}^{+}$in diffuse clouds for the first time. Finally, we suggest that $\mathrm{CS}$ has an enhanced abundance within higher density clumps of material in the disk, and therefore may be diagnostic of cloud conditions. If this holds, the diffuse clouds in the Galactic disk contain multiple embedded hyperdensities in a clumpy structure, and the density profile is not a simple function of $A_{\mathrm{V}}$.
\end{abstract}

Key words. astrochemistry - radio lines: ISM - ISM: abundances - molecular processes - ISM: structure - Galaxy: center

\section{Introduction}

The diffuse ISM serves as a significant reservoir for gas in the Galaxy, and is believed to be involved in processes of gas accretion, recycling and feedback from star formation, and in the first stages of molecular cloud formation. Thus, an understanding of this material is essential for understanding the processing of gas in galaxies. Although the first interstellar molecular detections were made in diffuse gas, namely $\mathrm{CH}, \mathrm{CH}^{+}$, and CN (Swings \& Rosenfeld 1937; McKellar 1940; Douglas \& Herzberg 1941), the diffuse ISM was not expected to have a very rich molecular chemistry, and recent observations have proven surprising.

While our picture of the physical and chemical structure of the diffuse ISM continues to evolve, the chemistry reveals three primary phases with blurred boundaries. First, the most diffuse material produces absorption primarily by certain light hydride species (e.g., Gerin et al. 2010a; Qin et al. 2010; Indriolo et al. 2015). The absorption components tend to be broad $\Delta v \gtrsim 15 \mathrm{~km} \mathrm{~s}^{-1}$, indicating large

$\star$ The reduced spectra (FITS files) are only available at the CDS via anonymous ftp to cdsarc.u-strasbg. fr (130.79.128.5) or via http://cdsarc.u-strasbg.fr/viz-bin/qcat?J/A+A/610/A10 spatial extents, and estimated physical conditions include low densities $\left(50<n<300 \mathrm{~cm}^{-3}\right)$, warm kinetic temperatures $(80<T<300 \mathrm{~K})$ (Snow \& McCall 2006), and low molecular hydrogen fractions $\left(f\left(\mathrm{H}_{2}\right)=\frac{2 N_{\mathrm{H}_{2}}}{N_{\mathrm{HI}}+2 N_{\mathrm{H}_{2}}}<0.1\right)$ (Indriolo et al. 2015; Snow \& McCall 2006). In the second phase, heavier molecules appear, including $\mathrm{HCN}, \mathrm{CS}, \mathrm{H}_{2} \mathrm{CO}$, and $c-\mathrm{C}_{3} \mathrm{H}_{2}$, but estimated physical conditions still include low densities $\left(100<n<500 \mathrm{~cm}^{-3}\right)$ and warm temperatures (Snow \& McCall 2006; Liszt et al. 2006, 2012). In this phase, higher molecular fractions are present, with $f\left(\mathrm{H}_{2}\right) \approx 0.4$ (Liszt et al. 2012), and extinction conditions of $A_{\mathrm{V} 0} \gtrsim 0.2 \mathrm{mag}$ (implying a total extinction $A_{\mathrm{V}} \gtrsim 0.4$ ) are typical (Snow \& McCall 2006).

The third phase is a translucent cloud, which is historically defined as material with a central extinction of $1<A_{\mathrm{V} 0}<2.5$ and more recently defined as a cloud where $f\left(\mathrm{C}^{+}\right)<0.5$ and $f(\mathrm{CO})<0.9$, so that carbon is present in neutral, ionized, and molecular forms concurrently (Snow \& McCall 2006). Translucent clouds are embedded within diffuse clouds, and their kinematic signatures include absorption over the same general velocity range as the diffuse material, but with narrower features. Galactic disk translucent clouds have densities of order $5000 \mathrm{~cm}^{-3}$ and cool temperatures of $15<T<30 \mathrm{~K}$. In Galactic center translucent clouds, densities may be higher, up to 
$10^{4} \mathrm{~cm}^{-3}$ (Greaves et al. 1992; Greaves \& Williams 1994), and temperatures are much warmer at $50<T<90 \mathrm{~K}$.

In all three phases, standard chemical models underpredict the molecular abundances of most observed molecules by multiple orders of magnitude (Lucas \& Liszt 2002; Godard et al. 2012), indicating a poor understanding of the physics and chemistry of these regions. Models incorporating turbulent dissipation, where material is heated and compressed periodically, produce model predictions that are more consistent with observations for light hydride species in diffuse clouds (Godard et al. $2009,2012)$. Yet much work remains to be done in order to understand the coupled physics and chemistry within the diffuse and translucent media, including the physical and chemical exchange between the three phases.

Furthermore, the diffuse and translucent gas in the Galactic center appears to be systematically different from that observed in the disk by multiple measures. In addition to the temperature and density differences noted above, observations reveal very high abundances of $\mathrm{H}_{3}^{+}$with a filling factor of nearly 1 (Oka et al. 2005) and a larger fractional volume filled by translucent clouds in the Galactic center compared to the disk, due largely to the extreme amount of gas mass in the Galactic center (Morris \& Serabyn 1996). Cosmic ray (CR) ionization rates in diffuse gas in the Galactic center have been measured to be enhanced by 1 to 3 orders of magnitude compared to the disk (Le Petit et al. 2016; Indriolo et al. 2015), and significantly higher X-ray fluxes are also present (Amo-Baladrón et al. 2009). It is not precisely known how this affects the chemistry in these regions; several small molecules including $\mathrm{H}_{3}^{+}, \mathrm{OH}^{+}$, and $\mathrm{H}_{2} \mathrm{O}^{+}$ are predicted and observed to have enhanced abundances in high CR flux environments, but for most larger neutral species, chemical models predict lower abundances due to molecular dissociation (e.g., Quan et al. 2016).

In this paper, we present absorption profiles by 11 molecules and five isotopologues in the line-of-sight to Sgr B2(N), as measured by the GBT PRebiotic MOlecular Survey (PRIMOS). PRIMOS is the most sensitive broadband survey at centimeter wavelengths, conducted toward Sgr B2(N), a high-mass star forming region in the Galactic center known for its extreme mass and diversity of molecular chemistry. In the $1-50 \mathrm{GHz}$ spectrum obtained by PRIMOS, foreground absorption is detected from $\sim 50$ molecular transitions of 17 molecules and six isotopologues. In this work, we present the kinematic structure and abundance patterns of the sulfur-bearing species CS, SO, $\mathrm{CCS}, \mathrm{H}_{2} \mathrm{CS}$, and $\mathrm{HCS}^{+}$; oxygen-bearing molecules $\mathrm{OH}, \mathrm{SiO}$, and $\mathrm{H}_{2} \mathrm{CO}$; simple hydrocarbon molecules $c-\mathrm{C}_{3} \mathrm{H}_{2}, l-\mathrm{C}_{3} \mathrm{H}$, and $l-\mathrm{C}_{3} \mathrm{H}^{+}$; and detected isotopologues of these species. We exclude from this paper detected line-of-sight absorption by $\mathrm{NH}_{3}$, as absorption in the metastable $\mathrm{NH}_{3}$ transitions should be treated in a different analysis, and by complex organic molecules (COMs), which will be presented in a second paper (Paper II; Corby et al., in prep.) to closely follow.

While absorption and emission associated with Sgr B2 spans the velocity range of +40 to $+90 \mathrm{~km} \mathrm{~s}^{-1}$, diffuse and translucent clouds believed to be located in the Galactic center, bar, and spiral arms are observed in the range of -130 to $+40 \mathrm{~km} \mathrm{~s}^{-1}$. While the exact locations of most of the cloud components are not confidently known, the following guidelines are helpful for interpreting abundance patterns. Features in the velocity range of -130 to $-55 \mathrm{~km} \mathrm{~s}^{-1}$ are believed to be located within $1 \mathrm{kpc}$ of the Galactic center, and although some of the material at $0 \mathrm{~km} \mathrm{~s}^{-1}$ is presumably local to the Orion Arm, most of the absorption from -10 to $+25 \mathrm{~km} \mathrm{~s}^{-1}$ also originates from within or near the Galactic center. Amongst the most prominent features, the
$-106 \mathrm{~km} \mathrm{~s}^{-1}$ cloud is associated with the Expanding Molecular Ring (EMR) that spans the Galactic center and marks the outer edge of the CMZ at a 200 to $300 \mathrm{pc}$ galactocentric distance (Whiteoak \& Gardner 1979); the $-73 \mathrm{~km} \mathrm{~s}^{-1}$ cloud is suggested to be part of the 1-kpc disk located deep within the Galactic bar (Wirström et al. 2010); and while some researchers have suggested that the $\sim 0 \mathrm{~km} \mathrm{~s}^{-1}$ component consists of ejecta from Sgr B2 (Wirström et al. 2010), multiple observations have shown that this feature is widespread across the Galactic center, and is a rather unique system containing molecular line absorption and weak recombination line emission across the Galactic center (Jones et al. 2012; Royster \& Yusef-Zadeh 2014), as well as weak masing in the viscinity of Sgr B2(N) (Corby et al. 2015). The gas from -55 to $-35 \mathrm{~km} \mathrm{~s}^{-1}$ is associated with gas in the near 3-kpc arm located on the outer edge of the Galactic bar, while the material from -35 to $-10 \mathrm{~km} \mathrm{~s}^{-1}$ is believed to arise in a spiral arm in the disk at a Galactocentric distance of $\sim 4 \mathrm{kpc}$ (Whiteoak \& Gardner 1979; Wirström et al. 2010).

The remainder of this paper is organized as follows. In Sect. 2, we describe the observations. In Sect. 3, we present the line profiles (Sect. 3.1), cloud kinematics (Sect. 3.2), and measured column densities and abundances (Sects. 3.3 and 3.5). In Sect. 4, we discuss the method for molecular hydrogen column estimation. In Sect. 5, we discuss the results in the context of trends with Galactocentric distance and implications for the cloud structures, before concluding in Sect. 6.

The densities in diffuse and translucent clouds are insufficient to excite molecules much beyond the CMB temperature, and excitation temperatures within this material are observed to span the range of $2.7 \mathrm{~K} \lesssim T_{\text {ex }} \lesssim 4 \mathrm{~K}$ (Greaves \& Williams 1994; Linke et al. 1981). As a result, the equation for line optical depth reduces to

$\tau=-\ln \left(1+T_{\mathrm{L}} / T_{\mathrm{C}}\right)$

for baseline subtracted absorption strength $T_{\mathrm{L}}$ absorbing against a continuum source of strength $T_{\mathrm{C}}$. The value $T_{\mathrm{L}} / T_{\mathrm{C}}$ fully characterizes the line optical depth, and the data are therefore presented in this scale. The scale additionally provides higher precision measurements, as many sources of calibration error that typically apply to single dish radio data cancel in this ratio, including atmospheric opacity corrections, telescope efficiencies, and absolute flux calibrations.

\section{Observations}

The observations were conducted with the Robert C. Byrd Green Bank Telescope (GBT) through the PRIMOS ${ }^{1}$. PRIMOS is a key science project of the GBT that provides the deepest, most frequency-complete centimeter-wave spectral survey completed to date. For all observations, the telescope was pointed toward the position of the Large Molecule Heimat (LMH) at $\alpha=$ $17^{\mathrm{h}} 47^{\mathrm{m}} 19.8, \delta=-28^{\circ} 22^{\prime} 17^{\prime \prime}$. The LMH is a hot core source of 5 arcsec in spatial extent located on the SW edge of the Sgr $\mathrm{B} 2(\mathrm{~N})$ continuum structure. The GBT beam, which varies from 13 arcmin at $1 \mathrm{GHz}$ to $15 \operatorname{arcsec}$ at $50 \mathrm{GHz}$, is sensitive to absorption in the foreground of the free-free continuum structure of Sgr B2(N) as well as molecular line emission from the LMH over the full range of frequencies. At very low frequencies (of $<8 \mathrm{GHz}$ ), the beam contains Sgr B2(M) as well. Most of the data were collected over the full year of 2007 , with some observations performed in 2002, 2005, and 2013. All observations were

\footnotetext{
PRIMOS is publicly available at http://www. cv.nrao.edu/PRIMOS/
} 
Table 1. Summary of molecular lines observed in line-of-sight absorption.

\begin{tabular}{llcccc}
\hline \hline Molecule & Transition & $\begin{array}{c}\text { Frequency } \\
(\mathrm{MHz})\end{array}$ & $\begin{array}{c}E_{\mathrm{L}} \\
(\mathrm{K})\end{array}$ & $\theta_{B}$ & Catalog \\
\hline $\mathrm{OH}$ & $J=\frac{3}{2} \Omega=\frac{3}{2} F=1^{+}-2^{-}$ & $1612.2310(2)$ & 0.0026 & $7.8^{\prime}$ & $\mathrm{JPL}$ \\
$\mathrm{OH}$ & $J=\frac{3}{2} \Omega=\frac{3}{2} F=1^{+}-1^{-}$ & $1665.4018(2)$ & 0.000 & $7.6^{\prime}$ & JPL \\
$\mathrm{OH}$ & $J=\frac{3}{2} \Omega=\frac{3}{2} F=2^{+}-2^{-}$ & $1667.3590(2)$ & 0.0026 & $7.6^{\prime}$ & JPL \\
$c-\mathrm{C}_{3} \mathrm{H}_{2}$ & $1_{10}-1_{01}$ & $18343.143(2)$ & 2.35 & $41.2^{\prime \prime}$ & $\mathrm{CDMS}$ \\
$c-\mathrm{C}_{3} \mathrm{H}_{2}$ & $2_{20}-2_{11}$ & $21587.4008(3)$ & 8.67 & $35.0^{\prime \prime}$ & JPL \\
$c-\mathrm{C}_{3} \mathrm{H}_{2}$ & $2_{11}-2_{02}$ & $46755.610(2)$ & 6.43 & $16.2^{\prime \prime}$ & $\mathrm{CDMS}$ \\
$c-\mathrm{H}^{13} \mathrm{CCCH}$ & $1_{10}-1_{01}$ & $18413.8248(7)$ & 2.29 & $41.1^{\prime \prime}$ & $\mathrm{CDMS}$ \\
$\mathrm{H}_{2} \mathrm{CO}$ & $1_{10}-1_{11}$ & $4829.660(1)$ & 15.16 & $2.6^{\prime}$ & JPL \\
$\mathrm{H}_{2} \mathrm{CO}$ & $2_{11}-2_{12}$ & $14488.479(1)$ & 21.92 & $52.2^{\prime \prime}$ & JPL \\
$\mathrm{H}_{2} \mathrm{CO}$ & $3_{12}-3_{13}$ & $28974.805(10)$ & 32.060 & $26.1^{\prime \prime}$ & JPL \\
$\mathrm{H}_{2}^{13} \mathrm{CO}$ & $1_{10}-1_{11}$ & $4593.0885(1)$ & 15.126 & $2.7^{\prime \prime}$ & JPL \\
$\mathrm{H}_{2}^{13} \mathrm{CO}$ & $2_{11}-2_{12}$ & $13778.8041(2)$ & 21.723 & $54.9^{\prime \prime}$ & JPL \\
${ }^{28} \mathrm{SiO}$ & $1-0$ & $43423.76(5)$ & 0.000 & $17.4^{\prime \prime}$ & $\mathrm{CDMS}$ \\
${ }^{29} \mathrm{SiO}$ & $1-0$ & $42879.949(3)$ & 0.000 & $17.6^{\prime \prime}$ & $\mathrm{CDMS}$ \\
${ }^{30} \mathrm{SiO}$ & $1-0$ & $42373.427(5)$ & 0.000 & $17.8^{\prime \prime}$ & $\mathrm{CDMS}$ \\
$\mathrm{SO}$ & $1_{0}-0_{1}$ & $30001.58(10)$ & 0.000 & $25.2^{\prime \prime}$ & $\mathrm{CDMS}$ \\
$\mathrm{CS}$ & $1-0$ & $48990.955(2)$ & 0.000 & $15.4^{\prime \prime}$ & $\mathrm{CDMS}$ \\
$\mathrm{C}^{34} \mathrm{~S}$ & $1-0$ & $48206.941(2)$ & 0.000 & $15.7^{\prime \prime}$ & $\mathrm{CDMS}$ \\
$\mathrm{CCS}$ & $N_{J}=1_{2}-2_{1}$ & $22344.031(1)$ & 0.533 & $33.8^{\prime \prime}$ & $\mathrm{CDMS}$ \\
$\mathrm{CCS}$ & $N_{J}=2_{3}-1_{2}$ & $33751.370(1)$ & 1.606 & $22.4^{\prime \prime}$ & $\mathrm{CDMS}$ \\
$\mathrm{HCS}$ & $1-0$ & $42674.195(1)$ & 0.000 & $17.7^{\prime \prime}$ & $\mathrm{CDMS}$ \\
$\mathrm{H}_{2} \mathrm{CS}$ & $1_{01}-0_{00}$ & $34351.43(2)$ & 0.000 & $22.0^{\prime \prime}$ & $\mathrm{CDMS}$ \\
$l-\mathrm{C}_{3} \mathrm{H}$ & $J=\frac{3}{2}-\frac{1}{2} \Omega=\frac{1}{2} F=2-1 \mathrm{f}$ & $32627.297(2)$ & 0.00086 & $23.2^{\prime \prime}$ & $\mathrm{CDMS}$ \\
$l-\mathrm{C}_{3} \mathrm{H}$ & $J=\frac{3}{2}-\frac{1}{2} \Omega=\frac{1}{2} F=2-1 \mathrm{e}$ & $32660.645(2)$ & 0.00086 & $23.1^{\prime \prime}$ & $\mathrm{CDMS}$ \\
$l-\mathrm{C}_{3} \mathrm{H}^{+}$ & $1-0$ & $22489.864(2)$ & 0.00 & $33.6^{\prime \prime}$ & $\mathrm{CDMS}$ \\
$l-\mathrm{C}_{3} \mathrm{H}^{+}$ & $2-1$ & $44979.544(3)$ & 1.079 & $16.8^{\prime \prime}$ & $\mathrm{CDMS}$ \\
\hline & & & &
\end{tabular}

performed in position switching mode, with an OFF position located 1 degree $\mathrm{E}$ in azimuth. Over most of the frequency range (of $>3 \mathrm{GHz}$ ), four spectral windows of $200 \mathrm{MHz}$ bandwidth and 8192 channels were observed simultaneously; $50 \mathrm{MHz}$ windows were observed at frequencies of $<3 \mathrm{GHz}$. Most windows were observed for $\sim 10-15 \mathrm{~h}$, resulting in $T_{\mathrm{A}}^{*} \sim 5 \mathrm{mK}$ rms noise levels in most of the frequency range.

The absorption profiles are carefully baseline subtracted over the narrow frequency ranges corresponding to about -400 to $+250 \mathrm{~km} \mathrm{~s}^{-1}$ using a polynomial solution. For data with very stable baselines (typically $Q$ band), we used a first order baseline. For data with more highly varying baselines, we usually used a fourth order polynomial fit over a typical velocity range of -400 to $+250 \mathrm{~km} \mathrm{~s}^{-1}$. The data were then normalized by the continuum, as the ratio of $T_{\mathrm{L}} / T_{\mathrm{C}}$ is the fundamental value for characterizing the molecular line absorption from the diffuse and translucent clouds. This ratio is independent of corrections to the atmospheric attenuation and the GBT aperture efficiency, and these corrections therefore were not applied.

\section{Results}

In observations toward Sgr B2(N), two absorption components are associated with Sgr B2 itself, at +64 and $+82 \mathrm{~km} \mathrm{~s}^{-1}$ (Huettemeister et al. 1995; Corby et al. 2015). We assume that line absorption and emission in the range of +40 to $+90 \mathrm{~km} \mathrm{~s}^{-1}$ is from Sgr B2 and refrain from further discussing this material, instead focusing on the molecular composition of clouds observed in the velocity range of -126 to $+24 \mathrm{~km} \mathrm{~s}^{-1}$, associated with material in the line of sight to Sgr B2. Throughout this section, we assume that excitation conditions in all absorbing components are equivalent and characterized by $T_{\mathrm{ex}}=3 \mathrm{~K}$. This assumption has been validated in the line of sight to Sgr B2(M) for CS (Greaves \& Williams 1994), and excitation temperatures of 2.73 to $3 \mathrm{~K}$ have been widely adopted for studies of diffuse and translucent clouds in line-of-sight absorption (e.g., Nyman 1984; Greaves \& Nyman 1996; Lucas \& Liszt 1993; Qin et al. 2010; Liszt et al. 2012; Wiesemeyer et al. 2016). The assumption is further validated in Sect. 3.3, in which we explore the line excitation of molecules sampled by multiple transitions in the PRIMOS data. By assuming equivalent excitation in all line-ofsight clouds, it is possible to directly compare column densities in different clouds from a single line profile.

\subsection{Spectral profiles}

Table 1 lists transitions that were observed in line-of-sight absorption with a sufficient signal-to-noise ratio $(\mathrm{S} / \mathrm{N})$ and adequate baseline stability to enable a fair characterization. For all listed transitions, we fit Gaussian components over the velocity range of -126 to $+24 \mathrm{~km} \mathrm{~s}^{-1}$ using the FITGAUSS function provided in the GBTIDL data reduction package and/or with a Gaussian fitting function constructed by the authors. We attempted to use as few Gaussian components as could account for the line profile to within the noise level and we determined errors to the fits as the square root of the diagonal entries of the covariance matrix. Gaussian fits are overlaid on all spectral profiles shown, and individual Gaussian components are shown with a dashed profile. For transitions with resolvable hyperfine structure or A/E splitting including lines of $l-\mathrm{C}_{3} \mathrm{H}\left(J=\frac{3}{2}-\frac{1}{2}\right.$, $\Omega=\frac{1}{2}$ ), we fit the primary hyperfine component and assumed that the satellite lines have the same line shape with the height set by the relative line strengths of the satellite and primary features. Selected line profiles are shown throughout this subsection, and the full catalog of line profiles listed in Table 1 are shown in Appendix A. 

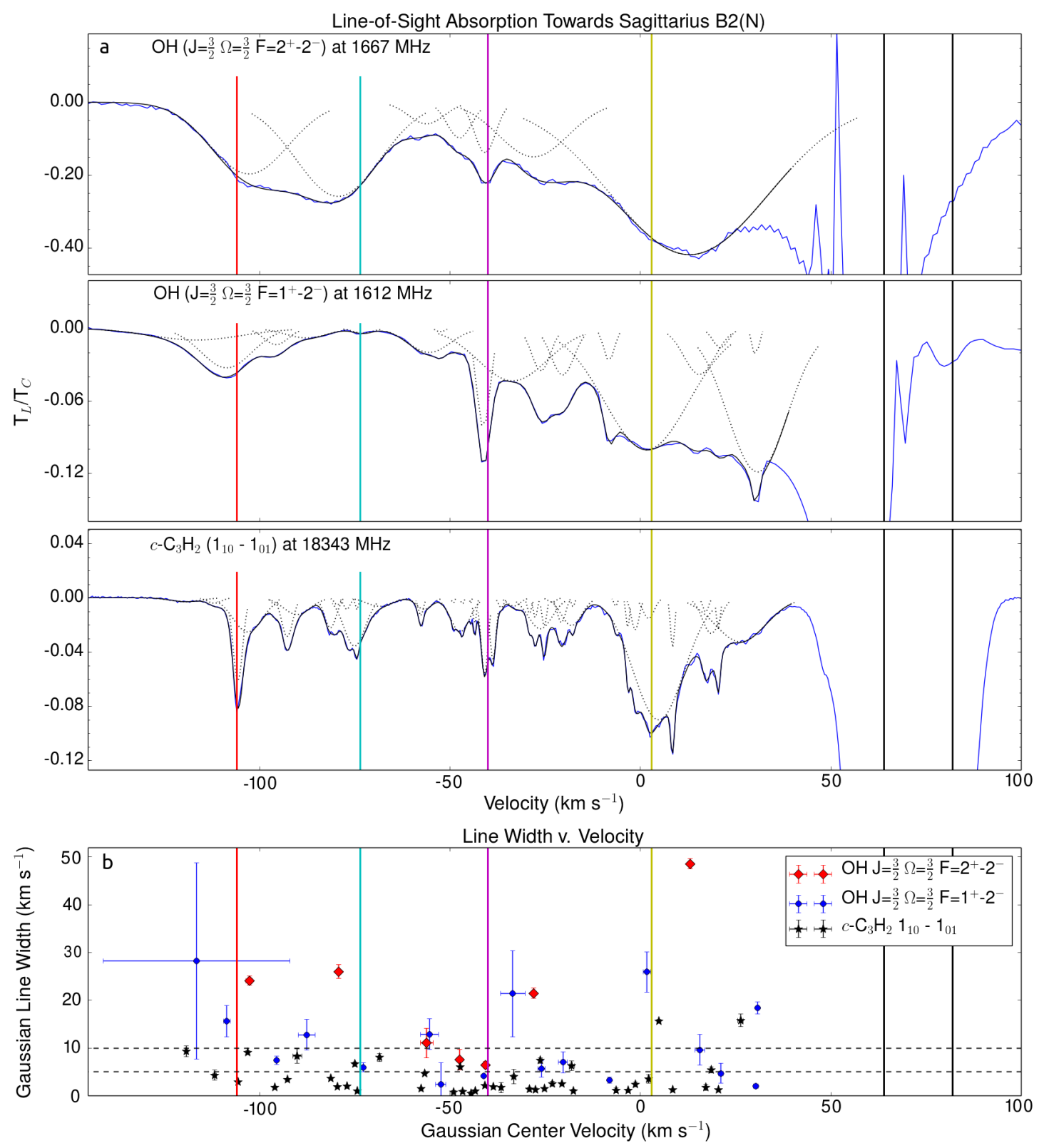

Fig. 1. a) Line-of-sight profiles of $\mathrm{OH}$ and $c-\mathrm{C}_{3} \mathrm{H}_{2}$ are plotted in blue with individual Gaussian fit components shown with black dashed lines and the complete Gaussian fit overlaid in solid black. $b$ ) Gaussian fit parameters of center velocity and line width for individual Gaussian components fit to the profiles of $\mathrm{OH}$ and $c-\mathrm{C}_{3} \mathrm{H}_{2}$. Horizontal dashed lines indicate line widths of $5 \mathrm{~km} \mathrm{~s}^{-1}$ and $10 \mathrm{~km} \mathrm{~s}^{-1}$. In all panels, black vertical lines indicate the velocities of line absorption by Sgr B2 at +64 and $+82 \mathrm{~km} \mathrm{~s}^{-1}$, and colored lines are located at $-106,-73.5,-40$, and $+3 \mathrm{~km} \mathrm{~s}{ }^{-1}$.

\subsection{1. $\mathrm{OH}$ and $c-\mathrm{C}_{3} \mathrm{H}_{2}$}

Figure 1 shows the absorption profiles and Gaussian component fits to selected lines of $\mathrm{OH}$ and $c-\mathrm{C}_{3} \mathrm{H}_{2}$. The profile of $\mathrm{OH}\left(J=\frac{3}{2} \Omega=\frac{3}{2} F=2^{+}-2^{-}\right)$at $1667 \mathrm{MHz}$ includes very broad line components, many of which have $\Delta v \gtrsim 20 \mathrm{~km} \mathrm{~s}^{-1}$, and the absorption fully covers the velocity range of -120 to $+40 \mathrm{~km} \mathrm{~s}^{-1}$ with no absorption-free channels. While the $\mathrm{OH}$ $\left(J=\frac{3}{2} \Omega=\frac{3}{2} F=1^{+}-1^{-}\right)$transition at $1665 \mathrm{MHz}$ is extremely similar to the profile of the $1667 \mathrm{MHz}$ transition (Appendix A), the $\left(J=\frac{3}{2} \Omega=\frac{3}{2} F=1^{+}-2^{-}\right)$line profile at $1612 \mathrm{MHz}$ appears somewhat different. The $1612 \mathrm{MHz}$ transition has deeper absorption by the $-40 \mathrm{~km} \mathrm{~s}^{-1}$ cloud and weaker absorption in the velocity range of -90 to $-60 \mathrm{~km} \mathrm{~s}^{-1}$, as discussed in Sect. 3.5.1. While the line absorption at $1612 \mathrm{MHz}$ also fully covers the velocity range of -120 to $+40 \mathrm{~km} \mathrm{~s}^{-1}$ and contains broad $\left(\Delta v \gtrsim 10 \mathrm{~km} \mathrm{~s}^{-1}\right.$ ) Gaussian components over this range, lower line width features, of $3 \lesssim \Delta v \lesssim 10 \mathrm{~km} \mathrm{~s}^{-1}$, are superimposed on the broad line absorption. In contrast to the broad line widths of the $1667 \mathrm{MHz} \mathrm{OH}$ transition in particular, absorption by $c-\mathrm{C}_{3} \mathrm{H}_{2}$ consists of comparatively narrow features, leaving segments of the spectrum without detectable absorption. In this profile, many narrow components (with $\Delta v \lesssim 3 \mathrm{~km} \mathrm{~s}^{-1}$ ) are superimposed on moderately broad components with $3 \lesssim \Delta v \lesssim 10 \mathrm{~km} \mathrm{~s}^{-1}$.

In the line absorption by $c-\mathrm{C}_{3} \mathrm{H}_{2}$, at least ten line-of-sight absorption components are clearly distinguishable, peaking at velocities from -106 to $+25 \mathrm{~km} \mathrm{~s}^{-1}$. Throughout this section, we treat these features independently and refer to these clouds by their approximate center velocities. The center velocities and velocity ranges considered are provided in Table 2 . Due to the nearly constant abundance of $c-\mathrm{C}_{3} \mathrm{H}_{2}$ in diffuse and translucent 


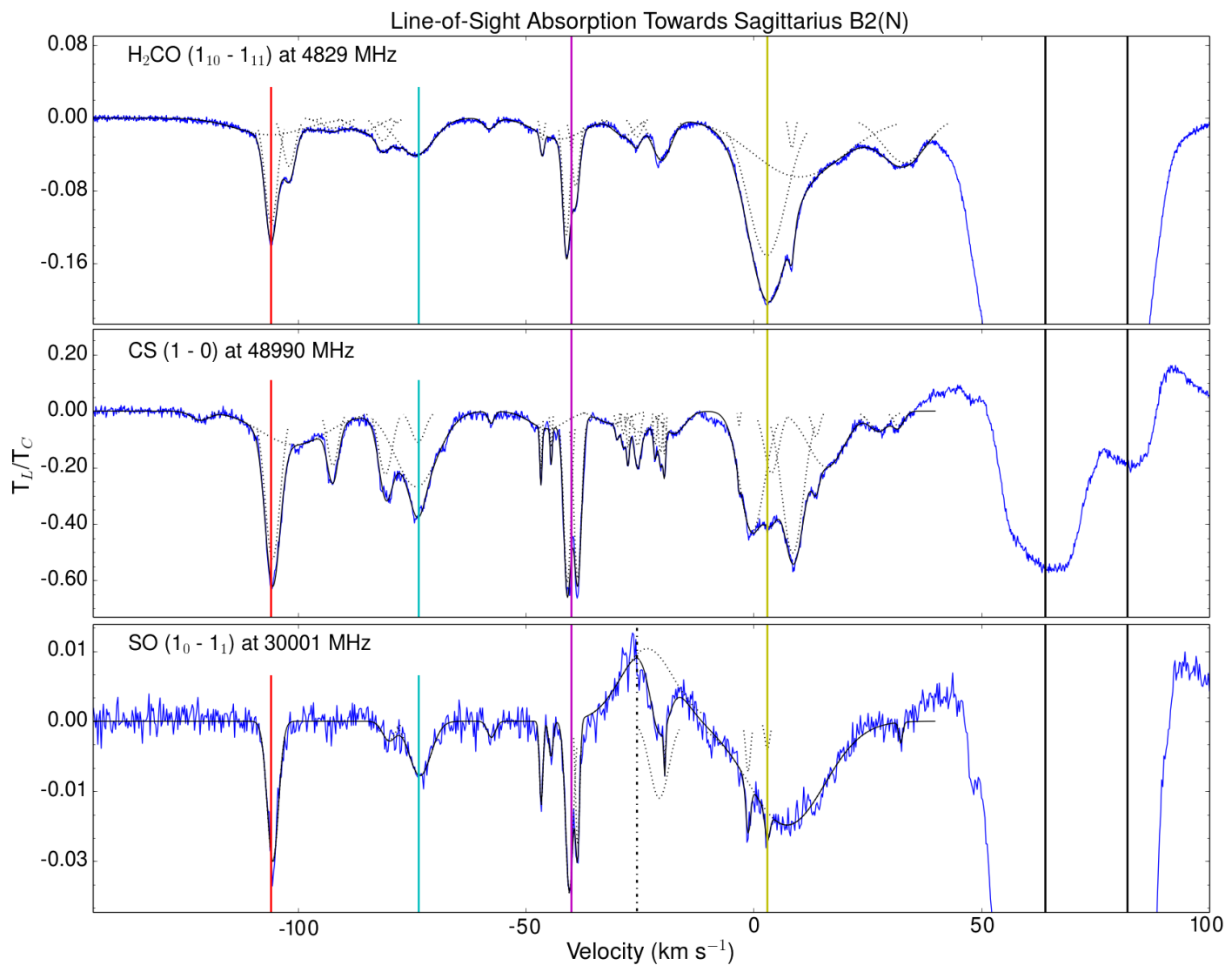

Fig. 2. Absorption profiles of $\mathrm{H}_{2} \mathrm{CO}, \mathrm{CS}$, and $\mathrm{SO}$ are overlaid by individual best fit Gaussian components (black dotted line) and the sum of best-fit Gaussians (black solid line). Black vertical lines indicate the velocities of line absorption by Sgr B2 at +64 and $+82 \mathrm{~km} \mathrm{~s}^{-1}$, and colored lines are located at $-106,-73.5,-40$, and $+3 \mathrm{~km} \mathrm{~s}^{-1}$. In the profile of SO, the black dashed-dotted line indicates the $8_{17}^{-}-8_{18}^{+}$transition of $\mathrm{CH}_{3} \mathrm{OH}$ at $+64 \mathrm{~km} \mathrm{~s}^{-1}$, which appears in emission.

Table 2. Velocity ranges considered for each cloud.

\begin{tabular}{lrr}
\hline \hline $\begin{array}{l}\text { Nominal velocity } \\
\left(\mathrm{km} \mathrm{s}^{-1}\right)\end{array}$ & $\begin{array}{c}v_{0} \\
\left(\mathrm{~km} \mathrm{~s}^{-1}\right)\end{array}$ & $\begin{array}{c}v_{1} \\
\left(\mathrm{~km} \mathrm{~s}^{-1}\right)\end{array}$ \\
\hline-120 & -126 & -116 \\
-106 & -110 & -97 \\
-92 & -97 & -86 \\
-80 & -86 & -78 \\
-73 & -78 & -62 \\
-58 & -62 & -53 \\
-47 & -53 & -42.5 \\
-40 & -42.5 & -35 \\
-23 & -35 & -12 \\
0 & -12 & 15 \\
20 & 15 & 24 \\
\hline
\end{tabular}

gas (Liszt et al. 2012), we use this species as a diagnostic for the total column of $\mathrm{H}_{2}$ and compare the profiles of other species to $c-\mathrm{C}_{3} \mathrm{H}_{2}$. We further discuss the validity of this in Sect. 4.1.

\subsection{2. $\mathrm{H}_{2} \mathrm{CO}$, CS, and SO}

Figure 2 shows high $\mathrm{S} / \mathrm{N}$ transitions of $\mathrm{H}_{2} \mathrm{CO}, \mathrm{CS}$, and SO. $\mathrm{H}_{2} \mathrm{CO}$ and $\mathrm{CS}$ are detected in the same ten velocity components as $c-\mathrm{C}_{3} \mathrm{H}_{2}$, while $\mathrm{SO}$ is detected in all components with the exception of the $-92 \mathrm{~km} \mathrm{~s}^{-1}$ cloud. Line absorption by $c-\mathrm{C}_{3} \mathrm{H}_{2}$ and $\mathrm{H}_{2} \mathrm{CO}$ are fairly similar but have notable differences. Whereas absorption by $c-\mathrm{C}_{3} \mathrm{H}_{2}$ is of a similar peak absorption strength in the $-92,-73,-40$, and $-23 \mathrm{~km} \mathrm{~s}^{-1}$ clouds, the $\mathrm{H}_{2} \mathrm{CO}$ absorption varies more substantially in these components. In the $\mathrm{H}_{2} \mathrm{CO}$ profile, the $-73 \mathrm{~km} \mathrm{~s}^{-1}$ and the $-23 \mathrm{~km} \mathrm{~s}^{-1}$ components have similar peak absorption strengths, whereas the $-40 \mathrm{~km} \mathrm{~s}^{-1}$ component is a factor of $\sim 3$ stronger and the $-92 \mathrm{~km} \mathrm{~s}^{-1}$ cloud is significantly weaker. Additionally, whereas peak absorption by $c-\mathrm{C}_{3} \mathrm{H}_{2}$ is $\sim 40$ percent stronger at $-106 \mathrm{~km} \mathrm{~s}^{-1}$ than in the $-40 \mathrm{~km} \mathrm{~s}^{-1}$ cloud, $\mathrm{H}_{2} \mathrm{CO}$ has deeper absorption in the $-40 \mathrm{~km} \mathrm{~s}^{-1}$ cloud than in the $-106 \mathrm{~km} \mathrm{~s}^{-1}$ cloud.

The absorption profile of CS (1-0) appears qualitatively distinct from the line profiles of $c-\mathrm{C}_{3} \mathrm{H}_{2}$ and $\mathrm{H}_{2} \mathrm{CO}$. Whereas $c-\mathrm{C}_{3} \mathrm{H}_{2}$ and $\mathrm{H}_{2} \mathrm{CO}$ exhibit comparatively smooth absorption profiles, $\mathrm{CS}$ line absorption is characterized by sharper, more jagged features. This is particularly true of the clusters of clouds around $-47,-40$, and $-23 \mathrm{~km} \mathrm{~s}^{-1}$, where CS absorbs in narrow, sharp peaks without as much broad-component absorption as is present in the line profiles of $c-\mathrm{C}_{3} \mathrm{H}_{2}$ and $\mathrm{H}_{2} \mathrm{CO}$. This is best illustrated by the $-47 \mathrm{~km} \mathrm{~s}^{-1}$ gas, in which the absorption depths of the narrow features are significantly greater than the strength of the broad absorption component in the CS profile, whereas the opposite is true for $c-\mathrm{C}_{3} \mathrm{H}_{2}$ and $\mathrm{H}_{2} \mathrm{CO}$. Similarly, absorption by SO $\left(1_{0}-1_{1}\right)$ has narrow lines with little broad-component absorption in the -47 and $-40 \mathrm{~km} \mathrm{~s}^{-1}$ clouds.

\subsubsection{CS, CCS, $\mathrm{HCS}^{+}$, and $\mathrm{H}_{2} \mathrm{CS}$}

Figure 3 includes selected line profiles of CS-bearing molecules. Generally, the profiles of $\mathrm{C}^{34} \mathrm{~S}$ and CCS are similar to one another, with strong line absorption in the $-106,-73,-40$, and 


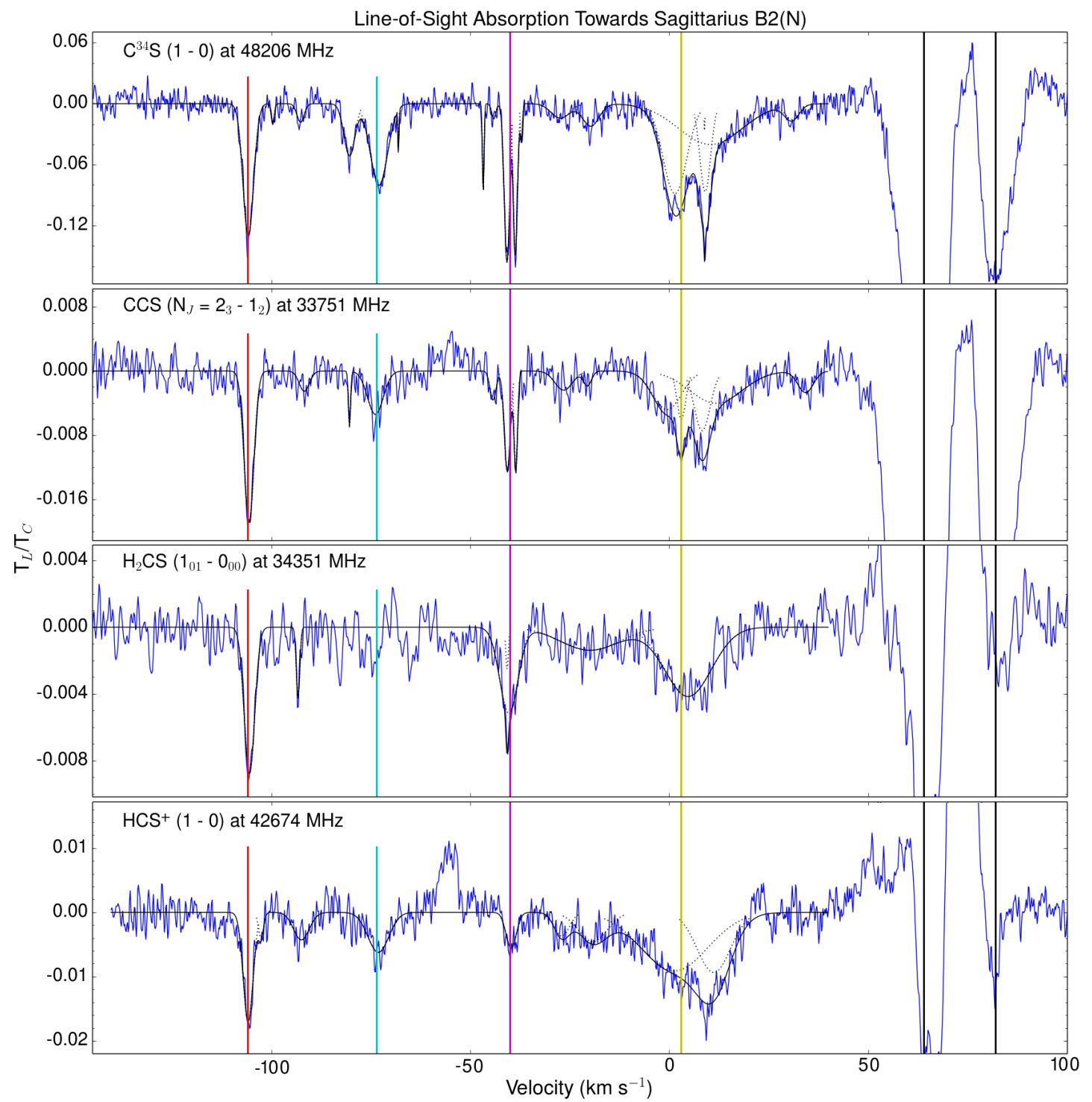

Fig. 3. Absorption profiles of CS-bearing species are overlaid by the best fit Gaussian components (black dotted line) and the sum of best-fit Gaussians (black solid line). Black vertical lines indicate the velocities of line absorption by Sgr B2 at +64 and $+82 \mathrm{~km} \mathrm{~s}^{-1}$, and colored lines are located at $-106,-73.5,-40$, and $+3 \mathrm{~km} \mathrm{~s}^{-1}$.

$0 \mathrm{~km} \mathrm{~s}^{-1}$ clouds and narrow peaks characterizing the -47 and $-40 \mathrm{~km} \mathrm{~s}^{-1}$ gas. The profiles of $\mathrm{H}_{2} \mathrm{CS}$ and $\mathrm{HCS}^{+}$appear to be less characterized by deep, narrow absorption features, although this could be because these two profiles do not have sufficient $\mathrm{S} / \mathrm{N}$ to permit a full characterization of narrow features. The patterns of absorption are somewhat different for these two species than for $\mathrm{CS}, \mathrm{C}^{34} \mathrm{~S}$, and CCS. For $\mathrm{H}_{2} \mathrm{CS}$, little to no absorption is detected at $-73 \mathrm{~km} \mathrm{~s}^{-1}$. In the profile of $\mathrm{HCS}^{+}$, only weak absorption by $\mathrm{HCS}^{+}$is observed in the $-40 \mathrm{~km} \mathrm{~s}^{-1}$ component whereas $\mathrm{CS}, \mathrm{C}^{34} \mathrm{~S}, \mathrm{CCS}$, and $\mathrm{H}_{2} \mathrm{CS}$ produce strong absorption in this cloud.

\subsection{4. $\mathrm{SiO}$}

Figure 4 shows the line profiles of ${ }^{28} \mathrm{SiO}$ and ${ }^{29} \mathrm{SiO}$. Compared to $c-\mathrm{C}_{3} \mathrm{H}_{2}, \mathrm{H}_{2} \mathrm{CO}$, and $\mathrm{CS}$-bearing species especially, the profiles of $\mathrm{SiO}$ isotopologues are much smoother and are predominantly characterized by moderately broad components (with $3 \lesssim \Delta v \lesssim 10 \mathrm{~km} \mathrm{~s}^{-1}$ ). Strong absorption is present particularly at -73 and $0 \mathrm{~km} \mathrm{~s}^{-1}$. Furthermore, the clouds at -73 and
$-80 \mathrm{~km} \mathrm{~s}^{-1}$ do not show any distinction from one another in the $\mathrm{SiO}$ profile; whereas the two clouds have separate peaks in other transitions, they form a single feature in the $\mathrm{SiO}$ profile.

\subsection{5. $l-\mathrm{C}_{3} \mathrm{H}$ and $l-\mathrm{C}_{3} \mathrm{H}^{+}$}

The line profiles of $l-\mathrm{C}_{3} \mathrm{H}$ and $l-\mathrm{C}_{3} \mathrm{H}^{+}$are shown in Fig. 5. As the transitions of $l-\mathrm{C}_{3} \mathrm{H}$ have hyperfine structure, we have only fit the primary $(F=2-1)$ line, with these components shown by dashed Gaussian profiles, and we add the hyperfine structure to the total fit (solid black line) as described above. The presence of the hyperfine structure, combined with the somewhat weak signal and line blending, makes it difficult to characterize the line profiles of $l-\mathrm{C}_{3} \mathrm{H}$. From the $\left(J=\frac{3}{2}-\frac{1}{2} \Omega=\frac{1}{2}\right.$ $F=2-1 l=f$ ) line profile at $32627 \mathrm{MHz}$, we can confidently characterize the absorption at $-106,-92,-80,-73,-40$, -23 , and $0 \mathrm{~km} \mathrm{~s}^{-1}$. In this line profile, we also notice strong absorption at -57 and $-48 \mathrm{~km} \mathrm{~s}^{-1}$, possibly arising from the -58 and $-47 \mathrm{~km} \mathrm{~s}^{-1}$ clouds. In the line-of-sight profiles by other molecules in the PRIMOS data, however, absorption by 
J. F. Corby et al.: The molecular chemistry of diffuse and translucent clouds in the line-of-sight to Sgr B2

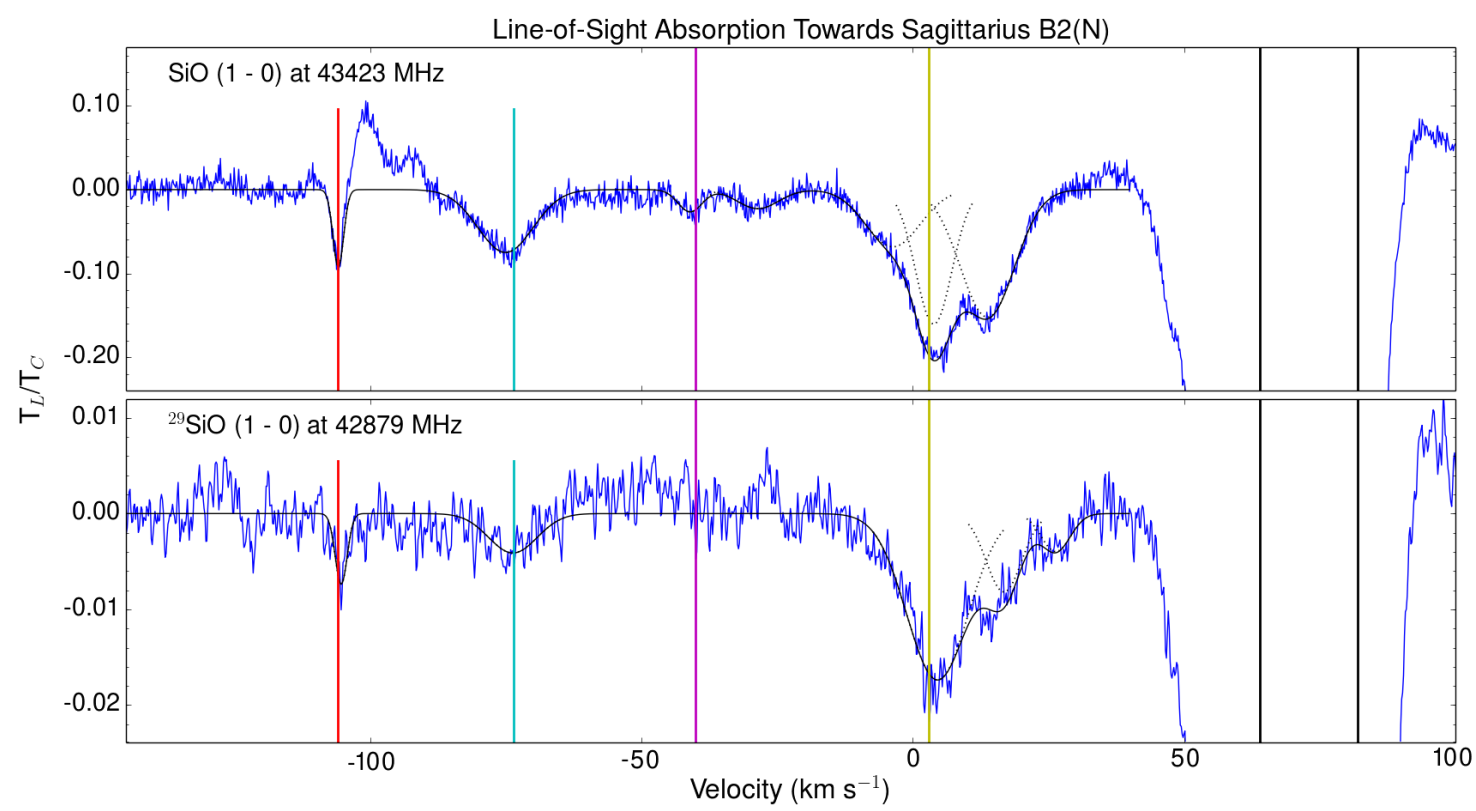

Fig. 4. Absorption profiles of ${ }^{28} \mathrm{SiO}$ and ${ }^{29} \mathrm{SiO}$ are overlaid by the best fit Gaussian components (black dotted line) and the sum of best-fit Gaussians (black solid line). Black vertical lines indicate the velocities of line absorption by Sgr B2 at +64 and $+82 \mathrm{~km} \mathrm{~s}^{-1}$, and colored lines are located at $-106,-73.5,-40$, and $+3 \mathrm{~km} \mathrm{~s}^{-1}$.

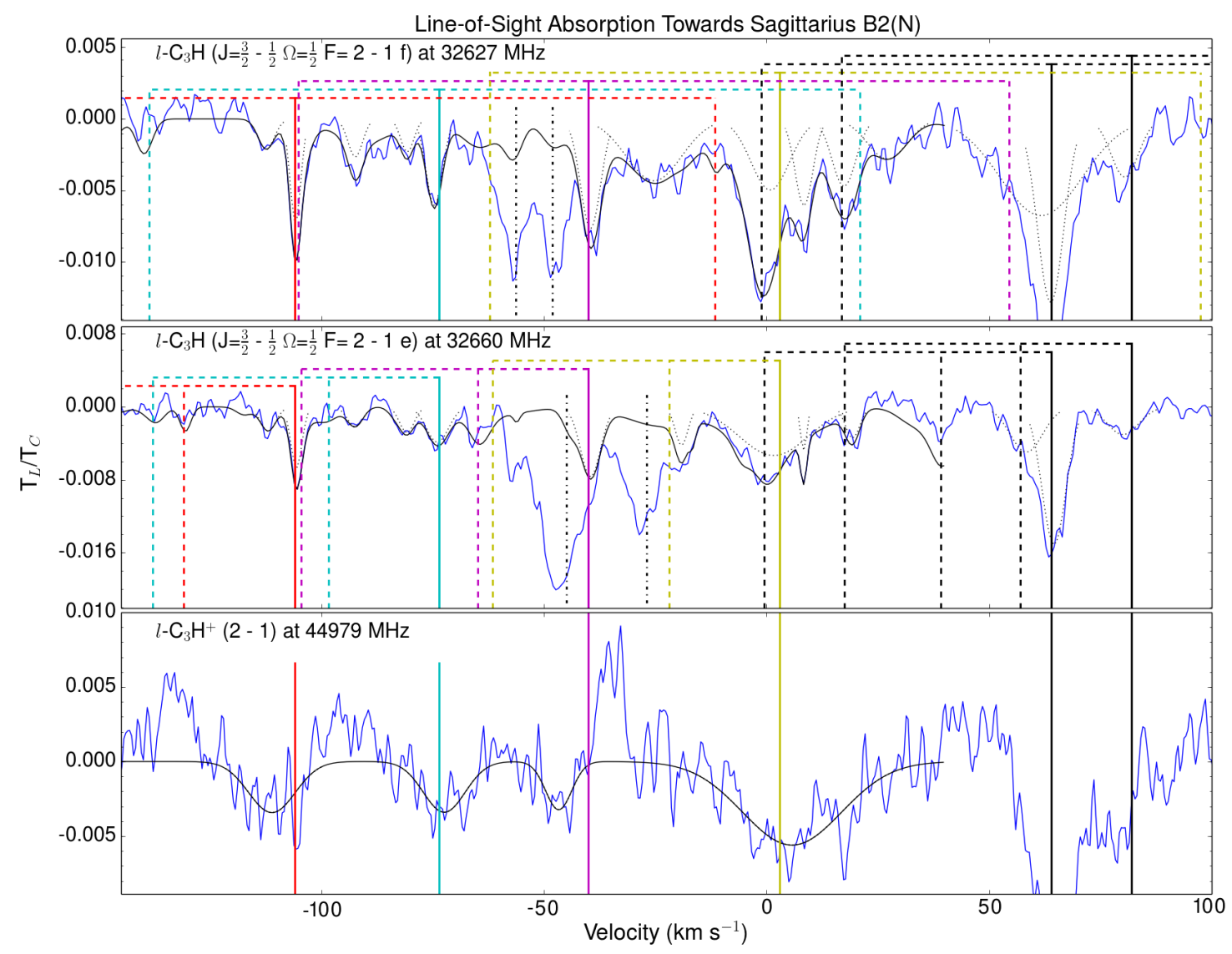

Fig. 5. Absorption profiles of $l-\mathrm{C}_{3} \mathrm{H}$ and $l-\mathrm{C}_{3} \mathrm{H}^{+}$are overlaid by the best fit Gaussian components (black dotted line) and the sum of best-fit Gaussians (black solid line). Black vertical lines indicate the velocities of line absorption by Sgr B2 at +64 and $+82 \mathrm{~km} \mathrm{~s}^{-1}$, and colored lines are located at $-106,-73.5,-40$, and $+3 \mathrm{~km} \mathrm{~s}^{-1}$. Hyperfine structure for each of these velocity components is indicated by dotted vertical lines of the same color. The data are overlaid by Gaussian components fit to the main hyperfine component shown in black dotted lines, and by the total fit to the profile, which assumes that hyperfine or A/E components are present with the same line shape as the primary component, but with the height scaled by the ratio of the line strengths. In the profile of $l-\mathrm{C}_{3} \mathrm{H}$ at $32627 \mathrm{MHz}$, the black dashed-dotted line marks unidentified transitions that are not consistent with the typical profile of diffuse cloud absorption in this line of sight. In the line profile of $l$ - $\mathrm{C}_{3} \mathrm{H}$ at $32660 \mathrm{MHz}$, the black dashed-dotted line marks a transition of $c i s-\mathrm{CH}_{2} \mathrm{OHCHO}$ at velocities of +64 and $+82 \mathrm{~km} \mathrm{~s}^{-1}$ associated with Sgr B2. 
the -58 and $-47 \mathrm{~km} \mathrm{~s}^{-1}$ clouds is weaker than absorption by the -40 and $-73 \mathrm{~km} \mathrm{~s}^{-1}$ clouds. Thus, if the absorption at -57 and $-48 \mathrm{~km} \mathrm{~s}^{-1}$ is from $l-\mathrm{C}_{3} \mathrm{H}$, then the -58 and $-47 \mathrm{~km} \mathrm{~s}^{-1}$ clouds would have exceptionally high abundances of $l-\mathrm{C}_{3} \mathrm{H}$ compared to other clouds. If the features are from $l-\mathrm{C}_{3} \mathrm{H}$, the $(F=1-0)$ hyperfine transitions should occur at -122 and $-113 \mathrm{~km} \mathrm{~s}^{-1}$, respectively, in the rest frame of the $(F=2-1)$ transition. Absorption features are present at both of these velocities, although the observed features are somewhat weaker than the hyperfine lines should be given the strength of the $(F=2-1)$ features. In the $(l=\mathrm{e})$ transition at $32660 \mathrm{MHz}$, the velocity range of -60 to $-20 \mathrm{~km} \mathrm{~s}^{-1}$ is obscured by blending with line absorption from cis-glycolaldehyde (cis- $\mathrm{CH}_{2} \mathrm{OHCHO}$ ) in $\mathrm{Sgr} \mathrm{B} 2$ at +64 and $+82 \mathrm{~km} \mathrm{~s}^{-1}$. Nonetheless, an absorption feature at $-58 \mathrm{~km} \mathrm{~s}^{-1}$ is possibly detectable and somewhat resolved on the low-velocity edge of the $+64 \mathrm{~km} \mathrm{~s}^{-1}$ component of cis- $\mathrm{CH}_{2} \mathrm{OHCHO}$. This profile is also not inconsistent with there being line absorption by $l-\mathrm{C}_{3} \mathrm{H}$ at $-47 \mathrm{~km} \mathrm{~s}^{-1}$, although it is not possible to claim this feature is detected. However, if absorption by the $(F=2-1 l=e)$ transition is present at -58 and $-47 \mathrm{~km} \mathrm{~s}^{-1}$, the $(F=1-0)$ hyperfine components would occur at -80 and $-73 \mathrm{~km} \mathrm{~s}^{-1}$, and would account for all absorption at these velocities. Because the -73 and $-80 \mathrm{~km} \mathrm{~s}^{-1}$ components were detected in the $(l=f)$ component, we are inclined to believe that the absorption at -80 and $-73 \mathrm{~km} \mathrm{~s}^{-1}$ is from the -80 and $-73 \mathrm{~km} \mathrm{~s}^{-1}$ clouds, and are not hyperfine features from the -58 and $-47 \mathrm{~km} \mathrm{~s}^{-1}$ clouds. Therefore, we treat the features at -48 and $-57 \mathrm{~km} \mathrm{~s}^{-1}$ in the $(l=f)$ profile at $32627 \mathrm{MHz}$ as unidentified lines but warn of the possibility that the absorption is from the line-of-sight clouds.

In the line profile of $l-\mathrm{C}_{3} \mathrm{H}^{+}(2-1)$, line absorption is clearly detected within the $0 \mathrm{~km} \mathrm{~s}^{-1}$ cloud, and absorption is also present in the $-106,-73$, and $-47 \mathrm{~km} \mathrm{~s}^{-1}$ clouds. An unidentified emission line at $44994 \mathrm{MHz}$ in the rest frame of Sgr B2 prevents characterization of the $-40 \mathrm{~km} \mathrm{~s}^{-1}$ cloud. In the (1$0)$ transition of $l-\mathrm{C}_{3} \mathrm{H}^{+}$(Appendix A), absorption is also clearly detected at $\sim 0 \mathrm{~km} \mathrm{~s}^{-1}$, and the profile provides evidence supporting the detection of $l-\mathrm{C}_{3} \mathrm{H}^{+}$in the $-73 \mathrm{~km} \mathrm{~s}^{-1}$ cloud. The detection of $l-\mathrm{C}_{3} \mathrm{H}^{+}$in the $-106 \mathrm{~km} \mathrm{~s}^{-1}$ cloud remains somewhat suspect, as it either contains substantial absorption from -120 to $-110 \mathrm{~km} \mathrm{~s}^{-1}$ or is confused with a different line or with baseline effects. We note that the baseline is stable on either side of the velocity range of interest, so do not favor the latter explanation. However, as the $-106 \mathrm{~km} \mathrm{~s}^{-1}$ component is too weak to be detected in the (1-0) transition, we treat $l-\mathrm{C}_{3} \mathrm{H}^{+}$in the $-106 \mathrm{~km} \mathrm{~s}^{-1}$ cloud tentatively, and recommend that it should be confirmed with an additional transition.

\subsection{Cloud kinematics}

Figure 6 shows the Gaussian fit parameters of center velocity and line width for all components fit to selected line profiles. In this figure, it is apparent that the $-106 \mathrm{~km} \mathrm{~s}^{-1}$ component has particularly consistent and well defined parameters of line center and width, at $v \approx-105.7 \mathrm{~km} \mathrm{~s}^{-1}$ and $\Delta v \approx 3.1 \mathrm{~km} \mathrm{~s}^{-1}$. The standard deviations on these parameters, of 0.2 and 0.3 , respectively, are consistent with the errors to individual measurements. Although the profile of $\mathrm{H}_{2} \mathrm{CO}\left(1_{10}-1_{11}\right)$ includes a weak broad component at or near $-106 \mathrm{~km} \mathrm{~s}^{-1}$, this line and all other transitions shown in Fig. 6 have absorption at $(v, \Delta v) \approx\left(-105.7 \mathrm{~km} \mathrm{~s}^{-1}, 3 \mathrm{~km} \mathrm{~s}^{-1}\right)$. For some high $\mathrm{S} / \mathrm{N}$ transitions, a narrow wing is present to the positive velocity side of the $-106 \mathrm{~km} \mathrm{~s}^{-1}$ cloud, at approximately $-102 \mathrm{~km} \mathrm{~s}^{-1}$, and a moderately broad wing is present toward the negative velocity side, near $-115 \mathrm{~km} \mathrm{~s}^{-1}$.
The $-92 \mathrm{~km} \mathrm{~s}^{-1}$ cloud is centered at $-92.6 \mathrm{~km} \mathrm{~s}^{-1}$ and has mean width of $\Delta v=2.7 \mathrm{~km} \mathrm{~s}^{-1}$. The line widths vary significantly more for the $-92 \mathrm{~km} \mathrm{~s}^{-1}$ cloud than for the $-106 \mathrm{~km} \mathrm{~s}^{-1}$ cloud, with measured values ranging from $\Delta v=1$ to $5 \mathrm{~km} \mathrm{~s}^{-1}$.

In the $-80 \mathrm{~km} \mathrm{~s}^{-1}$ cloud, most line fits are tightly clustered around $v \approx 80.4 \mathrm{~km} \mathrm{~s}^{-1}$ and $\Delta v \approx 3.5 \mathrm{~km} \mathrm{~s}^{-1}$. Absorption by the $-73 \mathrm{~km} \mathrm{~s}^{-1}$ cloud typically has a larger line width, with a median value of $4.9 \mathrm{~km} \mathrm{~s}^{-1}$. Whereas lines of $\mathrm{SiO}$, and $\mathrm{H}_{2} \mathrm{CO}$ have higher line widths, of 9-12 $\mathrm{km} \mathrm{s}^{-1}$, most other species have widths of $\sim 5 \mathrm{~km} \mathrm{~s}^{-1}$, although significant scatter exists.

The $-58 \mathrm{~km} \mathrm{~s}^{-1}$ cloud is centered at $-57.6 \mathrm{~km} \mathrm{~s}^{-1}$, and has a line width of $\sim 2.1 \mathrm{~km} \mathrm{~s}^{-1}$ with statistically significant scatter.

The $-47 \mathrm{~km} \mathrm{~s}^{-1}$ cloud consists of two narrow velocity components, at -46.6 and $-44 \mathrm{~km} \mathrm{~s}^{-1}$, in addition to a broad component that is prominent in transitions of $c-\mathrm{C}_{3} \mathrm{H}_{2}$ and $\mathrm{H}_{2} \mathrm{CO}$. The narrow components have very consistent line center velocities, and typical widths of 0.7 and $1.0 \mathrm{~km} \mathrm{~s}^{-1}$ at -46.6 and $-44 \mathrm{~km} \mathrm{~s}^{-1}$, respectively. Similarly, for small species with high $\mathrm{S} / \mathrm{N}$, the $-40 \mathrm{~km} \mathrm{~s}^{-1}$ component consists of two narrow lines, at -40.8 and $-38.7 \mathrm{~km} \mathrm{~s}^{-1}$, with widths of 1.9 and $1.5 \mathrm{~km} \mathrm{~s}^{-1}$, respectively. For lower $\mathrm{S} / \mathrm{N}$ transitions, the two components are fit with a single Gaussian with a higher line width.

As observed in the highest $\mathrm{S} / \mathrm{N}$ transitions, the $-23 \mathrm{~km} \mathrm{~s}^{-1}$ cloud consists of many narrow features superimposed on two moderately broad features which dominate the integrated flux. These two moderately broad features are at -27.1 and $-20.1 \mathrm{~km} \mathrm{~s}^{-1}$, with widths of 4.8 and $4.6 \mathrm{~km} \mathrm{~s}^{-1}$, respectively, although significant scatter exists.

Finally, the $\sim 0$ and $+20 \mathrm{~km} \mathrm{~s}^{-1}$ clouds contain multiple superimposed components, including broad profiles $\left(\Delta v>10 \mathrm{~km} \mathrm{~s}^{-1}\right)$ with moderately broad $\left(3<\Delta v<10 \mathrm{~km} \mathrm{~s}^{-1}\right)$ and narrow $\left(\Delta v<3 \mathrm{~km} \mathrm{~s}^{-1}\right)$ features superimposed. Fits to this material typically include a feature at $-1.8 \mathrm{~km} \mathrm{~s}^{-1}$, a strong component at $+3.5 \mathrm{~km} \mathrm{~s}^{-1}$, a component at $+9 \mathrm{~km} \mathrm{~s}^{-1}$, a broad component centered between $10<v<15 \mathrm{~km} \mathrm{~s}^{-1}$, and a broad feature at $+30 \mathrm{~km} \mathrm{~s}^{-1}$. The exact profiles vary significantly in different lines however, producing substantial scatter in Fig. 6.

\subsection{Molecular column density measurements}

We determined column densities of every molecule in each distiguishable cloud component. To do so, we obtained the integrated line optical depths by

$$
\begin{array}{r}
\int \tau_{l} \mathrm{~d} v=\int_{v_{0}}^{v_{1}}-\ln \left(1+\frac{T_{\mathrm{L}}}{T_{\mathrm{C}}-\left[f\left(T_{\mathrm{Ex}}\right)-f\left(T_{\mathrm{CMB}}\right)\right]}\right) \mathrm{d} v ; \\
f(T)=\frac{h v / k}{\mathrm{e}^{h v / k T}-1} \leq T,
\end{array}
$$

(Nyman 1984; Greaves \& Nyman 1996) with $T_{\mathrm{CMB}}=2.73 \mathrm{~K}$ over the bounding velocities $v_{0}$ and $v_{1}$ provided in Table 2 for clouds at $-120,-106,-92,-80,-73,-58,-46,-40,-23, \sim 0$, and $+20 \mathrm{~km} \mathrm{~s}^{-1}$. We convert from integrated line optical depths to column densities by:

$$
N=8.0 \times 10^{12} \frac{Q}{S_{i j} \mu^{2}} \frac{\int \tau \mathrm{d} v}{\mathrm{e}^{-E_{\mathrm{L}} / k T_{\mathrm{Ex}}}-\mathrm{e}^{-E_{\mathrm{U}} / k T_{\mathrm{Ex}}}} \mathrm{cm}^{-2},
$$

by the conventions used in Lucas \& Liszt (1993) and Greaves \& Nyman (1996), where $Q$ is the partition function, $E_{\mathrm{L}}$ and $E_{\mathrm{U}}$ are the upper and lower state energies, $S_{i j}$ is the intrinsic line strength, and $\mu$ is the transition dipole moment in Debye. 


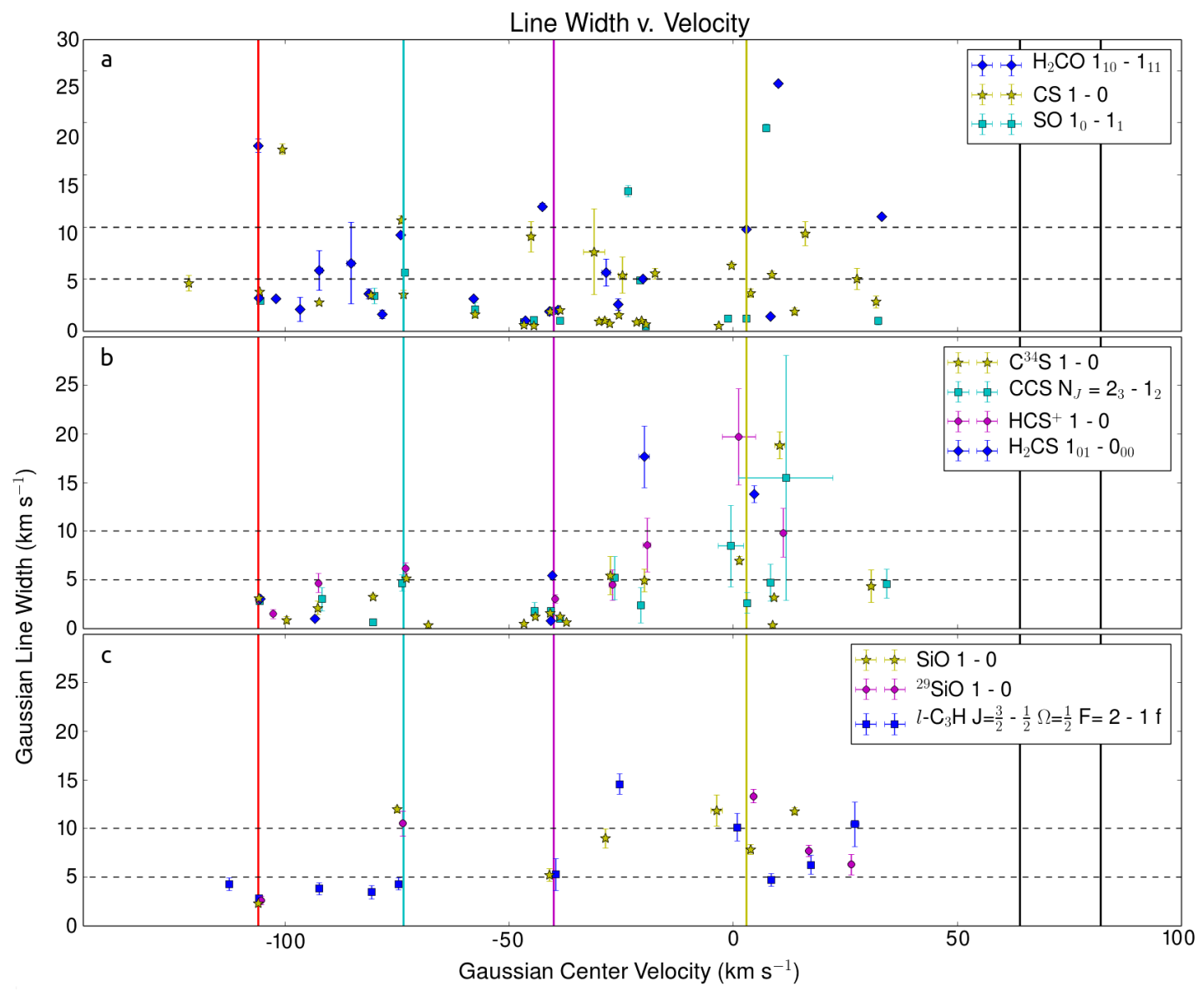

Fig. 6. Gaussian fit parameters of center velocity and line width for individual Gaussian components fit to selected transitions of $a$ ) $\mathrm{H}_{2} \mathrm{CO}, \mathrm{CS}$, and $\mathrm{SO}, b)$ CS-bearing molecules, and $c$ ) isotopologues of $\mathrm{SiO}$ and $l-\mathrm{C}_{3} \mathrm{H}$. In all panels, black vertical lines indicate the velocities of line absorption by Sgr B2 at +64 and $+82 \mathrm{~km} \mathrm{~s}^{-1}$, and colored lines are located at $-106,-73.5,-40$, and $+3 \mathrm{~km} \mathrm{~s}^{-1}$. Horizontal dashed lines indicate line widths of $10 \mathrm{~km} \mathrm{~s}^{-1}$ and $5 \mathrm{~km} \mathrm{~s}^{-1}$.

The calculation assumed that the absorbing gas extends homogenously over the continuum. This assumption is standard in studies of diffuse and translucent gas in line-of-sight absorption (e.g., Greaves \& Nyman 1996; Lucas \& Liszt 2002; Qin et al. 2010).

While the value of the partition function is typically estimated from equations like those provided in McDowell (1988, 1990), these equations diverge from the true values at low excitation temperatures of $T_{\mathrm{ex}}<10 \mathrm{~K}$. Due to the extremely low excitation temperatures in the line-of-sight clouds, we determined the partition functions directly by counting states with $Q=\Sigma g \mathrm{e}^{-E / k T_{\text {ex }}}$. For this computation and for input line parameters required in Eq. (2), line data from the Cologne Database of Molecular Spectroscopy (CDMS; Müller et al. 2005) and the NASA Jet Propulsion Laboratory catalog (JPL; Pickett et al. 1998) were accessed through the ALMA Spectral Line Catalog Splatalogue ${ }^{2}$. In the data output from the CDMS and JPL catalogs, the value $S_{i j} \mu^{2}$ incorporates the degeneracy of the upper state $g_{\mathrm{U}}$. The value of the degeneracy varies depending on how states are defined, in particular whether hyperfine states and torsionally excited states are treated individually or collapsed. As a result, care must be taken to ensure consistency between the adopted or calculated value of the partition function and the line parameters used for the column density calculation. In our work, the partition functions were computed directly from the line data at $T=3 \mathrm{~K}$ using all states with energy $E<100 \mathrm{~K}$ (and up to

\footnotetext{
2 The ALMA Spectral Line Catalog is available at www.splatalogue.net; (Remijan et al. 2007)
}

$300 \mathrm{~K}$ for molecules with fewer transitions). We further verified that our calculated values of the partition function are in agreement with the values published by the catalogs at temperatures of $9.375,18.75$, and $37.5 \mathrm{~K}$.

We determined errors to the integrated line optical depth measurements by including error contributions from three sources added in quadrature. These included (1) error to the integrated line optical depth estimated by the root mean squared residual between the line profile fit and the data times the width of the velocity range considered; (2) a $2.5 \mathrm{mK}$ baseline uncertainty, which is a typical level of variation in the baseline offset within a single line profile; and (3) an estimated 5 percent uncertainty in the denominator of Eq. (1).

We determined that a model with $T_{\mathrm{ex}}=3 \mathrm{~K}$ does a good job of accounting for the line absorption for nearly all molecules in nearly all components. Initially, for molecules with multiple transitions in our data set, we allowed both $T_{\text {ex }}$ and $N_{\text {molec }}$ to vary and determined that values of $2.5-3.7 \mathrm{~K}$ produced the best fits. After determining that $3 \mathrm{~K}$ is appropriate, we set $T_{\mathrm{ex}}=3 \mathrm{~K}$ before obtaining the best-fit values for the molecular column density. For illustration, Fig. 7 shows the modeled and observed values of the integrated line optical depth obtained with $T_{\mathrm{ex}}=3 \mathrm{~K}$ for observed transitions of CCS, $c-\mathrm{C}_{3} \mathrm{H}_{2}$, and $\mathrm{H}_{2} \mathrm{CO}$. For nearly all transitions, the best fit was within $2 \epsilon$ of the observed value. Further, for most molecules with multiple transitions (namely $\mathrm{OH}, \mathrm{CCS}, l-\mathrm{C}_{3} \mathrm{H}, l-\mathrm{C}_{3} \mathrm{H}^{+}, c-\mathrm{C}_{3} \mathrm{H}_{2}$, and $\mathrm{H}_{2} \mathrm{CO}$ ), the values of $N_{\text {molec }}$ estimated from different transitions vary by $\lesssim 10$ percent, 


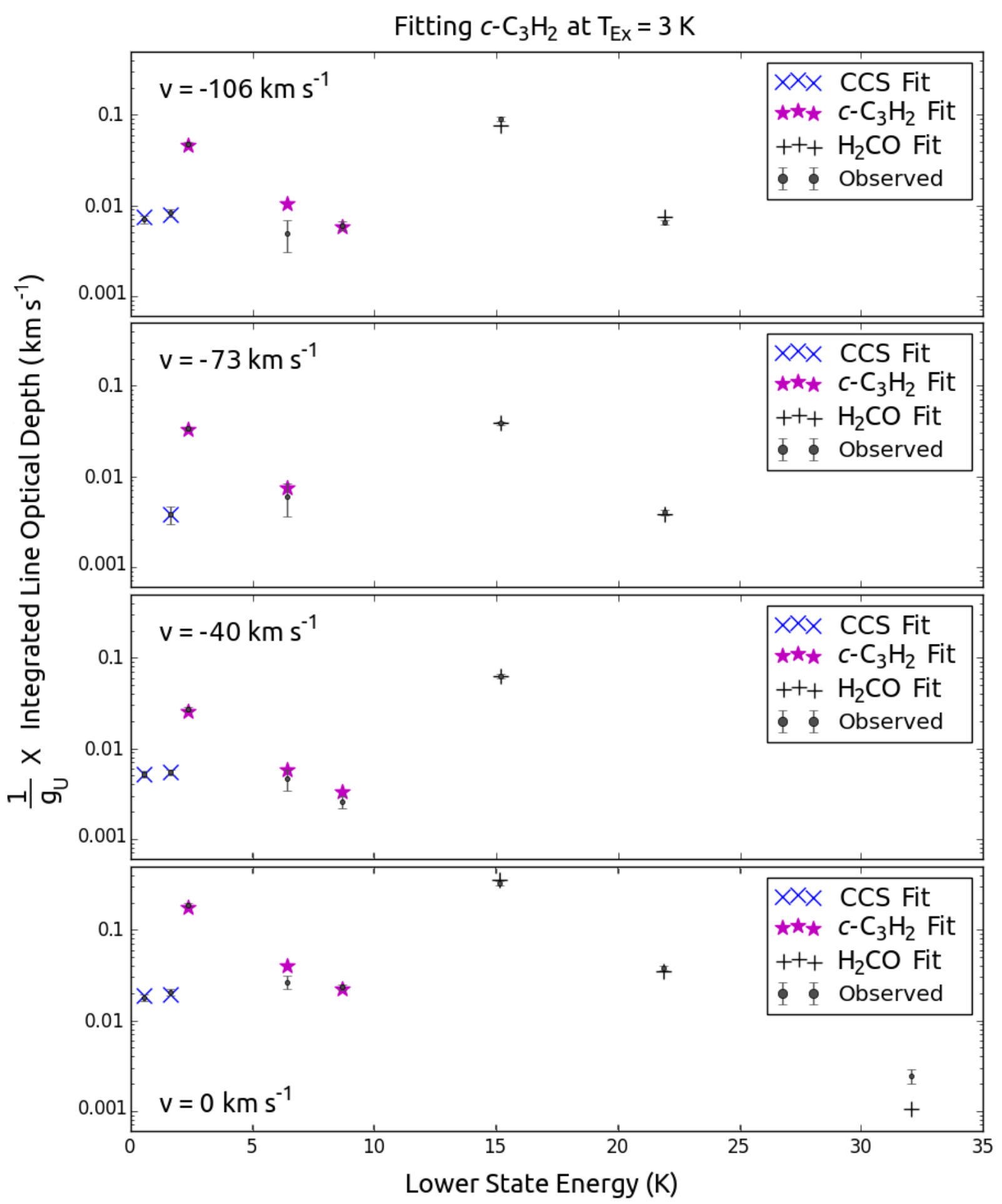

Fig. 7. Observed integrated line optical depths and best fit models, computed at $T_{\mathrm{ex}}=3 \mathrm{~K}$, of CCS, $c$ - $\mathrm{C}_{3} \mathrm{H}_{2}$, and $\mathrm{H}_{2} \mathrm{CO}$. Error bars indicate $1 \sigma$ errors.

and somewhat larger variation is observed for a few velocity components of $c-\mathrm{C}_{3} \mathrm{H}_{2}$ and $\mathrm{H}_{2} \mathrm{CO}$.

For molecules with only a single transition observed in lineof-sight absorption (i.e., SiO, SO, and CS-bearing species), it was then straightforward to determine the molecular column density by Eq. (2) with the assumption of $T_{\mathrm{ex}}=3 \mathrm{~K}$. The column density errors reported include the error from the integrated optical depth measurement as described above, and do not include uncertainty in the excitation temperature.
For molecules with multiple transitions, we determined the reported column density as the best fit to all measured transitions as determined by an error-weighted least-squares method. The errors for individual transitions input to the column density fitting procedure include the three contributions specified above. For column density estimates determined with multiple transitions, it was possible to estimate the total error by two methods. The first method included errors to the individual fits and estimated that the column density fit error will decreases with a 
Table 3. Molecular and neutral hydrogen column density estimates in units of $10^{21} \mathrm{~cm}^{-2}$.

\begin{tabular}{|c|c|c|c|c|c|c|c|c|c|c|c|c|}
\hline \multirow[t]{2}{*}{ Parameter } & \multirow[t]{2}{*}{ Position } & \multicolumn{11}{|c|}{ Absorption cloud velocity $\left(\mathrm{km} \mathrm{s}^{-1}\right)$} \\
\hline & & -120 & -106 & -92 & -80 & -73 & -58 & -47 & -40 & -23 & 0 & +20 \\
\hline $\mathrm{N}\left(\mathrm{H}_{2}\right)$ & $(\mathrm{N})$ & $0.17(2)$ & $3.2(2)$ & $1.72(8)$ & $1.12(6)$ & $2.3(1)$ & $0.52(3)$ & $1.4(1)$ & $1.8(2)$ & $3.5(4)$ & 12(1) & $3.2(5)$ \\
\hline $\mathrm{N}\left(\mathrm{H}_{2}\right)^{[1]}$ & $(\mathrm{N})$ & & $4.8(5)$ & & \multicolumn{2}{|c|}{$2.9(1)$} & $0.48(3)$ & \multicolumn{2}{|c|}{$3.0(1)$} & $3.1(4)$ & \multicolumn{2}{|c|}{$14.6(16)$} \\
\hline $\mathrm{N}\left(\mathrm{H}_{2}\right)^{[2]}$ & (M) & & 6 & & \multicolumn{2}{|c|}{1.2} & 0.9 & \multicolumn{2}{|c|}{9} & 3 & \multicolumn{2}{|c|}{16} \\
\hline $\mathrm{N}\left(\mathrm{H}_{2}\right)^{[3]}$ & (M) & & 5 & & & & & \multicolumn{2}{|c|}{9} & 4 & \multicolumn{2}{|c|}{14} \\
\hline $\mathrm{N}(\mathrm{HI})^{[4]}$ & $(\mathrm{N})$ & \multicolumn{5}{|c|}{$1.0(3)$} & \multicolumn{3}{|c|}{$1.5(6)$} & $4.0(6)$ & & \\
\hline$f\left(\mathrm{H}_{2}\right)$ & $(\mathrm{N})$ & \multicolumn{5}{|c|}{$0.94(2)$} & \multicolumn{3}{|c|}{$0.84(5)$} & $0.65(3)$ & & \\
\hline$A_{\mathrm{V} 0}{ }^{[5]} \approx$ & $(\mathrm{N})$ & 0.08 & 1.5 & 0.8 & 0.5 & 1.1 & 0.28 & 0.76 & 0.96 & 2.5 & $>4.2$ & $>2.4$ \\
\hline
\end{tabular}

Notes. [1] Measurements are computed from PRIMOS observations in the velocity ranges listed in Greaves \& Nyman (1996). [2-3] Values toward Sgr B2(M) from [2] Greaves \& Nyman (1996) by conversion from $\mathrm{H}^{13} \mathrm{CO}^{+}$and [3] Irvine et al. (1987) by conversion from ${ }^{13} \mathrm{CO}$. [4] Values toward Sgr B2(N) from Indriolo et al. (2015) from $21 \mathrm{~cm} \mathrm{HI}$ absorption. [5] Central extinction of the cloud, estimated by $2 A_{\mathrm{V} 0}=A_{\mathrm{V}}=$ $\left(N_{\mathrm{HI}}+2 N_{\mathrm{H}_{2}}\right) /\left(2.2 \times 10^{21} \mathrm{~cm}^{-2}\right)$, from (Güver \& Özel 2009).

greater number of constraints, by

$\epsilon_{\mathrm{N}}^{2}=\left(\sum \frac{1}{\epsilon_{i}^{2}}\right)^{-1}$,

for the error to the column density $\epsilon_{\mathrm{N}}$ and errors to individual fits $\epsilon_{i}$. In the second method, the error was estimated by measuring the agreement between the predicted and observed integrated optical depths, weighted by the errors on individual transitions, by

$\epsilon_{\mathrm{N}}^{2}=\sum\left(\frac{1}{\epsilon_{i}^{2}} \times\left(\frac{\mathrm{O}_{i}-\mathrm{P}_{i}}{\mathrm{P}_{i}}\right)^{2}\right)$,

for the error to the column density $\left(\epsilon_{\mathrm{N}}\right)$, errors to individual fits $\left(\epsilon_{i}\right)$, observed integrated line optical depths $\left(\mathrm{O}_{i}\right)$, and predicted integrated line optical depths from the best fit model $\left(\mathrm{P}_{i}\right)$. If the errors estimated for the individual transitions are appropriate, then the two methods should return similar values. We generally observe good agreement between estimates made by these two methods, and we report the larger value estimated by the two methods. The best fit molecular column densities and error estimates are provided in Appendix A, and most column density errors are of order 10 percent.

\subsection{Hydrogen column density measurements}

In order to determine abundances relative to $\mathrm{H}_{2}$, we estimated the hydrogen column in each component assuming that the abundance of $c-\mathrm{C}_{3} \mathrm{H}_{2}$ is constant and equal to $X=\frac{N_{\mathrm{c}^{-} \mathrm{H}_{2}}}{\mathrm{~N}_{\mathrm{H}_{2}}}=$ $2.5 \times 10^{-9}$. This assumption is based on the work of Liszt et al. (2012), which found that $X_{c-\mathrm{C}_{3} \mathrm{H}_{2}}=(2-3) \times 10^{-9}$ with little variation in diffuse clouds in the Galactic disk, and in additional studies of hydrocarbon ratios in diffuse and translucent clouds throughout the Galaxy (Lucas \& Liszt 2000; Sheffer et al. 2008; Gerin et al. 2010b, 2011). In Sect. 4.1 we argue that this is the best method for estimating the molecular hydrogen column in the PRIMOS data. The resulting hydrogen columns are provided in Table 3. The hydrogen columns estimated by conversion from $c-\mathrm{C}_{3} \mathrm{H}_{2}$ column densities are, for most clouds, similar to previous estimates made in the line-of-sight toward Sgr B2(M) located $\sim 45$ arcsec south. Errors to the molecular hydrogen column come from the estimated errors to the column densities of
$c-\mathrm{C}_{3} \mathrm{H}_{2}$, and do not include possible variation in the abundance of $c-\mathrm{C}_{3} \mathrm{H}_{2}$.

For the sake of comparison, we also include the molecular hydrogen columns measured by previous authors toward Sgr B2(M), located 45 arcsec south of (N), by conversion from $\mathrm{H}^{13} \mathrm{CO}^{+}$and ${ }^{13} \mathrm{CO}$ (Greaves \& Nyman 1996; Irvine et al. 1987). Although clouds are present at approximately the same velocities in the two sightlines, the relative absorption strengths in the different kinematic components vary. As such, our estimates of the molecular hydrogen column density are not expected to match perfectly. However, we do see approximate agreement for most of the clouds, providing one indication that our conversion from $N_{c-\mathrm{C}_{3} \mathrm{H}_{2}}$ to $N_{\mathrm{H}_{2}}$ is appropriate.

Additionally, Table 3 includes the neutral hydrogen column densities reported by Indriolo et al. (2015) in the sightline to Sgr B2(N). These were measured by HI absorption in the $21-\mathrm{cm}$ line observed with the Effelsberg 100-m telescope (Winkel et al. 2016). While Indriolo et al. (2015) reported column densities over velocity ranges that include multiple distinct clouds as observed by $c-\mathrm{C}_{3} \mathrm{H}_{2}$, dramatic trends are present in the molecular fractions measured. The molecular fraction in the $-23 \mathrm{~km} \mathrm{~s}^{-1}$ cloud, located in the disk, is 0.65 , somewhat larger than typical values of 0.4 measured in other diffuse clouds that contain heavy molecules (Liszt et al. 2012). In the velocity range dominated by gas in the $3 \mathrm{kpc}$ arm, $\sim 85$ percent of the hydrogen is in molecular form, and an even higher molecular fraction is measured in the velocity range dominated by Galactic center gas, with $>90$ percent of the hydrogen in molecular form. In the final row of Table 3, we estimate the central extinctions in individual clouds by combining the $\mathrm{H}_{2}$ column density measurements reported in the first row with HI column densities estimated using the approximate molecular fractions. The total extinction in the cloud is estimated with $A_{\mathrm{V}}=1$ corresponding to

$N_{\mathrm{HI}}+2 N_{\mathrm{H} 2}=2.2 \times 10^{21} \mathrm{~cm}^{-2}$,

from (Güver \& Özel 2009), and the central extinction $\left(A_{\mathrm{V}_{0}}\right)$ is half the total extinction. The extinction estimates should be adopted as approximate values, as the true extinction within the clouds depends on multiple factors as discussed in Sect. 4.2.

\subsection{Abundance patterns}

Using the molecular hydrogen columns in the first row of Table 3, we convert molecular column densities to abundances 


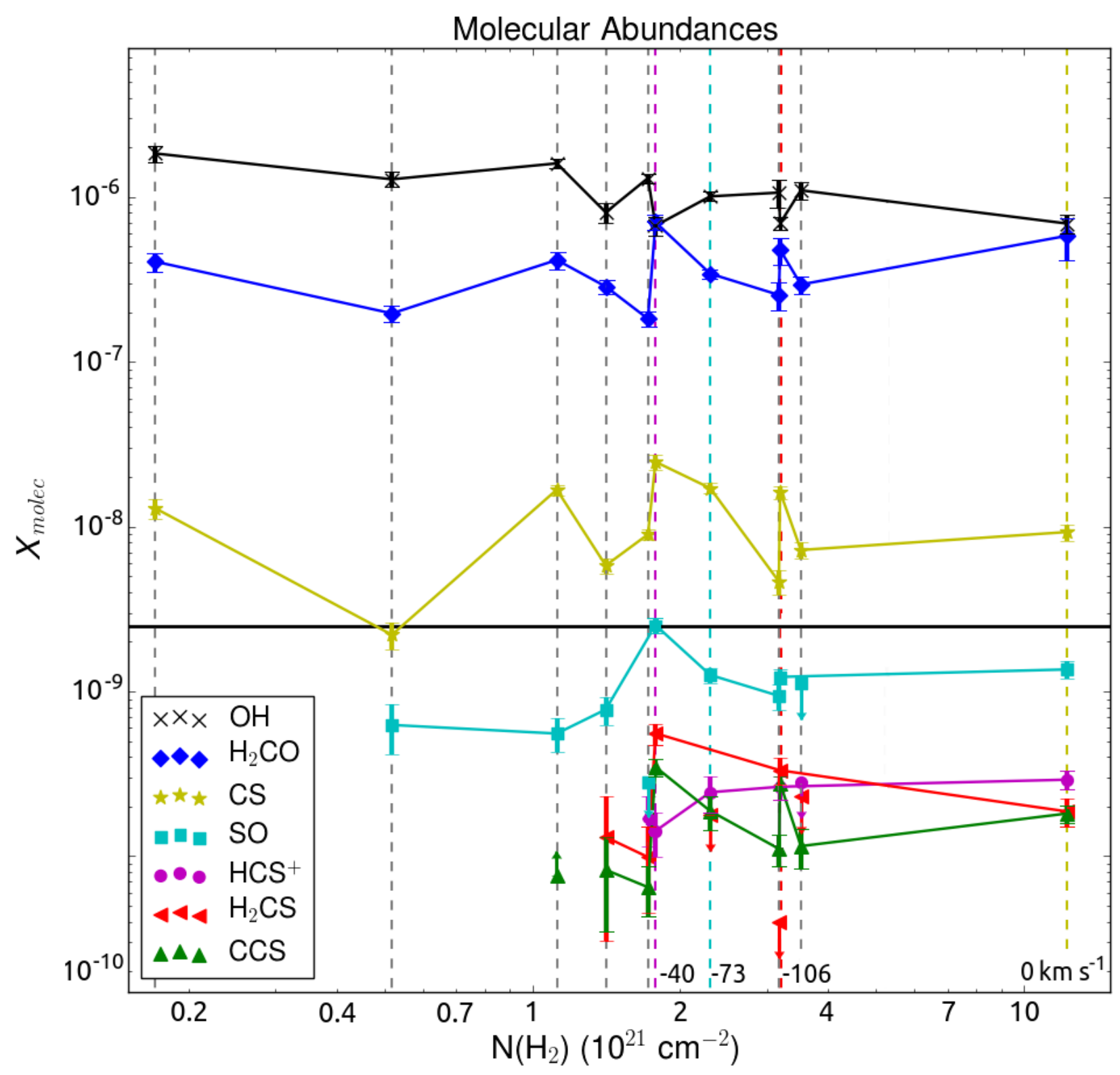

Fig. 8. Molecular abundances of $\mathrm{OH}, \mathrm{H}_{2} \mathrm{CO}, \mathrm{SO}$, and CS-bearing species. The black solid line represents the assumed abundance of $c-\mathrm{C}_{3} \mathrm{H}_{2}$ used for the abundance determination. In order from lowest to highest hydrogen column, the $-120,-58,-80,-47,-92,-40,-73,+20,-106,-23$, and $\sim 0 \mathrm{~km} \mathrm{~s}^{-1}$ components are marked with vertical dashed lines. Colored vertical lines, as labeled, mark the $-106,-73,-40$, and $0 \mathrm{~km} \mathrm{~s}^{-1}$ clouds.

with respect to $\mathrm{H}_{2}$. The resulting abundances are provided in Table 4 and plotted against $N_{\mathrm{H}_{2}}$ in Figs. 8 and 9. Abundance errors are estimated as the fractional error on the measured molecular column added in quadrature with the fractional error on the molecular hydrogen column for each velocity component, resulting in typical errors of 10 to 20 percent. The abundance results are summarized for each molecule below.

\subsubsection{OH}

The $\left(J=\frac{3}{2} \Omega=\frac{3}{2} F=1^{+}-1^{-}\right)$and $\left(J=\frac{3}{2} \Omega=\frac{3}{2} F=2^{+}-2^{-}\right)$ transitions of $\mathrm{OH}$ at 1665 and $1667 \mathrm{MHz}$ have extremely similar line profiles and very consistent values of $N_{\text {molec }}$ are derived using the two lines, with measurements nearly always within 8 percent of one another. In Table A.1, we report values obtained from a best fit to these two lines and obtain values of order
$10^{-6}$. The $\left(J=\frac{3}{2} \Omega=\frac{3}{2} F=1^{+}-2^{-}\right)$line profile at $1612 \mathrm{MHz}$ appears somewhat different however. Column density measurements made using the $1612 \mathrm{MHz}$ transition are slightly higher, by a factor of 1.5 to 3 , than the values reported in Table A.1 for the clouds at $-120,-58,-46,-40,-23,0$, and $+25 \mathrm{~km} \mathrm{~s}^{-1}$ clouds. The column density estimate is slightly lower, by a factor of 3 , in the $-92 \mathrm{~km} \mathrm{~s}^{-1}$ cloud, and an order of magnitude lower than the reported values at -80 and $-73 \mathrm{~km} \mathrm{~s}^{-1}$. OH does not appear to occupy the same volume of gas as $c-\mathrm{C}_{3} \mathrm{H}_{2}$, as evidenced by the differences in the line profiles, so it is not appropriate to interpret the abundances reported in Table 4 as true abundances. However, it is noteable that while statistically significant scatter exists, the values of $N_{\mathrm{OH}} / N_{\mathrm{H}_{2}}$ typically decrease with $N_{\mathrm{H}_{2}}$, as is apparent from the negative slope for $\mathrm{OH}$ in Fig. 8. We include the values as they likely have physical significance as discussed in Sect. 5.3.1. 


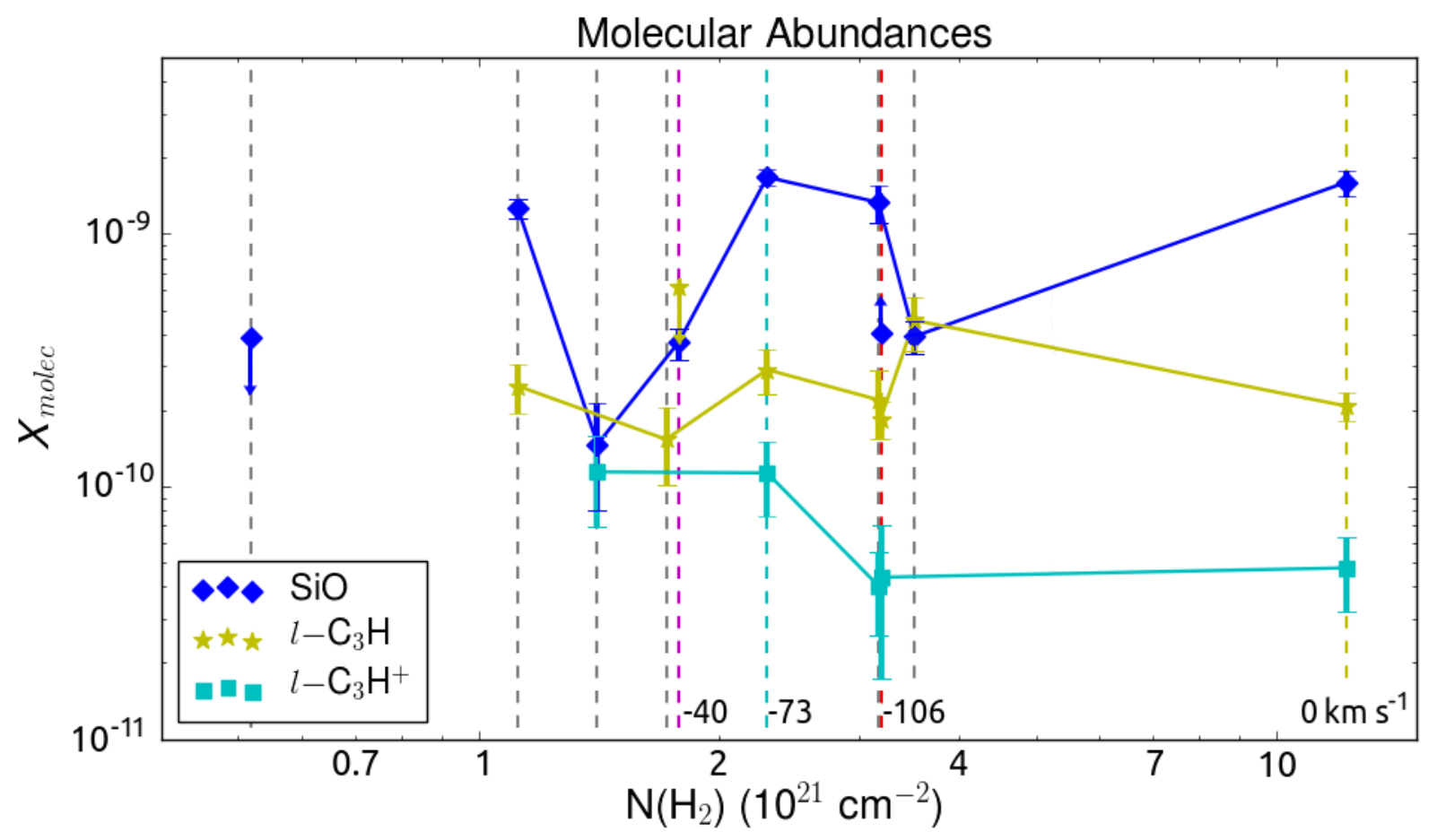

Fig. 9. Molecular abundances of $\mathrm{SiO}, l-\mathrm{C}_{3} \mathrm{H}$, and $l-\mathrm{C}_{3} \mathrm{H}^{+}$. In order from lowest to highest hydrogen column, the $-58,-80,-47,-92,-40,-73$, $+20,-106,-23$, and $\sim 0 \mathrm{~km} \mathrm{~s}^{-1}$ components are marked with vertical dashed lines. Colored vertical lines, as labeled, mark the $-106,-73,-40$, and $0 \mathrm{~km} \mathrm{~s}^{-1}$ clouds.

Table 4. Derived molecular abundances relative to $\mathrm{H}_{2}$.

\begin{tabular}{|c|c|c|c|c|c|c|c|c|c|c|c|c|}
\hline \multirow[t]{2}{*}{ Molecule } & \multicolumn{11}{|c|}{ Absorption cloud velocity $\left(\mathrm{km} \mathrm{s}^{-1}\right)$} & \multirow[t]{2}{*}{ Units } \\
\hline & -120 & -106 & -92 & -80 & -73 & -58 & -47 & -40 & -23 & 0 & +20 & \\
\hline $\mathrm{OH}$ & $1.8(2)$ & $0.69(6)$ & $1.30(8)$ & $1.6(1)$ & $1.01(7)$ & $1.3(1)$ & $0.8(1)$ & $0.67(8)$ & $1.1(1)$ & $0.69(9)$ & $1.1(2)$ & $10^{-6}$ \\
\hline$c-\mathrm{C}_{3} \mathrm{H}_{2}{ }^{[1]}$ & 2.5 & 2.5 & 2.5 & 2.5 & 2.5 & 2.5 & 2.5 & 2.5 & 2.5 & 2.5 & 2.5 & $10^{-9}$ \\
\hline$c-\mathrm{H}^{13} \mathrm{CCCH}$ & & $1.0(2)$ & $1.0(3)$ & & $0.6(4)$ & & & $<0.3$ & $0.2(1)$ & $0.9(2)$ & $1.0(2)$ & $10^{-10}$ \\
\hline $\mathrm{H}_{2} \mathrm{CO}$ & $4.1(5)$ & $4.8(9)$ & $1.8(2)$ & $4.1(5)$ & $3.4(2)$ & $2.0(2)$ & $2.9(3)$ & $7.0(7)$ & $2.9(4)$ & $5.8(16)$ & $2.5(5)$ & $10^{-7}$ \\
\hline $\mathrm{H}_{2}^{13} \mathrm{CO}$ & & $8.4(9)$ & & $4.5(9)$ & $3.4(13)$ & & & $10(4)$ & $6.2(11)$ & $10(1)$ & & $10^{-9}$ \\
\hline SO & & 1.2(1) & $<0.3$ & $0.6(1)$ & $1.3(1)$ & $0.6(2)$ & $0.8(2)$ & $2.5(3)$ & $1.1(3)$ & $1.4(2)$ & $0.9(2)$ & $10^{-9}$ \\
\hline $\mathrm{CS}$ & $1.3(2)$ & $1.6(1)^{[2]}$ & $0.90(6)$ & $1.7(1)$ & $1.7(1)$ & $0.22(4)$ & $0.58(6)$ & $2.5(3)^{[2]}$ & $0.72(8)$ & $0.9(1)^{[2]}$ & $0.46(8)$ & $10^{-8}$ \\
\hline $\mathrm{C}^{34} \mathrm{~S}$ & & $1.9(2)$ & $0.3(1)$ & $2.0(2)$ & $2.6(2)$ & $<1.2$ & $0.6(2)$ & $3.6(4)$ & $0.7(1)$ & $1.7(2)$ & $0.8(1)$ & $10^{-9}$ \\
\hline CCS & & $2.8(3)$ & $0.6(2)$ & $\gtrsim 0.8$ & $1.9(4)$ & & $\gtrsim 0.8$ & $3.5(4)$ & $1.2(3)$ & $1.8(2)$ & $1.1(2)$ & $10^{-10}$ \\
\hline $\mathrm{HCS}^{+}$ & & $2.6(4)$ & $1.7(6)$ & & $2.4(6)$ & & & $1.4(4)$ & $\lesssim 2.8$ & $2.9(4)$ & & $10^{-10}$ \\
\hline $\mathrm{H}_{2} \mathrm{CS}$ & & $3.3(6)$ & $1.0(5)$ & & $\leq 1.4$ & & 1.3(1) & $5.6(8)$ & $\lesssim 2.3$ & $1.9(4)$ & $\lesssim 0.3$ & $10^{-10}$ \\
\hline $\mathrm{SiO}$ & & $>0.4$ & & $1.3(1)^{[3]}$ & $1.7(1)$ & $<0.4$ & $0.15(7)^{[3]}$ & $0.37(5)$ & $0.39(6)$ & $1.6(2)$ & $1.3(2)$ & $10^{-9}$ \\
\hline${ }^{29} \mathrm{SiO}$ & & $3.6(12)$ & & $4(3)^{[3]}$ & $9(2)$ & & & & & $12(2)$ & $11(2)$ & $10^{-11}$ \\
\hline${ }^{30} \mathrm{SiO}$ & & $2.2(9)$ & & & & & & & & & & $10^{-11}$ \\
\hline$l-\mathrm{C}_{3} \mathrm{H}$ & & $1.9(3)$ & $1.5(5)$ & $2.5(5)$ & $2.9(6)$ & & & $\lesssim 6.1$ & $4.6(11)$ & $2.1(3)$ & $2.2(6)$ & $10^{-10}$ \\
\hline$l-\mathrm{C}_{3} \mathrm{H}^{+}$ & & $4.4(3)$ & & & $11(4)$ & & $11(4)$ & & & $4.8(2)$ & $4.0(1)$ & $10^{-11}$ \\
\hline
\end{tabular}

Notes. [1] The abundance of $c-\mathrm{C}_{3} \mathrm{H}_{2}$ is assumed to be $2.5 \times 10^{-9}$. [2] Peak optical depths are $>0.7$ in CS absorption. [3] Appears to be a wing associated with the -73 or $-40 \mathrm{~km} \mathrm{~s}^{-1}$ gas instead of a separate component.

\subsubsection{CS-bearing molecules, $\mathrm{SO}$, and $\mathrm{H}_{2} \mathrm{CO}$}

$\mathrm{H}_{2} \mathrm{CO}$, SO, and most of the CS-bearing species share similar abundance patterns. The CS abundance varies by an order of magnitude within the clouds, and no trend is evident with the cloud hydrogen column. While an abundance of $\sim 1.6 \times 10^{-8}$ is most commonly measured, we note anomolously lower values of 2 to $6 \times 10^{-9}$ in some clouds, and a particularly high value of $2.5 \times 10^{-8}$ in the $-40 \mathrm{~km} \mathrm{~s}^{-1}$ cloud. In addition to CS, high abundances of $\mathrm{H}_{2} \mathrm{CO}, \mathrm{SO}, \mathrm{CCS}$, and $\mathrm{H}_{2} \mathrm{CS}$ are measured in the $-40 \mathrm{~km} \mathrm{~s}^{-1}$ cloud. Conversely, the abundance of $\mathrm{HCS}^{+}$is lower in the $-40 \mathrm{~km} \mathrm{~s}^{-1}$ cloud than in other measured clouds. Whereas $\mathrm{HCS}^{+}$is measured to have an abundance of $\sim 2.5$ to $3 \times 10^{-10}$ in most other clouds, it is somewhat lower at $1.4 \times 10^{-10}$ in the $-40 \mathrm{~km} \mathrm{~s}^{-1}$ cloud. Figure 10 shows the abundances of $\mathrm{H}_{2} \mathrm{CO}$, $\mathrm{SO}, \mathrm{CCS}, \mathrm{H}_{2} \mathrm{CS}$, and $\mathrm{HCS}^{+}$plotted against the abundance of $\mathrm{CS}$. The abundances of CCS and $\mathrm{H}_{2} \mathrm{CS}$ positively correlate with the CS abundance, although scatter exists. On the other hand, the abundance of $\mathrm{HCS}^{+}$does not vary linearly with the CS abundance; instead it is low in the $-40 \mathrm{~km} \mathrm{~s}^{-1}$ cloud and nearly constant for all other clouds. 


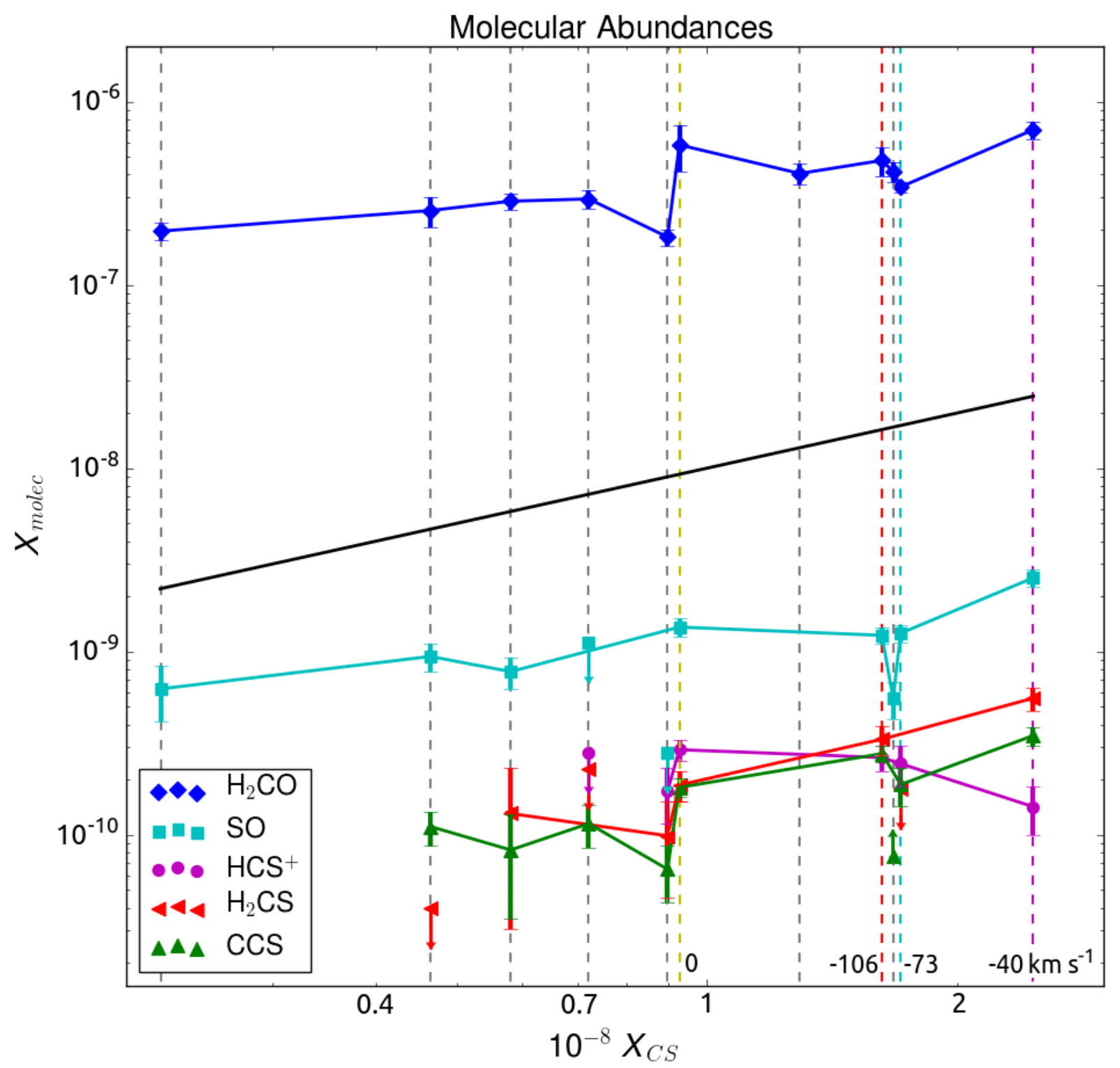

Fig. 10. Molecular abundances of $\mathrm{H}_{2} \mathrm{CO}, \mathrm{SO}$, and CS-bearing species plotted against the abundance of CS. The black solid line represents the identity line of the CS abundance. In order from lowest to highest CS abundance, the $-58,+20,-47,-23,-92, \sim 0,-120,-106,-80,-73$, and $-40 \mathrm{~km} \mathrm{~s}^{-1}$ components are marked with vertical dashed lines. Colored vertical lines, as labeled, mark the $-106,-73,-40$, and $0 \mathrm{~km} \mathrm{~s} \mathrm{~s}^{-1} \mathrm{clouds}$.

The abundances of $\mathrm{H}_{2} \mathrm{CO}$ and $\mathrm{SO}$ also positively correlate with that of CS. The observed trends in $\log \left(X_{\mathrm{SO}}\right)$ vs. $\log \left(X_{\mathrm{CS}}\right)$ and $\log \left(X_{\mathrm{H}_{2} \mathrm{CO}}\right)$ vs. $\log \left(X_{\mathrm{CS}}\right)$ are shallower than the CS-identity line, however, with slopes of $\sim 0.45$. Additionally, while these species show a clear correlation, statistically significant scatter is present.

\subsection{3. $\mathrm{SiO}$}

The abundance of $\mathrm{SiO}$ varies by an order of magnitude in different clouds. Furthermore, $X_{\mathrm{SiO}}$ does not correlate with $N_{\mathrm{H}_{2}}$ or $X_{\mathrm{CS}}$. In the $-73 \mathrm{~km} \mathrm{~s}^{-1}, 0 \mathrm{~km} \mathrm{~s}^{-1}$, and $+20 \mathrm{~km} \mathrm{~s}^{-1}$ clouds in the Galactic center, the $\mathrm{SiO}$ abundance is 1.3 to $2 \times 10^{-9}$. However, not all clouds believed to occur in the Galactic bar or Galactic center have high abundances of $\mathrm{SiO}$; in the $-58 \mathrm{~km} \mathrm{~s}^{-1}$ cloud, for example, $\mathrm{SiO}$ is at least an order of magnitude less abundant. In material located external to the Galactic bar at -23 and $-40 \mathrm{~km} \mathrm{~s}^{-1}, \mathrm{SiO}$ abundances are of order $4 \times 10^{-10}$.

\subsection{4. $l-\mathrm{C}_{3} \mathrm{H}$ and $l-\mathrm{C}_{3} \mathrm{H}^{+}$}

The abundances of $l-\mathrm{C}_{3} \mathrm{H}$ range from $\sim 1.5$ to $\sim 5 \times 10^{-10}$, with a median value of $2.2 \times 10^{-10}$, whereas the abundance of $l-\mathrm{C}_{3} \mathrm{H}^{+}$ varies from $4 \times 10^{-11}$ to $11 \times 10^{-10}$. The $l-\mathrm{C}_{3} \mathrm{H}$ and $l-\mathrm{C}_{3} \mathrm{H}^{+}$ abundances do not appear to correlate with $N_{\mathrm{H}_{2}}$ or with $X_{\mathrm{CS}}$.

\subsubsection{Isotopologue ratios}

The abundance ratios (or equivalently, integrated column density ratios) between isotopologues show significant variation within different clouds (Table 5 and Fig. 11). The ${ }^{12} \mathrm{C} /{ }^{13} \mathrm{C}$ ratio is probed by $\mathrm{H}_{2} \mathrm{CO}$ and $c-\mathrm{C}_{3} \mathrm{H}_{2}$. Values of $\mathrm{H}_{2} \mathrm{CO} / \mathrm{H}_{2}^{13} \mathrm{CO}$ range from 45 to 70 for the $-106,-40,-23$, and $\sim 0 \mathrm{~km} \mathrm{~s}^{-1}$ clouds, while higher values of $\sim 95$ are found in the material at -73 and $-80 \mathrm{~km} \mathrm{~s}^{-1}$. Thus, the ratios of $\mathrm{H}_{2} \mathrm{CO} / \mathrm{H}_{2}^{13} \mathrm{CO}$ do not show any obvious pattern with Galactocentric distance. Values of $c-\mathrm{C}_{3} \mathrm{H}_{2} / c-\mathrm{H}^{13} \mathrm{CCCH}$ are $\sim 25$ in the Galactic center clouds at 


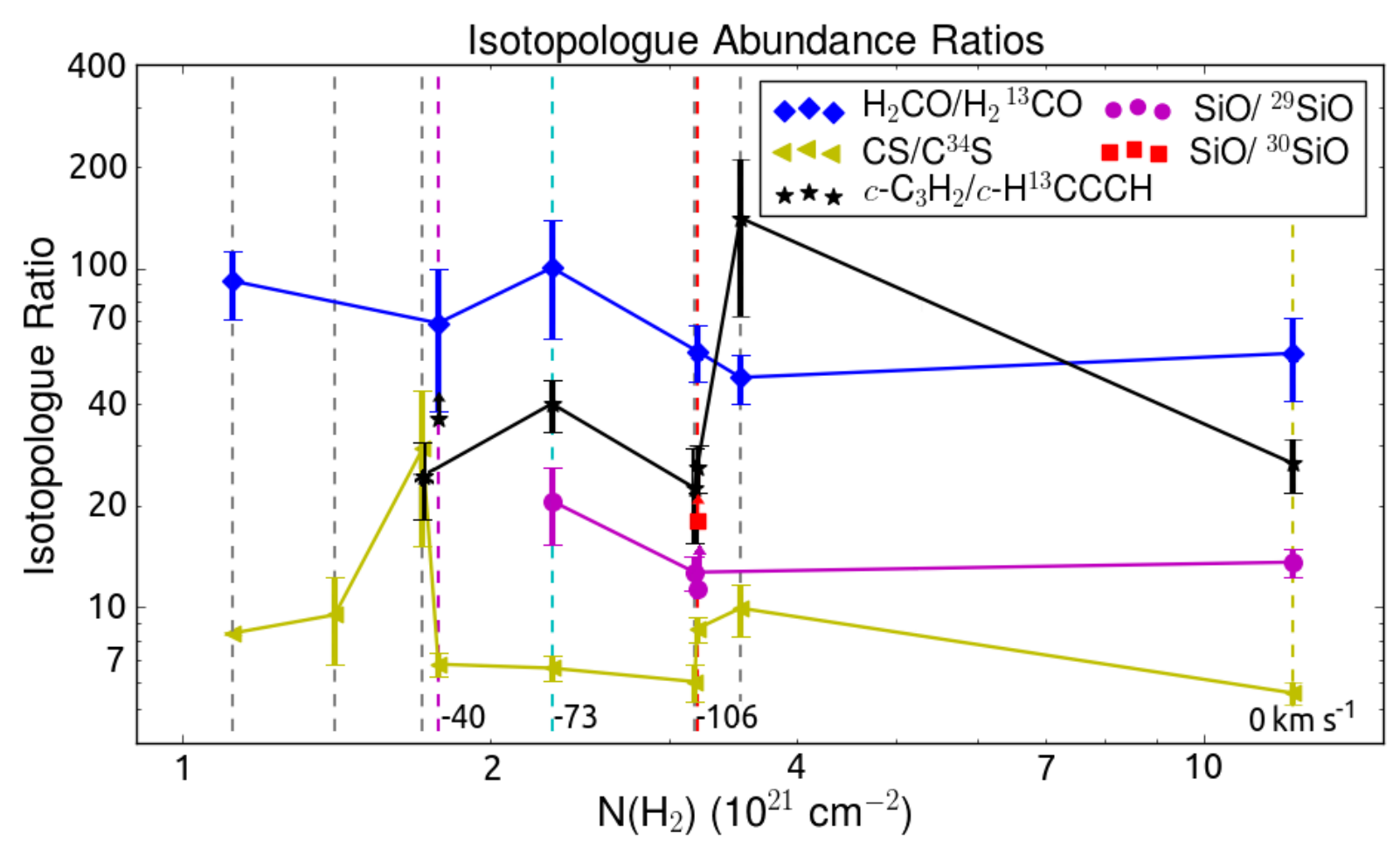

Fig. 11. Isotopologue abundance ratios. In order from lowest to highest hydrogen column, the $-80,-47,-92,-40,-73,+20,-106,-23$, and $\sim 0 \mathrm{~km} \mathrm{~s}^{-1}$ components are marked with vertical dashed lines. Colored vertical lines, as labeled, mark the $-106,-73,-40$, and $0 \mathrm{~km} \mathrm{~s}{ }^{-1} \mathrm{clouds}$.

Table 5. Isotopologue abundance ratios.

\begin{tabular}{lrrrrrrrrr}
\hline \hline Molecules & \multicolumn{7}{c}{ Absorption cloud velocity $\left(\mathrm{km} \mathrm{s}^{-1}\right)$} \\
& -106 & -92 & -80 & -73 & -47 & -40 & -23 & 0 & +20 \\
\hline$X\left(c-\mathrm{C}_{3} \mathrm{H}_{2}\right) / X\left(c-\mathrm{H}^{13} \mathrm{CCCH}\right)$ & $26(4)$ & $24(6)$ & & $40(7)$ & & $>36$ & $141(70)$ & $27(5)$ & $23(7)$ \\
$X\left(\mathrm{H}_{2} \mathrm{CO}\right) / X\left(\mathrm{H}_{2}^{13} \mathrm{CO}\right)$ & $57(10)$ & & $91(21)$ & $100(38)$ & & $69(31)$ & $48(8)$ & $56(15)$ & \\
$X(\mathrm{CS}) / X\left(\mathrm{C}^{34} \mathrm{~S}\right)$ & $8.7(8)^{[1]}$ & $29(14)$ & $8.4(1)$ & $6.6(6)^{[1]}$ & $10(3)$ & $6.8(5)^{[1]}$ & $10(2)$ & $5.6(4)^{[1]}$ & $6.0(7)$ \\
$X(\mathrm{SiO}) / X\left({ }^{29} \mathrm{SiO}\right)$ & $\gtrsim 11$ & & & $21(5)^{[2]}$ & & & & $13(1)$ & $13(2)$ \\
$\left.X(\mathrm{SiO}) / X{ }^{30} \mathrm{SiO}\right)$ & 218 & & & & & & & & \\
$X\left({ }^{29} \mathrm{SiO}\right) / X\left({ }^{30} \mathrm{SiO}\right)$ & $1.6(2)$ & & & & & & & & \\
\hline
\end{tabular}

Notes. [1] Peak CS absorption has a high optical depth of $\tau_{0}>0.7$. [2] Absorption in the velocity range of -85 to $-67 \mathrm{~km} \mathrm{~s}^{-1}$ was included in this measurement.

$-106,-92, \sim 0$, and $+20 \mathrm{~km} \mathrm{~s}^{-1}$, slightly higher at $\sim 40$ in the $-73 \mathrm{~km} \mathrm{~s}^{-1}$ cloud located deep within the Galactic bar, and significantly higher, at $\gtrsim 100$ in material in the Galactic disk at $-23 \mathrm{~km} \mathrm{~s}^{-1}$.

Isotope ratios of silicon are probed by measurements of ${ }^{28} \mathrm{Si}$, ${ }^{29} \mathrm{Si}$, and ${ }^{30} \mathrm{Si}$. In the $\sim 0$ and $+25 \mathrm{~km} \mathrm{~s}^{-1}$ material, we obtain ${ }^{28} \mathrm{SiO} /{ }^{29} \mathrm{SiO}=13$, and in the $-73 \mathrm{~km} \mathrm{~s}^{-1}$ cloud, we obtain a higher value of 21 . Due to the presence of an interloping line near $-106 \mathrm{~km} \mathrm{~s}^{-1}$ in the spectrum of ${ }^{28} \mathrm{SiO}$, we are unable to measure the ${ }^{28} \mathrm{SiO} /{ }^{29} \mathrm{SiO}$ ratio in that cloud. The ${ }^{29} \mathrm{Si} /{ }^{30} \mathrm{Si}$ ratio is measured to be 1.6 in the $-106 \mathrm{~km} \mathrm{~s}^{-1}$ cloud.

The ${ }^{32} \mathrm{~S} /{ }^{34} \mathrm{~S}$ ratio is probed by $\mathrm{CS} / \mathrm{C}^{34} \mathrm{~S}$. For all clouds except for the $-92 \mathrm{~km} \mathrm{~s}^{-1}$ cloud, the measured isotopologue ratio ranges from 5 to 10 . No trend is evident with hydrogen column or with Galactocentric distance. Within the components with high optical depths of peak $\tau>0.7$ in CS absorption, in which the measured isotopologue ratio may be depressed from the true value, we obtain measurements ranging from 5.6 to 8.7. In components with lower optical depths (of peak $\tau<0.5$ ) in the CS (1-0) line, values of 6 to $\sim 10$ are observed. In the $-92 \mathrm{~km} \mathrm{~s}^{-1}$ component, we obtain $\mathrm{CS} / \mathrm{C}^{34} \mathrm{~S}=29 \pm 14$, and it is unclear why this component is anomolous.

\section{Cloud hydrogen columns: considerations for interpreting abundances}

We have obtained new measurements of the molecular hydrogen columns within each cloud in the line-of-sight to Sgr B2, and of the abundances of 11 molecules and their isotopologues. Before discussing the implications of these measurements, we explore the validity of using $c-\mathrm{C}_{3} \mathrm{H}_{2}$ as a proxy for $\mathrm{H}_{2}$ and other preliminaries required for intrepreting the abundance measurements.

\subsection{Molecular hydrogen columns by conversion from $c-\mathrm{C}_{3} \mathrm{H}_{2}$}

In order to maximize the self consistency in our measurements, we probed the molecular hydrogen column using a molecule that is observed within our dataset; we selected $c-\mathrm{C}_{3} \mathrm{H}_{2}$ as the best proxy for $\mathrm{H}_{2}$ observable at centimeter wavelengths. In millimeter wavelength studies of diffuse and translucent clouds, hydrogen 
columns have typically been estimated by assuming a constant abundance of either $\mathrm{CO}$ or $\mathrm{HCO}^{+}$(e.g., Irvine et al. 1987; Greaves \& Nyman 1996). To the best of our knowledge, molecular hydrogen columns within diffuse and translucent clouds have not previously been estimated by conversion from $N_{c-\mathrm{C}_{3} \mathrm{H}_{2}}$. However, we suggest that the assumption of a constant abundance of $c-\mathrm{C}_{3} \mathrm{H}_{2}$ is reasonable and in fact may provide a more direct and accurate method of estimating the molecular hydrogen column than conversion from $\mathrm{CO}$ or $\mathrm{HCO}^{+}$.

Based on observations from radio to $\mathrm{UV}$ frequencies, the hydrocarbon molecules $\mathrm{CH}, \mathrm{CCH}$, and $c-\mathrm{C}_{3} \mathrm{H}_{2}$ appear to be constant in abundance with respect to one another and with respect to $\mathrm{H}_{2}$ in diffuse and translucent gas. The constancy of $N_{\mathrm{CH}} / N_{\mathrm{H}_{2}}$ over the range of $19<\log \left(N_{\mathrm{H}_{2}}\right)<22$ has been robustly demonstrated and directly calibrated by Sheffer et al. (2008) in an analysis of 90 sightlines with $\mathrm{CH}$ absorption and $\mathrm{H}_{2}$ Lyman-band observations. This hydrogen column range completely spans the domain of interest, from diffuse to the transition to dark molecular gas. $N_{\mathrm{CCH}} / N_{\mathrm{CH}}$ has also been shown to be constant in an analysis of Herschel HIFI data toward three sightlines that each contain multiple distinct diffuse and translucent clouds (Gerin et al. 2010b). The very tight correlation of $\tau_{\mathrm{CCH}}$ vs. $\tau_{\mathrm{CH}}$ was demonstrated over hundreds of channels in each sightline. Finally, multiple studies substantiate the constancy of $N_{c-\mathrm{C}_{3} \mathrm{H}_{2}} / N_{\mathrm{CCH}}$ in diffuse and translucent gas based on analyses of 26 distinct clouds in 9 sightlines (Lucas \& Liszt 2000; Gerin et al. 2011; Liszt et al. 2012). In these sightlines, $\mathrm{CCH}$ and $c-\mathrm{C}_{3} \mathrm{H}_{2}$ spectra show remarkable correlation, with a consistent opacity ratio present in channels from negligible optical depths to peak values (Lucas \& Liszt 2000; Gerin et al. 2011), and at various Galactocentric distances from the Galactic center to the outer disk (Gerin et al. 2011). In addition to having a stable abundance in diffuse and translucent clouds, $c-\mathrm{C}_{3} \mathrm{H}_{2}$ is observed to have precisely the same abundance with respect to molecular hydrogen in the dense $\left(n \sim 10^{5} \mathrm{~cm}^{-3}\right)$ Horsehead PDR (Pety et al. 2005), indicating that the value of $N(c$ $\left.\mathrm{C}_{3} \mathrm{H}_{2}\right) / N\left(\mathrm{H}_{2}\right)$ may be highly stable in UV-irradiated gas over a wide range of temperatures and densities. Therefore, the hydrocarbon species $\mathrm{CH}, \mathrm{CCH}$, and $c-\mathrm{C}_{3} \mathrm{H}_{2}$ appear to be good tracers of $N_{\mathrm{H}_{2}}$, as their abundances do not systematically vary with $N_{\mathrm{H}_{2}}$ over the column range of diffuse and translucent clouds.

On the other hand, the assumption of a constant $\mathrm{CO}$ abundance is easily contested in diffuse and translucent gas, given a growing body of evidence suggesting the presence of $\mathrm{CO}$ dark molecular gas in UV-irradiated environments (see e.g., Wolfire et al. 2010), and evidence that $N_{\mathrm{CO}} / N_{\mathrm{H}_{2}}$ varies by a factor of $>100$ in diffuse and translucent clouds (Sonnentrucker et al. 2007). The work of Sheffer et al. (2008) further demonstrates the variability in the $\mathrm{CO}$ abundance in diffuse and translucent clouds, with a sample of more than 100 sightlines with direct measurements of $N_{\mathrm{H}_{2}}$ and $N_{\mathrm{CO}}$; the data reveal that $N_{\mathrm{CO}} / N_{\mathrm{H}_{2}}$ systematically varies with $N_{\mathrm{H}_{2}}$, with a steep slope of $\log \left(N_{\mathrm{CO}}\right) / \log \left(N_{\mathrm{H}_{2}}\right)=3.1(7)$ above $N_{\mathrm{H}_{2}}=20.4$. Further, his data reveal substantial scatter, by nearly two orders of magnitude for a given hydrogen column. $\mathrm{CO}$ is thus a very poor indicator of total gas column in diffuse and translucent clouds.

While $\mathrm{HCO}^{+}$abundance variation does not appear to be as substantial as for $\mathrm{CO}, \mathrm{HCO}^{+}: \mathrm{CH}$ and $\mathrm{HCO}^{+}: \mathrm{CCH}$ have been shown to have a non-linear dependence on $N_{\mathrm{H}_{2}}$ (Lucas \& Liszt 2000; Gerin et al. 2010b). Like CO, if $N_{\mathrm{CH}} / N_{\mathrm{H}_{2}}$ is adopted as constant, then the abundance of $\mathrm{HCO}^{+}$increases in higher column density systems.

Finally, conversions from $\mathrm{CO}$ and $\mathrm{HCO}^{+}$are further complicated by the fact that their rotational transitions tend to be optically thick. While the abundances of the ${ }^{12} \mathrm{C}$ isotopologues are assumed to be constant for the purpose of conversion to $N_{\mathrm{H}_{2}}$, the column densities of the ${ }^{12} \mathrm{C}$ isotopologues often must first be estimated by conversion from ${ }^{13} \mathrm{CO}$ or $\mathrm{H}^{13} \mathrm{CO}^{+}$. Previous authors have assumed differing ratios of ${ }^{12} \mathrm{C} /{ }^{13} \mathrm{C}$ isotopologues internal to the Galactic center, bar, and disk. This is dangerous for numerous reasons, including that some line-of-sight clouds have poorly constrained Galactocentric distances. As is clear from our observations of isotopologues (Fig. 11), significant variation occurs in the isotopologue ratios in different clouds, and for many molecules, these cannot be accounted for by the Galactocentric distances presumed. It is also well established that isotope fractionation occurs creating discrepancies between the true isotope ratios (i.e., ${ }^{12} \mathrm{C} /{ }^{13} \mathrm{C}$ ) and the isotopologue ratios (i.e., ${ }^{12} \mathrm{CO} /{ }^{13} \mathrm{CO}$ and $\left.\mathrm{H}^{12} \mathrm{CO}^{+} / \mathrm{H}^{13} \mathrm{CO}^{+}\right)$.

Conversion from $c-\mathrm{C}_{3} \mathrm{H}_{2}$ is more direct, as it utilizes optically thin transitions of a molecule that has been systematically explored in diffuse and translucent clouds over a broad range of cloud thicknesses and throughout the Galaxy (Lucas \& Liszt 2000; Gerin et al. 2011; Liszt et al. 2012) and does not require an isotope ratio conversion. As such, $c-\mathrm{C}_{3} \mathrm{H}_{2}$ appears to be the best means of determining the hydrogen column at our disposal, and we recommend further exploration and precise calibration of the $c-\mathrm{C}_{3} \mathrm{H}_{2}$ abundance via observations and modeling. Despite the various issues with using $\mathrm{CO}$ and $\mathrm{HCO}^{+}$, we note general agreement between our measured values of $\mathrm{N}_{\mathrm{H}_{2}}$ and previous measurements of the same clouds in the line-of-sight to Sgr B2(M) located $\sim 45$ arcsec south (Table 3 ).

\subsection{Defining Cloud Borders}

We also point out differences in how we distinguished between clouds. In the previous studies, authors have combined multiple cloud components for hydrogen column and molecular abundance estimates. For example, the clouds at -47 and $-40 \mathrm{~km} \mathrm{~s}^{-1}$ were treated as a single cloud in analyses by previous authors (Irvine et al. 1987; Greaves \& Nyman 1996; Wirström et al. 2010). As the -47 and $-40 \mathrm{~km} \mathrm{~s}^{-1}$ clouds have demonstrable differences in molecular abundances, for example in the abundance of CS, we treated these systems as distinct. For the purpose of follow-up studies of the chemistry within these environments, we consider it prudent to treat the clouds as specifically as is possible rather than present average abundance values which may not be representative of any environment in the cloud. In this vein, we might consider dividing the clouds further. For example, the $-40 \mathrm{~km} \mathrm{~s}^{-1}$ cloud contains two narrow velocity components that are clearly resolvable in CS absorption. However, for many of the lower $\mathrm{S} / \mathrm{N}$ transitions, we do not have the $\mathrm{S} / \mathrm{N}$ required to distinguish the two components. In this manner, we have maximized the specificity with which we may treat individual clouds.

Finally, it is standard to interpret a hydrogen column as an extinction value by Eq. (5). Obviously, the implied extinction values will vary depending on where the clouds are distinguished. For example, if the -47 and $-40 \mathrm{~km} \mathrm{~s}^{-1}$ clouds are adjacent and do not contain UV-radiation sources located between the two clouds, then it is, indeed, physically appropriate to treat the two clouds as a combined system with a higher extinction. However, we know little about the three-dimensional geometry of the clouds and incident radiative fields. In fact, substantial differences may occur in the incident UV radiation field depending on the Galactocentric distance and on the precise local environment of the cloud, so that two clouds with similar hydrogen columns may experience very different radiative fields at 
their centers. Secondly, cosmic rays, known to be multiple orders of magnitude more prevalent in diffuse clouds the Galactic center (Le Petit et al. 2016), may induce local UV-radiation fields very deep within the cloud, and X-rays which are particularly prevalent in the Galactic center can penetrate the diffuse and translucent clouds (Amo-Baladrón et al. 2009). Finally, the geometry and structure of the diffuse and translucent ISM can significantly affect the radiative field experienced within the cloud. Thus, we do not put much emphasis on the total hydrogen column as a determinant of the chemistry observed within the clouds, and indeed we do not observe significant trends with total hydrogen column in Figs. 8 and 9. Instead, we point out differences between clouds located internal and external to the Galactic center and Galactic bar, and look for clues to the structure of different clouds.

Translucent clouds have typical central extinctions of $1<A_{\mathrm{V} 0}<2.5$. Many of the clouds, as divided in this work, do not meet this threshold, having central extinction values of 0.5 to 1.0 (Table 3 ). This clearly depends on how the clouds are binned, however we point out that many of the clouds considered may be intermediate between diffuse clouds and translucent clouds as defined by this extinction threshold, and are likely transitional regions. This result deserves further exploration however, as the average molecular hydrogen fractions, estimated at 0.65 to 0.95 , are significantly higher than the values of 0.4 to 0.5 commonly observed in diffuse gas (Liszt et al. 2012; Snow \& McCall 2006). Nonetheless, it may be reasonable to consider many of the these systems as diffuse or transitional clouds based on the dominant form of carbon in the material. The classification system for diffuse atomic, translucent, and dark clouds introduced by Snow \& McCall (2006) defines translucent clouds based on the dominant form of carbon, as having $f\left(\mathrm{C}^{+}\right)<0.5$ and $f(\mathrm{CO})<0.9$. Studies of OI, CII, and ${ }^{13} \mathrm{CO}$ line profiles toward Sgr B2(N) show that carbon is predominantly in the neutral and ionized forms within this material (Vastel et al. 2002), indicating that it may indeed be transitional between diffuse molecular and translucent.

\section{Discussion}

We now discuss the implications of the results described in Sect. 3 for the locations and physical structure of the line-ofsight clouds.

\subsection{Discussion of isotopologue ratios and fractionation}

The observed isotopologue ratios of $\mathrm{H}_{2} \mathrm{CO}$ and $c-\mathrm{C}_{3} \mathrm{H}_{2}$ suggest systematic differences between the two. Therefore, it is clear that isotope fractionation is present for at least one of the species. While isotope ratios of $c-\mathrm{C}_{3} \mathrm{H}_{2}$ have not been studied closely, models by Langer et al. (1984) indicate fractionation occurs for the $\mathrm{H}_{2} \mathrm{CO} / \mathrm{H}_{2}^{13} \mathrm{CO}$ ratio, such that this provides an upper limit to ${ }^{12} \mathrm{C} /{ }^{13} \mathrm{C}$, while the ${ }^{12} \mathrm{CO} /{ }^{13} \mathrm{CO}$ ratio provides a lower limit. Extensive studies of the ${ }^{12} \mathrm{C} /{ }^{13} \mathrm{C}$ ratio have been published using isotopologues of $\mathrm{CO}, \mathrm{H}_{2} \mathrm{CO}, \mathrm{CN}$, and $\mathrm{CH}^{+}$(Wilson 1999; Wilson \& Rood 1994; Stahl et al. 2008; Milam et al. 2005). While the studies show definitive evidence for variation in different sources of the same galactocentric distance, a general trend of increasing ${ }^{12} \mathrm{C} /{ }^{13} \mathrm{C}$ ratios with increasing Galactocentric distance $D_{\mathrm{GC}}$ is well established, with typical values of $\sim 20$ in the Galactic center, $\sim 50$ at a Galactocentric distance of $4 \mathrm{kpc}$, and $\sim 70$ at the solar Galactocentric distance. In most clouds located internal to the Galactic bar, the $\mathrm{H}_{2} \mathrm{CO} / \mathrm{H}_{2}^{13} \mathrm{CO}$ ratios measured are a factor of two to three higher than expected, and the reported values do not show a clear trend with Galactocentric distance, but do show statistically significant variation. The ratios measured by $c-\mathrm{C}_{3} \mathrm{H}_{2} / c-\mathrm{H}^{13} \mathrm{CCCH}$, namely $\sim 25$ in the Galactic center, 40 at $\lesssim 1 \mathrm{kpc},>36$ at $3 \mathrm{kpc}$, and $\gtrsim 100$ at $4 \mathrm{kpc}$, show a very clear trend with Galactocentric distance, and are very similar to the previous measurements of ${ }^{12} \mathrm{C} /{ }^{13} \mathrm{C}$ ratios.

The isotope ratio of ${ }^{32} \mathrm{~S} /{ }^{34} \mathrm{~S}$ has also been investigated (Frerking et al. 1980; Chin et al. 1996; Mauersberger et al. 1996), but it is not as thoroughly researched as the carbon isotope ratio, and disagreement persists over what trends, if any, are present with Galactocentric distance. Previous studies typically obtained values of $24.4 \pm 5$, whereas, we observe substantially lower values of $5<\mathrm{CS} / \mathrm{C}^{34} \mathrm{~S}<10$ for all clouds with the exception of the $-92 \mathrm{~km} \mathrm{~s}^{-1}$ cloud, which has a higher value of $29 \pm 15$. The measured ratios do not show any apparent trend with Galactocentric distance. Generally, chemical fractionation is not believed to affect sulfur chemistry substantially, as the zero point energies of ${ }^{32} \mathrm{~S}$ and ${ }^{34} \mathrm{~S}$ are quite similar.

Finally, the ratios of ${ }^{28} \mathrm{Si} /{ }^{29} \mathrm{Si}$ and ${ }^{29} \mathrm{Si} /{ }^{30} \mathrm{Si}$ have not been systematically investigated in the Galaxy. In the $\sim 0$ and $+25 \mathrm{~km} \mathrm{~s}^{-1}$ material, we obtain ${ }^{28} \mathrm{SiO} /{ }^{29} \mathrm{SiO}=13$, and in the $-73 \mathrm{~km} \mathrm{~s}^{-1}$ cloud, we obtain a higher value of $21 \pm 5$. Additionally, we measure ${ }^{29} \mathrm{SiO} /{ }^{30} \mathrm{SiO}=1.6 \pm 2$. Within the solar system, a value of ${ }^{28} \mathrm{Si} /{ }^{29} \mathrm{Si}=19.7$ is observed (Wilson 1999 ; Penzias 1981). Assuming that chemical fractionation does not significantly affect the $\mathrm{SiO}$ isotopoluges, our data indicates that the ${ }^{28} \mathrm{Si} /{ }^{29} \mathrm{Si}$ in the $-73 \mathrm{~km} \mathrm{~s}^{-1}$ cloud is consistent with the local value, while ${ }^{29} \mathrm{Si}$ is enhanced relative to the main isotope in the $\sim 0$ and $+25 \mathrm{~km} \mathrm{~s}^{-1}$ material located in the Galactic center. Additionally, early investigations of ${ }^{29} \mathrm{Si} /{ }^{30} \mathrm{Si}$ have determined values of 1.5 without a gradient with galactocentric distance (Penzias 1981; Huettemeister et al. 1998). The measured value of ${ }^{29} \mathrm{Si} /{ }^{30} \mathrm{Si}$ in the $-106 \mathrm{~km} \mathrm{~s}^{-1}$ gas is consistent with this, supporting the supposition that the value of ${ }^{29} \mathrm{Si} /{ }^{30} \mathrm{Si}$ does not systematically vary with Galactocentric distance.

Given these observations, a few trends can be pointed out. First, $\mathrm{H}_{2} \mathrm{CO} / \mathrm{H}_{2}^{13} \mathrm{CO}$ is not a good probe of the ${ }^{12} \mathrm{C} /{ }^{13} \mathrm{C}$ ratio, and includes significant fractionation. If the fractionation can be understood, this may be interesting from a chemistry perspective. Secondly, the values of $c-\mathrm{C}_{3} \mathrm{H}_{2} / c-\mathrm{H}^{13} \mathrm{CCCH}$ exhibit a trend with Galactocentric distance, and are consistent with previous measurements of ${ }^{12} \mathrm{C} /{ }^{13} \mathrm{C}$ ratios. This suggests that isotope fractionation is not significant for these two isotopologues of $c-\mathrm{C}_{3} \mathrm{H}_{2}$, so that $c-\mathrm{C}_{3} \mathrm{H}_{2}$ can probe the ${ }^{12} \mathrm{C} /{ }^{13} \mathrm{C}$ ratio accurately, with the added advantage over $\mathrm{CO}$ and $\mathrm{HCO}^{+}$of being optically thin. Third, ${ }^{34} \mathrm{~S}$ is substantially enhanced in the clouds measured here compared to previous measurements in other clouds, assuming that $\mathrm{CS} / \mathrm{C}^{34} \mathrm{~S}$ does not undergo fractionation. The PRIMOS data support the supposition that there is no gradient in ${ }^{32} \mathrm{~S} /{ }^{34} \mathrm{~S}$ with Galactocentric distance.

Finally, the isotopologue ratios provide clues as to the locations of the line-of-sight clouds in the Galaxy. For example, the $-73 \mathrm{~km} \mathrm{~s}^{-1}$ cloud has values of $c-\mathrm{C}_{3} \mathrm{H}_{2} / c-\mathrm{H}^{13} \mathrm{CCCH}$ that are intermediate between those observed in Galactic center clouds and those of clouds located in to the Galactic disk and previously measured locally; further, the ${ }^{28} \mathrm{SiO} /{ }^{29} \mathrm{SiO}$ ratio in the $-73 \mathrm{~km} \mathrm{~s}^{-1}$ cloud is clearly distinct from the values measured in the Galactic center clouds and is comparable to the value obtained in the solar system. These observations are consistent with the $-73 \mathrm{~km} \mathrm{~s}^{-1}$ material being located external to the Galactic center and in the bar. The observations therefore lend further support to the approximate locations of the clouds in the Galaxy. 


\subsection{Sulfur-bearing chemistry: $\mathrm{CS}, \mathrm{SO}, \mathrm{HCS}^{+}, \mathrm{CCS}$, and $\mathrm{H}_{2} \mathrm{CS}$}

Clearly, diverse sulfur-bearing chemistry is observed within the diffuse and translucent clouds. The abundances of a few of the sulfur-bearing molecules detected here have been investigated in previous studies of diffuse and translucent clouds, most recently by Lucas \& Liszt (2002) and Neufeld et al. (2015). Lucas \& Liszt (2002) measured the abundances of CS, SO, and $\mathrm{HCS}^{+}$in diffuse clouds absorbing against extragalactic point sources at high Galactic latitude, while Neufeld et al. (2015) measured the abundances of CS and SO in diffuse and translucent clouds in the Galactic plane. In this section, we discuss our observational results in frequent reference to these two papers. Additionally, Drdla et al. (1989) reported abundance measurements of CS and $\mathrm{HCS}^{+}$in diffuse and/or translucent clouds at high Galactic latitude, and contributed the most comprehensive theoretical model predictions for sulfur-bearing molecule abundances in diffuse clouds published to date.

In the following discussion, we come to the following main conclusions on sulfur-bearing chemistry based on the data shown here and in the context of the three forementioned papers. First, in clouds in the Galactic disk, the data suggests that CS, and likely SO to a lesser degree, preferentially inhabit the highest density regions of the diffuse and translucent clouds, with abundance enhancements in high density material. Secondly, whereas the abundances of CS and $\mathrm{HCS}^{+}$are predicted to be closesly coupled (Drdla et al. 1989; Lucas \& Liszt 2002), we do not observe this and our abundance ratios of $\mathrm{CS}: \mathrm{HCS}^{+}$disagree with the observations of Lucas \& Liszt (2002) and Neufeld et al. (2015). Third, $\mathrm{SO}$ and $\mathrm{H}_{2} \mathrm{CS}$ are severely overabundant as compared to model predictions (Drdla et al. 1989), and model predictions for CCS in diffuse and translucent clouds have not been published. With the wealth of data published, and with recent advances in chemical models, we suggest the sulfur-bearing chemistry in diffuse clouds should be revisited.

\subsubsection{CS: abundances and implications for cloud structure}

Our reported CS abundances vary by more than an order of magnitude, with a few clouds having values in the range of 2 to $7 \times 10^{-9}$ and multiple clouds in the range of 1 to $2.5 \times 10^{-8}$. The latter set of clouds have high abundances of CS as compared to most interstellar environments, with values similar to the abundances observed in the hot core in Sgr B2(N) and in the Orion Molecular Ridge (Neill et al. 2014; Crockett et al. 2014) and a factor of two to three times higher than in the dense Horsehead PDR (Goicoechea et al. 2006) and the dark cloud TMC-1 (Ohishi et al. 1992). The measurements presented here are in approximate agreement with values reported by Greaves \& Nyman (1996) in the line-of-sight to Sgr B2(M), of 2 to $5 \times 10^{-8}$. However, the CS abundances measured in most of the clouds in the sightlines to Sgr B2(N) and (M) are significantly larger than those reported in diffuse clouds toward other sightlines by Lucas \& Liszt (2002) and Neufeld et al. (2015). In both latter papers, the authors derived abundances that typically ranged from 1 to $5 \times 10^{-9}$, although one of the ten clouds measured by Neufeld et al. (2015) was larger, at $10^{-8}$. While Lucas \& Liszt (2002) typically obtained lower values than we measure, they noted that the CS abundance appears sporadic in the diffuse clouds; in some clouds with significant column densities of $\mathrm{HCO}^{+}$, and presumably therefore of $\mathrm{H}_{2}, \mathrm{CS}$ was undetected, whereas it was clearly detected in other clouds with lower $\mathrm{HCO}^{+}$columns. In the data presented here, by Lucas \& Liszt (2002), and by Neufeld et al. (2015), the abundance of CS does not appear to scale with the total molecular or neutral hydrogen column. Additionally, we do not observe systematically higher or lower abundances in the Galactic center or bar clouds compared to disk clouds.

While there is no clear differentiation between Galactic center or bar clouds and disk clouds based on the CS abundance values observed, there is an apparent distinction between the shapes of the CS profiles in Galactic center clouds compared to disk clouds. As described in Sect. 3.1 the CS absorption in Galactic center and bar clouds, for example at $-106,-73$, and $0 \mathrm{~km} \mathrm{~s}^{-1}$, includes absorption over the same moderately broad velocity ranges as do $c-\mathrm{C}_{3} \mathrm{H}_{2}$ and $\mathrm{H}_{2} \mathrm{CO}$. On the other hand, the profile of CS contains very sharp, narrow peaks in the clouds located external to the Galactic bar, namely in the -47 and $-40 \mathrm{~km} \mathrm{~s}^{-1}$ clouds believed to be located in the $3-\mathrm{kpc}$ arm at the outer edge of the bar and in the $-23 \mathrm{~km} \mathrm{~s}^{-1}$ cloud. This suggests that CS is highly sensitive to the physical conditions within the clouds, and may indicate a systematic difference in the structure of clouds that are located in the Galactic center compared to those present in the disk.

In the Galactic disk clouds, in which CS contains narrow peaks with little broad component absorption as compared to $c$ $\mathrm{C}_{3} \mathrm{H}_{2}$ and $\mathrm{H}_{2} \mathrm{CO}$, we suggest that $\mathrm{CS}$ is significantly more abundant in the highest density regions of the clouds. While chemical models notoriously underpredict abundances of sulfur-bearing species in the diffuse ISM, the known reactions can achieve higher CS abundances at higher physical densities and/or higher visual extinctions (see e.g., Lucas \& Liszt 2002; Neufeld et al. 2015, and references therein). However, as the total hydrogen column has been shown to be a poor predictor of the CS column density and abundance in the data presented here, in Lucas \& Liszt (2002), and in Neufeld et al. (2015), it appears that the CS abundance is more sensitive to density conditions than to extinction in the diffuse and translucent medium. This claim is also supported by previous observations; the work of Greaves et al. (1992) suggests that CS resides in material that is significantly more dense than average in the line-of-sight to Sgr B2(M). While the mean densities in the clouds appear to be $n \sim 300$ to $500 \mathrm{~cm}^{-3}$, CS resides in material with densities of $4000 \lesssim n \lesssim 15000 \mathrm{~cm}^{-3}$ as estimated by a Large Velocity Gradient (LVG) analysis of CS (1-0) and (2-1).

If $\mathrm{CS}$ is sensitive to density but not directly correlated with extinction, then this suggests that the density profile in the diffuse clouds is not a simple, smooth function of extinction. The CS profiles therefore suggest that the diffuse and translucent clouds in the disk have a clumpy physical structure, as the CS observations cannot be accounted for by a gradual density enhancement with $A_{\mathrm{V}}$. Furthermore, the differences between the $\mathrm{CS}$ profile and the profiles of most other molecules suggests that CS is preferentially residing in a different chemical environments. This in turn suggests a layered chemical structure, with a greater number of chemical environments than previously described.

\subsubsection{SO}

The absorption profile of SO suggests that it too has an enhanced abundance in the highest density regions in the -47 , -40 , and $-23 \mathrm{~km} \mathrm{~s}^{-1}$ clouds, with narrow peaks dominating the absorption in the -47 and $-40 \mathrm{~km} \mathrm{~s}^{-1}$ clouds especially. The abundance of SO shows a positive linear correlation with the CS abundance but does not vary as much as CS does (Fig. 10). Therefore the SO abundance may be enhanced, but by a lower factor than $\mathrm{CS}$, in the highest density regions. 
Lucas \& Liszt (2002) also found a positive but loose correlation between $\mathrm{SO}$ and CS in diffuse gas.

The SO abundances, with a median value of $1.1 \times 10^{-9}$ are similar to those measured by Lucas \& Liszt (2002) and Neufeld et al. (2015). Lucas \& Liszt (2002) notes that gas phase models of sulfur-bearing chemistry underpredict this value by multiple orders of magnitude. Even upon considering shocks and turbulent dissipation, models underpredict the SO abundance by an order of magnitude (Neufeld et al. 2015). Because the abundances of CS are significantly higher in most clouds along the sightline to Sgr B2(N), we obtain significantly larger values of the ratio $N_{\mathrm{CS}} / N_{\mathrm{SO}}$ than do Lucas \& Liszt (2002) and Neufeld et al. (2015). In most clouds, these authors found that $N_{\mathrm{CS}} / N_{\mathrm{SO}}=2$, although a higher value of 5.8 was present in the cloud with the anomolously high CS abundance, mentioned above, in Neufeld et al. (2015). We obtain values of $N_{\mathrm{CS}} / N_{\text {SO }}$ ranging from 3 to $\sim 30$, with a median value of 9 . In other Galactic interstellar environments, ratios of $N_{\mathrm{CS}} / N_{\mathrm{SO}}$ have been observed to vary significantly in different clouds; even in clouds in similar evolutionary states and with apparently similar conditions, very different values may be observed (e.g., Gerin et al. 1997).

\subsection{3. $\mathrm{HCS}^{+}, \mathrm{CCS}$, and $\mathrm{H}_{2} \mathrm{CS}$}

Among the lower abundance CS-bearing species, the results demonstrate a positive linear correlation between the abundances of $\mathrm{CCS}$ and $\mathrm{H}_{2} \mathrm{CS}$ with $\mathrm{CS}$ (Fig. 10), although scatter exists, due in part to baseline instability and line confusion in the lower signal transitions. On the other hand, $\mathrm{HCS}^{+}$displays a different abundance pattern, with a fairly consistent abundance in all clouds except for the $-40 \mathrm{~km} \mathrm{~s}^{-1}$ cloud. While observation of additional transitions of $\mathrm{HCS}^{+}$should be conducted to confirm this trend, the depressed abundance of $\mathrm{HCS}^{+}$in the $-40 \mathrm{~km} \mathrm{~s}^{-1}$ cloud is notable because all other sulfur-bearing species have higher abundances in this cloud than in any other. If a larger fraction of the $-40 \mathrm{~km} \mathrm{~s}^{-1}$ cloud consists of higher density material, it may be that the $\mathrm{HCS}^{+}$abundance is lower in the highest density regions of diffuse and translucent clouds. The abundance patterns thus suggest that the ratios of $N_{\mathrm{HCS}^{+}} / N_{\mathrm{CS}}$, $N_{\mathrm{HCS}^{+}} / N_{\mathrm{CCS}}$, and $N_{\mathrm{HCS}^{+}} / N_{\mathrm{H}_{2} \mathrm{CS}}$ appear to be sensitive to cloud conditions within diffuse and translucent clouds, making these ratios potentially useful probes of physical conditions. Although in a distinct environment, Corby et al. (2015) observed that CS, CCS, and $\mathrm{H}_{2} \mathrm{CS}$ have indistinguishable spatial distributions in Sgr B2, while $\mathrm{HCS}^{+}$has a distinct spatial distribution, further supporting the trend that $\mathrm{CS}, \mathrm{CCS}$, and $\mathrm{H}_{2} \mathrm{CS}$ track each other, and that the ratios of $\mathrm{HCS}^{+}$to other CS-bearing species are sensitive to physical conditions.

According to chemical models, the molecules $\mathrm{HCS}^{+}$and CS are among the most closely related species present in the ISM, as they participate in a direct exchange in both the formation and destruction of CS (Drdla et al. 1989; Lucas \& Liszt 2002). In the reaction network considered by Drdla et al. (1989) and Lucas \& Liszt (2002), CS is believed to be formed primarily from the dissociative recombination reaction of

$\mathrm{HCS}^{+}+\mathrm{e}^{-} \rightarrow \mathrm{CS}+\mathrm{H}$

where $\mathrm{HCS}^{+}$is first formed by reactions beginning with $\mathrm{S}^{+}$. However, recent experiments indicate that this product channel occurs in only 19 percent of collisions, with 81 percent of collisions forming $\mathrm{CH}+\mathrm{S}$ (Montaigne et al. 2005). Three mechanisms dominate the destruction of CS in these networks. In the first two, photoionization and ion-exchange reactions destroy CS to form $\mathrm{CS}^{+}$, by

$\mathrm{CS}+\gamma \rightarrow \mathrm{CS}^{+}+\mathrm{e}^{-}$,

and

$\mathrm{CS}+X^{+} \rightarrow \mathrm{CS}^{+}+X$

where $X^{+}$is a cationic species and $X$ is the corresponding neutral species. $\mathrm{CS}^{+}$then quickly reacts to form $\mathrm{HCS}^{+}$:

$\mathrm{CS}^{+}+\mathrm{H}_{2} \rightarrow \mathrm{HCS}^{+}+\mathrm{H}$.

In the third destruction route, $\mathrm{CS}$ reacts with $\mathrm{H}_{3}^{+}$to form $\mathrm{HCS}^{+}$directly:

$\mathrm{CS}+\mathrm{H}_{3}^{+} \rightarrow \mathrm{HCS}^{+}+\mathrm{H}_{2}$.

As CS is formed by a reaction of $\mathrm{HCS}^{+}$and the dominant destruction pathways for $\mathrm{CS}$ form $\mathrm{HCS}^{+}$, we would expect the abundances of $\mathrm{CS}$ and $\mathrm{HCS}^{+}$to be delicately balanced, and for the two abundances to track each other. While chemical models of sulfur-bearing chemistry in diffuse clouds underpredict the abundance of $\mathrm{HCS}^{+}$by multiple orders of magnitude, Lucas \& Liszt (2002) determined that if the observed abundance of $\mathrm{HCS}^{+}$is injected into a diffuse cloud, it is possible to account for the CS abundances observed in their sample, further emphasizing the theoretical importance of $N_{\mathrm{CS}} / N_{\mathrm{HCS}^{+}}$. In clouds in the Galactic center, where $\mathrm{H}_{3}^{+}$is $\gtrsim 10$ times more abundant compared to diffuse clouds in the disk (Oka et al. 2005), we might expect an offset in the ratio, tending toward a higher relative abundance of $\mathrm{HCS}^{+}$.

In stark contrast to this expectation, the abundance of $\mathrm{HCS}^{+}$appears to be uncorrelated with that of $\mathrm{CS} \mathrm{HCS}^{+}$exhibits a typical abundance of 2.5 to $3 \times 10^{-10}$ in this study, and this value closely matches the measurements made in the diffuse clouds sampled by Lucas \& Liszt (2002). On the other hand, we observe very large variations in CS abundances, by a factor of $\gtrsim 10$ in our study and $\gtrsim 25$ if we also consider the measurements made by Lucas \& Liszt (2002) and Neufeld et al. (2015). Furthermore, instead of observing more $\mathrm{HCS}^{+}$relative to $\mathrm{CS}$ in the Galactic center clouds due to the higher abundance of $\mathrm{H}_{3}^{+}$, we see the opposite. Whereas Lucas \& Liszt (2002) measured $N_{\mathrm{CS}} / N_{\mathrm{HCS}^{+}}=13.3 \pm 1.0$ in diffuse clouds in the Galactic disk, we find much higher values (and therefore lower $\mathrm{HCS}^{+}$abundances relative to $\mathrm{CS}$ ) ranging from 50 to 70 in most Galactic center and bar clouds. The constancy of the $\mathrm{HCS}^{+}$abundance in clouds measured in this study and in Lucas \& Liszt (2002) indicates that the $\mathrm{HCS}^{+}$abundance is (1) consistent over a wide range of densities and extinction values; (2) independent of the CS abundance; and (3) independent of the Galactocentric distance. As the same values are measured in absorbing clouds in the Galactic disk as in the Galactic center and bar, it appears that the $\mathrm{HCS}^{+}$abundance is not highly sensitive to the CR-ionization rate or X-ray fluxes in diffuse gas.

$\mathrm{CCS}$ and $\mathrm{H}_{2} \mathrm{CS}$ have similar abundances to $\mathrm{HCS}^{+}$, with typical values of 1 to $3 \times 10^{-10}$; like $\mathrm{HCS}^{+}, \mathrm{CCS}$, and $\mathrm{H}_{2} \mathrm{CS}$ are significantly more abundant than predicted by models appropriate in diffuse, translucent, and/or any other UV-irradiated material (e.g., Drdla et al. 1989; Goicoechea et al. 2006). In fact, the observed enhancement of $\mathrm{H}_{2} \mathrm{CS}$ is five orders of magnitude larger than predicted by Drdla et al. (1989), and no prediction was published for CCS. With the recent observational constraints on sulfur-bearing chemistry in diffuse and translucent clouds provided here, in Lucas \& Liszt (2002) and Neufeld et al. (2015), 
and in dense PDRs (Goicoechea et al. 2006), a revised theoretical treatment of sulfur-bearing chemistry, and particularly of CSbearing chemistry, is warranted. More advanced chemical models including PDR and gas-grain models with updated reaction rates for sulfur-bearing species are being developed; the application of these to physical conditions appropriate in diffuse and translucent clouds could prove effective for reproducing the observed chemistry. More importantly, a thorough theoretical treatment of the sulfur-bearing chemistry may illuminate the patterns embedded in the varying abundance ratios, producing a more nuanced understanding of what the chemistry tells us about the physical and radiative structure of the diffuse and translucent ISM. For example, the ratio of $N_{\mathrm{CS}}$ to $N_{\mathrm{HCS}^{+}}$may prove an excellent probe of physical conditions, however a solid theoretical understanding of the reaction networks is requisite for interpreting the ratios.

\subsection{Oxygen-bearing chemistry: $\mathrm{OH}, \mathrm{H}_{2} \mathrm{CO}$, and $\mathrm{SiO}$}

\subsubsection{Interpreting $\mathrm{OH}$ absorption}

$\mathrm{OH}$ has four hyperfine transitions in the $\left({ }^{2} \Pi_{3 / 2} J=\frac{3}{2}\right)$ state, at $1612,1665,1667$, and $1720 \mathrm{MHz}$. All four transitions can exhibit masing, typically in high density $\left(10^{6} \leq n \lesssim 10^{9} \mathrm{~cm}^{-3}\right)$ environments (Gray et al. 1991; Elitzur 1992) associated with star formation, shocks, and AGB winds. Under lower density conditions in which strong masing is not produced, thermal absorption or emission line profiles are observed. However, to the best of our knowledge, there have been no reported observations in which the four hyperfine transitions are in LTE. Instead, recent work by Ebisawa et al. (2015) has demonstrated that non-LTE excitation of the ground state hyperfine transitions of $\mathrm{OH}$ are ubiquitous in clouds that do not host strong masing, including a translucent cloud, a cold dark cloud, and a PDR, and additional reports of similar behaviors have been reported commonly (see references in Ebisawa et al. 2015), although the reported instances tend to involve emission line sources. In the study conducted by Ebisawa et al. (2015), the 1665 and $1667 \mathrm{MHz}$ transitions produce emission that is more consistent with LTE conditions over a wider range of physical conditions, while the $1612 \mathrm{MHz}$ component appears in absorption against the CMB background and enhanced emission is present in the $1720 \mathrm{MHz}$ line. The divergence from LTE occurs by a collisional excitation mechanism, even at extremely low physical densities of $\sim 10 \mathrm{~cm}^{-3}$ (Elitzur 1992).

In Sect. 3.5.1, we described that the $1665 \mathrm{MHz}$ and $1667 \mathrm{MHz}$ transitions have very consistent absorption profiles, with primarily broad $\left(\Delta v \geq 10 \mathrm{~km} \mathrm{~s}^{-1}\right)$ absorption components, resulting in statistically identical $\mathrm{OH}$ column density measurements. However, the profile of the $1612 \mathrm{MHz}$ transition is clearly different, as are the column density values measured using the $1612 \mathrm{MHz}$ transition. The discrepancy between the measured values indicates that a non-LTE excitation effect is present for $\mathrm{OH}$ in diffuse clouds. The 1665 and $1667 \mathrm{MHz}$ transitions should better represent the true kinematic distribution and column density of $\mathrm{OH}$, and we thus exclude the $1612 \mathrm{MHz}$ profile from further discussion.

$\mathrm{OH}$ is the only molecule observed in the PRIMOS data that is more typically present in the most diffuse gas of the CNM and in the WNM than in the higher density regions. It is formed through warm temperature chemistry which can occur efficiently under conditions of shocks and turbulent dissipation (see e.g., Godard et al. 2009, 2014, and references therein).
Turbulent dissipation in particular is believed to significantly impact the chemistry observed in the CNM, with periodic episodes of turbulent dissipation injecting significant amounts of hightemperature molecules into the CNM (Godard et al. 2009). Under the denser, darker, cooler, and less turbulent conditions of the embedded translucent cloud material, $\mathrm{OH}$ should not be formed efficiently, and the reactive radical species should be destroyed efficiently.

As expected from this, the $\mathrm{OH}$ absorption profiles at 1665 and $1667 \mathrm{MHz}$ are more similar to those of small hydride molecules observed with Herschel (e.g., Godard et al. 2012; Indriolo et al. 2015) than to other absorption profiles observed in the PRIMOS data. Comparing the $1667 \mathrm{MHz}$ profile of $\mathrm{OH}$ with $c-\mathrm{C}_{3} \mathrm{H}_{2}$ (Fig. 1), it is apparent that $\mathrm{OH}$ is present in more diffuse material than $c-\mathrm{C}_{3} \mathrm{H}_{2}$. If $c-\mathrm{C}_{3} \mathrm{H}_{2}$ traces $\mathrm{H}_{2}$ even at low extinction (Liszt et al. 2012), this implies that $\mathrm{OH}$ is present in material with very low values of the molecular hydrogen fraction. Furthermore, the $\mathrm{OH}$ absorption profiles indicate that $\mathrm{OH}$ is less abundant in the regions of enhanced density. This is evident as the profiles do not contain significant optical depths within the narrow peaks corresponding to the enhanced $c-\mathrm{C}_{3} \mathrm{H}_{2}$ absorption. These trends are consistent with the theoretical picture of $\mathrm{OH}$ formation.

Finally, we comment on the values of $N_{\mathrm{OH}} / N_{\mathrm{H}_{2}}$ derived. We determine a median value of $N_{\mathrm{OH}} / N_{\mathrm{H}_{2}}=1.1 \times 10^{-6}$. In the lineof-sight to Sgr A, Karlsson et al. (2013) found slightly higher values of 1.8 to $5.8 \times 10^{-6}$ in line-of-sight clouds associated with the same structures that we observe, namely the EMR (at -130 to $-95 \mathrm{~km} \mathrm{~s}^{-1}$ in our data), the $3-\mathrm{kpc}$ arm (at $-40 \mathrm{~km} \mathrm{~s}^{-1}$ toward Sgr B2), and in the $0 \mathrm{~km} \mathrm{~s}^{-1}$ gas. The values reported here and by Karlsson et al. (2013) are significantly larger than those observed in diffuse and translucent gas toward other sightlines however, with values ranging from $5 \times 10^{-8}$ to $2 \times 10^{-7}$ observed toward W51 and W49N (Neufeld et al. 2002; Wiesemeyer et al. 2003). If we consider the abundance with respect to the total hydrogen column by $N_{\mathrm{H}}=N_{\mathrm{HI}}+2 N_{\mathrm{H}_{2}}$, adopting the HI column densities published in Indriolo et al. (2015, Table 3), we obtain typical values of 3 to $6 \times 10^{-7}$.

While it is not appropriate to adopt the values of $N_{\mathrm{OH}} / N_{\mathrm{H}_{2}}$ in Table 4 as true abundances, as $\mathrm{OH}$ primarily occupies a different phase of the ISM than the $c-\mathrm{C}_{3} \mathrm{H}_{2}$ from which $N_{\mathrm{H}_{2}}$ was estimated, we include the values as they likely have physical significance. Lower than average values may indicate that a cloud is more dominated by higher density material as opposed to WNM and CNM material. Notably, lower than average values are observed in the $-106,-40$, and $0 \mathrm{~km} \mathrm{~s}^{-1}$ clouds, where most other molecules are particularly prominent.

\subsection{2. $\mathrm{H}_{2} \mathrm{CO}$}

Excluding $\mathrm{OH}, \mathrm{H}_{2} \mathrm{CO}$ is the most abundant species measured in this work at typical abundances of a few $\times 10^{-7}$. This is significantly higher, by nearly two orders of magnitude, than measurements in high latitude diffuse clouds and cirrus clouds (Liszt et al. 2006; Turner 1993). With respect to other interstellar environments, these abundances are very high, comparable to those observed in the Orion hot core and molecular ridge (Neill et al. 2014), and two and four orders of magnitude, respectively, larger than what is observed in the dark clouds TMC-1 and in the Horsehead PDR (Ohishi \& Kaifu 1998; Guzmán et al. 2014). In the warm kinetic temperatures and low densities of the diffuse clouds, $\mathrm{H}_{2} \mathrm{CO}$ production is likely dominated by the neutral-neutral reaction of

$\mathrm{O}+\mathrm{CH}_{3} \rightarrow \mathrm{H}_{2} \mathrm{CO}+\mathrm{H}$, 
which proceeds efficiently at warm kinetic temperatures of $T \sim$ $100 \mathrm{~K}$ (Baulch et al. 1992). However, under low extinction conditions, $\mathrm{H}_{2} \mathrm{CO}$ is not predicted to be nearly as abundant as observed due to photodissociation (Liszt et al. 2006).

The abundance of $\mathrm{H}_{2} \mathrm{CO}$ varies by a factor of $\sim 4$ within the observed clouds and does not exhibit an apparent correlation with $N_{c-\mathrm{C}_{3} \mathrm{H}_{2}}$; assuming that the abundance of $c-\mathrm{C}_{3} \mathrm{H}_{2}$ is constant with respect to $\mathrm{H}_{2}$, the abundance of $\mathrm{H}_{2} \mathrm{CO}$ therefore does not correlate with $\mathrm{N}_{\mathrm{H}_{2}}$. However, the $\mathrm{H}_{2} \mathrm{CO}$ abundance does exhibit a positive linear correlation with the observed abundance of CS (Fig. 10). Liszt et al. (2006) also demonstrated that the abundances of $\mathrm{H}_{2} \mathrm{CO}$ and $\mathrm{CS}$ are tightly correlated, whereas $\mathrm{H}_{2} \mathrm{CO}$ is not correlated with hydrocarbons like $c-\mathrm{C}_{3} \mathrm{H}_{2}$. Once again, the abundance ratios that we observe in the line-of-sight clouds to Sgr B2 are different than what has been reported in other sightlines; whereas $N_{\mathrm{H}_{2} \mathrm{CO}} / N_{\mathrm{CS}}$ was measured to be approximately 2 to 3 by Liszt et al. (2006), we obtain higher values of $\sim 20$ to 30 . Inspecting the profiles of $\mathrm{H}_{2} \mathrm{CO}$ and $\mathrm{CS}$, it is unclear why the two species should correlate well. Whereas CS appears to prefer higher density regions of the diffuse clouds, the absorption profile of $\mathrm{H}_{2} \mathrm{CO}$ includes moderately broad absorption components indicating that $\mathrm{H}_{2} \mathrm{CO}$ is likely abundant in a larger fraction of the cloud volume. If a warm temperature gas phase reaction with neutral oxygen dominates the formation of $\mathrm{H}_{2} \mathrm{CO}$ (Eq. (11)), then it is reasonable that $\mathrm{H}_{2} \mathrm{CO}$ is fairly widespread. It is curious however that the abundances of $\mathrm{H}_{2} \mathrm{CO}$ and $\mathrm{CS}$ are correlated when they appear to occupy distinct pockets of gas.

\subsection{3. $\mathrm{SiO}$}

Abundances of $\mathrm{SiO}$ in the line-of-sight to Sgr B2(M) have been measured by Greaves \& Nyman (1996), and abundance patterns in the sightlines to Sgr B2(M) and $\mathrm{W} 49 \mathrm{~N}$ were discussed by Greaves et al. (1996). In the line-of-sight toward Sgr B2(M), values measured by Greaves \& Nyman (1996) varied by an order of magnitude, from $3 \times 10^{-10}$ in the $-40 \mathrm{~km} \mathrm{~s}^{-1}$ cloud, to 2 and $3 \times 10^{-9}$ in the -106 and $-75 \mathrm{~km} \mathrm{~s}^{-1}$ clouds, respectively. In our data, we observe values in a similar range, of $1.5 \times 10^{-10}$ to $1.7 \times 10^{-9}$. We observe lower values in clouds located in the Galactic disk, namely $\sim 4 \times 10^{-10}$ in the -40 and $-23 \mathrm{~km} \mathrm{~s}^{-1}$ clouds, and higher values of 1.3 to $1.7 \times 10^{-9}$ in the $-73,0$, and $+25 \mathrm{~km} \mathrm{~s}^{-1}$ clouds located in the Galactic bar and center. We observe a very low value in the $-58 \mathrm{~km} \mathrm{~s}^{-1}$ cloud, which is surprising because this is believed to be located internal to the Galactic bar or center.

The profile of $\mathrm{SiO}$ may also hold clues to the nature of the gas in which $\mathrm{SiO}$ is most abundant. The absorbing components at $-73,-40$, and $-23 \mathrm{~km} \mathrm{~s}^{-1}$ in particular appear to be broader and more smoothly varying than in profiles of most other molecules. The profile does not contain the narrow absorption features present in the profiles of most other species observed in this study, and particularly of CS and SO. Therefore, it does not appear that $\mathrm{SiO}$ is enhanced in the densest material. The components of $\mathrm{SiO}$ may be shock broadened and $\mathrm{SiO}$ may be abundant only in recently shocked material. In this scenario, $\mathrm{SiO}$ could be confined to a small spatial region on a shock front, or could be spatially extended, perhaps occupying gas with more recent episodes of turbulence, which is believed to be important in the diffuse ISM (Godard et al. 2009). Alternatively, the SiO abundance could be governed more directly by the X-ray or CR flux in the medium, and the species may inhabit more diffuse conditions than most molecules observed in this study.

A deeper investigation of the formation and destruction of $\mathrm{SiO}$ in diffuse clouds could help elucidate the structure of the diffuse clouds. For instance, if the $\mathrm{SiO}$ abundance is most directly governed by shocks in these environments, then this data may indicate that the high density clumps in which CS and SO are abundant are not subjected to the shocks associated with turbulent dissipation, and tend to be undisrupted within a turbulent medium. This would put a limit on the size scales of the clumps and advance our understanding of the physical processes ongoing in this material.

\subsection{Linear hydrocarbons: $l-C_{3} H$ and $l-C_{3} H^{+}$}

The linear hydrocarbon species $l-\mathrm{C}_{3} \mathrm{H}$ has been a known interstellar molecule for more than three decades, and is present in multiple distinct environments including evolved stars (Thaddeus et al. 1985; Pardo \& Cernicharo 2007), dark clouds (Thaddeus et al. 1985), and PDRs (Pety et al. 2012). The cation of this species, $l-\mathrm{C}_{3} \mathrm{H}^{+}$, was first detected much more recently, by Pety et al. (2012) in the Horsehead PDR. Whereas most molecules observed in the PDR, including $l-\mathrm{C}_{3} \mathrm{H}$ (Pety et al. 2012), are also detected in a dense, UV-shielded core in the Horsehead nebula (e.g., Guzmán et al. 2014), $l-\mathrm{C}_{3} \mathrm{H}^{+}$was not observed toward the core, providing the first indication that it is abundant only in the presence of far-UV radiation. Since the initial detection, $l-\mathrm{C}_{3} \mathrm{H}^{+}$has also been detected in the Orion Bar PDR (McGuire et al. 2014b) and in material in Sgr B2 (McGuire et al. 2013) that likely contains a high UV and Xray flux (Corby et al. 2015; Goicoechea et al. 2003). Additionally, $l-\mathrm{C}_{3} \mathrm{H}^{+}$has been rigorously detected in three other PDR sources, and it is tentatively detected in two additional PDRs (B. McGuire, priv. comm.). Despite a search for $l-\mathrm{C}_{3} \mathrm{H}^{+}$toward 35 additional sources including hot cores, hot corinos, and evolved stars, the species was not detected (McGuire et al. $2014 \mathrm{a}, \mathrm{b})$. Thus, $l-\mathrm{C}_{3} \mathrm{H}^{+}$appears to be an excellent indicator of a UV-enhanced environment.

Although the profile of $l-\mathrm{C}_{3} \mathrm{H}$ is difficult to interpret due to the presence of hyperfine structure, line confusion, and weak signal, we determine reasonably consistent $l-\mathrm{C}_{3} \mathrm{H}$ abundances of 1.5 to $5 \times 10^{-10}$. These values are slightly higher than the observed values in the Horsehead PDR of $(1.4 \pm$ $0.7) \times 10^{-10}$. Adopting the $l-\mathrm{C}_{3} \mathrm{H}$ column derived in Sgr B2(N) (McGuire et al. 2013) and the molecular hydrogen column derived in Appendix A of Quan et al. (2016), a similar value of 1 to $2 \times 10^{-10}$ is present in the PDR/XDR material in Sgr B2(N).

Measured $l-\mathrm{C}_{3} \mathrm{H}^{+}$abundances in this work range from 4 to $11 \times 10^{-11}$, although they have considerable uncertainties of about 50 percent given the weak signal and the presence of line blending. These values are slightly higher than in the Horsehead PDR $\left(3 \times 10^{-11}\right)$ and in Sgr B2 $\left(2\right.$ to $\left.3 \times 10^{-11}\right)$. For clouds with measured abundances of both $l-\mathrm{C}_{3} \mathrm{H}$ and $l-\mathrm{C}_{3} \mathrm{H}^{+}$in this survey, the ratio of $l-\mathrm{C}_{3} \mathrm{H} / l-\mathrm{C}_{3} \mathrm{H}^{+}$ranges from $\sim 2.5$ to 5.5 . The ratios are therefore quite similar to the value of $\sim 4$ observed in the Horsehead PDR, and comparable to the value of $\sim 6$ observed in the PDR/XDR gas in Sgr B2. We also note that the absorbing components of $l-\mathrm{C}_{3} \mathrm{H}^{+}$are broader than observed in the profiles of most other species in this study. While the weak signal may contribute to this, it may be that $l-\mathrm{C}_{3} \mathrm{H}^{+}$is more spatially extended than most species observed here, preferentially populating the more diffuse material rather than the embedded clumps. This would be expected if $l-\mathrm{C}_{3} \mathrm{H}^{+}$is formed only in the presence of a strong far-UV field.

PDR models do a poor job of accounting for the abundances of most molecules observed in the diffuse ISM (e.g., Godard et al. 2009, 2014) despite the high UV fields in these environments. Yet it will be interesting to determine whether 
PDR models can account for the observed abundances of these two linear hydrocarbon species in the environments studied here. Pety et al. (2012) was able to reproduce the observed abundances of both species in the Horsehead PDR using a PDR model with conditions appropriate in that source. While the mean densities are much lower in the diffuse and translucent clouds considered here, it is plausible that the observed abundances of $l-\mathrm{C}_{3} \mathrm{H}$ and $l-\mathrm{C}_{3} \mathrm{H}^{+}$may also be consistent with PDR model predictions. A more recent study of the molecules suggests that they are formed from the photo-destruction of PAHs, with $l-\mathrm{C}_{3} \mathrm{H}^{+}$being an intermediate to the formation of $l-\mathrm{C}_{3} \mathrm{H}$ (Guzmán et al. 2015). Due to the widespread presence of the Diffuse Interstellar Bands (DIBs) in diffuse cloud spectra (Low et al. 1984; Tielens 2008), it is generally accepted that PAHs are prevalent in the diffuse ISM, although there are some challenges to this model (e.g., Salama \& Ehrenfreund 2014). Assuming that PAHs are common in the diffuse ISM, if $l-\mathrm{C}_{3} \mathrm{H}$ and $l-\mathrm{C}_{3} \mathrm{H}^{+}$form from the destruction of PAHs, then their abundances should not be enhanced in the higher density clumps, and should be sensitive to PAH abundances and the UV field within the cloud. The moderately broad absorption components that characterize the $l-\mathrm{C}_{3} \mathrm{H}$ and $l-\mathrm{C}_{3} \mathrm{H}^{+}$profiles are consistent with these expectations. Further observational study of $l-\mathrm{C}_{3} \mathrm{H}^{+}$and PAHs, including follow-up on sightlines with strong DIB features to determine whether $l-\mathrm{C}_{3} \mathrm{H}^{+}$is present, could further test this model.

\section{Conclusions}

We have compiled the line profiles of 26 molecular lines from 11 molecules with absorption by diffuse and translucent clouds in the line-of-sight to Sgr B2(N) with the GBT PRIMOS data. The data reveal the presence of $\sim 10$ kinematically separate clouds. In each cloud, we determined the column densities of observed molecules and estimated the molecular hydrogen column density by assuming a constant abundance of $c-\mathrm{C}_{3} \mathrm{H}_{2}$ with respect to $\mathrm{H}_{2}$. Conversion from the $c-\mathrm{C}_{3} \mathrm{H}_{2}$ column density provides a new method for measuring the molecular hydrogen column $\left(N_{\mathrm{H}_{2}}\right)$, which is, for numerous reasons described in Sect. 4.1, preferable to conversion from isotopologues of more abundant molecules like $\mathrm{CO}$ and $\mathrm{HCO}^{+}$. We then converted the molecular column densities to abundances and considered the abundance patterns. We summarize new results below, including systematic trends with Galactocentric distance.

By comparing the $\mathrm{H}_{2}$ column densities measured by conversion from $N_{c-\mathrm{C}_{3} \mathrm{H}_{2}}$ to previous measurements of $\mathrm{HI}$ absorption (Indriolo et al. 2015), we note a stark decrease in the molecular hydrogen fraction with Galactocentric distance. In the Galactic disk, we estimate a molecular fraction of 0.65 , slightly higher than typical values of 0.4 measured in diffuse clouds that contain heavy molecules (Liszt et al. 2012); in material near the $3 \mathrm{kpc}$ arm, the molecular fraction is significantly greater, at $\sim 0.85$, and in the Galactic center material, the data indicate that $\sim 95$ percent of the hydrogen is in molecular form.

We see additional trends with Galactocentric distance in the isotope ratios of ${ }^{12} \mathrm{C} /{ }^{13} \mathrm{C},{ }^{32} \mathrm{~S} /{ }^{34} \mathrm{~S}$, and ${ }^{28} \mathrm{Si} /{ }^{29} \mathrm{Si}$. Whereas the ratios of $\mathrm{H}_{2} \mathrm{CO} / \mathrm{H}_{2}^{13} \mathrm{CO}$ are higher than previous estimates of ${ }^{12} \mathrm{C} /{ }^{13} \mathrm{C}$ indicating the presence of isotope fractionation, $c-\mathrm{C}_{3} \mathrm{H}_{2} / c-\mathrm{H}^{13} \mathrm{CCCH}$ column density ratios show a trend of increasing value with Galactocentric distance, consistent with agreed upon values in the Galaxy. ${ }^{28} \mathrm{Si} /{ }^{29} \mathrm{Si}$ ratios also exhibit a gradient with Galactocentric distance, from a value of 13 in the Galactic center to $\sim 21$ in the Galactic bar. On the other hand, ${ }^{32} \mathrm{~S} /{ }^{34} \mathrm{~S}$, measured by $\mathrm{CS} / \mathrm{C}^{34} \mathrm{~S}$, does not show any apparent trend with Galactocentric distance. Furthermore, values measured in this work are lower than previous measurements by a factor of $\sim 3$ to 4 , with typical $\mathrm{CS} / \mathrm{C}^{34} \mathrm{~S}$ of 5 to 10 .

The abundances of $\mathrm{OH}$ are significantly higher, by 1-2 orders of magnitude, in clouds along this sightline compared to diffuse clouds at high Galactic longitude. The line profiles indicate that $\mathrm{OH}$ preferentially resides in low density gas with low molecular hydrogen fractions, in a distinct environment from the other species presented in this study. In clouds with more pronounced absorption by the other molecules featured in this paper, the values of $N_{\mathrm{OH}} / N_{\mathrm{H}_{2}}$ are lower than average, so that $N_{\mathrm{OH}} / N_{\mathrm{H}_{2}}$ may indicate the degree to which a cloud is dominated by very diffuse or more moderate density gas. These results are expected from our current understanding of $\mathrm{OH}$ formation and destruction in the diffuse ISM.

Additionally, we observe linear hydrocarbon species $l-\mathrm{C}_{3} \mathrm{H}$ and $l-\mathrm{C}_{3} \mathrm{H}^{+}$in similar abundance ratios to observations in the Horsehead PDR and in PDR/XDR material in Sgr B2(N). $l$ $\mathrm{C}_{3} \mathrm{H}^{+}$is an interesting species in that it may uniquely trace $\mathrm{UV}$-enhancement, and follow-up studies on the UV-induced formation of $l-\mathrm{C}_{3} \mathrm{H}^{+}$and $l-\mathrm{C}_{3} \mathrm{H}$ in diffuse clouds could prove interesting.

The abundance trends and line profiles of CS indicate that the species may provide a good diagnostic of the physical conditions and structure of diffuse and translucent clouds, and the observations indicate systematic differences in the physical structure of clouds located internal to the Galactic bar compared to the disk. Whereas $c-\mathrm{C}_{3} \mathrm{H}_{2}$ has been shown to have a constant abundance in diffuse and translucent clouds (Liszt et al. 2012), the abundance of CS varies quite significantly in different clouds. No systematic offset is apparent in the abundances within clouds located in the Galactic center and bar compared to the disk, and we do not observe a trend with $N_{\mathrm{H}_{2}}$. However, the CS line profile includes multiple strong, narrow $(\Delta v \lesssim 1)$ absorption features in clouds located in the Galactic disk, suggesting the presence of multiple embedded sources with elevated CS abundances and presumably higher densities. Clouds in the Galactic center and bar instead contain CS ubiquitously over the same moderately broad velocity ranges as $c-\mathrm{C}_{3} \mathrm{H}_{2}$ and $\mathrm{H}_{2} \mathrm{CO}$.

Abundances of $\mathrm{H}_{2} \mathrm{CO}$ and $\mathrm{SO}$ are positively correlated with the $\mathrm{CS}$ abundance, confirming the correlation between $\mathrm{H}_{2} \mathrm{CO}$ and CS observed by Liszt et al. (2006). Additionally CSbearing molecules including $\mathrm{HCS}^{+}, \mathrm{H}_{2} \mathrm{CS}$, and $\mathrm{CCS}$ are many orders of magnitude overabundant compared to model predictions. These CS-bearing molecules may prove excellent diagnostics for the cloud physical conditions, however an improved theoretical treatment of sulfur-bearing chemistry is first required.

Finally, the abundance of $\mathrm{SiO}$ varies significantly in the clouds, and the broad profiles of $\mathrm{SiO}$ may suggest that it is more spatially extended than most of the molecules and does not reside in higher density embedded clumps. If $\mathrm{SiO}$ indicates shocks in these environments, then it is apparent that the $-106,-73$, and $\sim 0 \mathrm{~km} \mathrm{~s}^{-1}$ material is highly shocked, with the highest $\mathrm{SiO}$ abundance in the $-73 \mathrm{~km} \mathrm{~s}^{-1}$ cloud, and that the $-40 \mathrm{~km} \mathrm{~s}^{-1}$ cloud in the Galactic disk has recently experienced shocks. Further, if $\mathrm{SiO}$ inhabits recently shocked material, then the broad line profiles of $\mathrm{SiO}$ compared to the narrow, jagged profiles of $\mathrm{CS}$ and SO, suggest that the dense clumps containing enhanced abundances of CS and SO are not disrupted by shocks, putting constraints on their size scales.

Acknowledgements. J.F.C. gratefully acknowledges partial support from funds provided by the University of Virginia. B.A.M. is a Jansky Fellow of the National Radio Astronomy Observatory. E.H. wishes to thank the National Science Foundation (US) for continued support of his research program in astrochemistry. Support for this work was provided by the NSF through the Grote Reber 
Fellowship Program administered by Associated Universities, Inc./National Radio Astronomy Observatory. The National Radio Astronomy Observatory is a facility of the National Science Foundation operated under cooperative agreement by Associated Universities, Inc.

\section{References}

Amo-Baladrón, M. A., Martín-Pintado, J., Morris, M. R., Muno, M. P., \& Rodríguez-Fernández, N. J. 2009, ApJ, 694, 943

Baulch, D. L., Cobos, C. J., Cox, R. A., et al. 1992, J. Phys. Chem. Ref. Data, 21, 411

Chin, Y.-N., Henkel, C., Whiteoak, J. B., Langer, N., \& Churchwell, E. B. 1996, A\&A, 305, 960

Corby, J. F., Jones, P. A., Cunningham, M. R., et al. 2015, MNRAS, 452, 3969

Crockett, N. R., Bergin, E. A., Neill, J. L., et al. 2014, ApJ, 787, 112

Douglas, A. E., \& Herzberg, G. 1941, ApJ, 94, 381

Drdla, K., Knapp, G. R., \& van Dishoeck, E. F. 1989, ApJ, 345, 815

Ebisawa, Y., Inokuma, H., Sakai, N., et al. 2015, ApJ, 815, 13

Elitzur, M. 1992, ARA\&A, 30, 75

Frerking, M. A., Wilson, R. W., Linke, R. A., \& Wannier, P. G. 1980, ApJ, 240, 65

Gerin, M., Falgarone, E., Joulain, K., et al. 1997, A\&A, 318, 579

Gerin, M., de Luca, M., Black, J., et al. 2010a, A\&A, 518, L110

Gerin, M., de Luca, M., Goicoechea, J. R., et al. 2010b, A\&A, 521, L16

Gerin, M., Kaźmierczak, M., Jastrzebska, M., et al. 2011, A\&A, 525, A116

Godard, B., Falgarone, E., \& Pineau Des Forêts, G. 2009, A\&A, 495, 847

Godard, B., Falgarone, E., Gerin, M., et al. 2012, A\&A, 540, A87

Godard, B., Falgarone, E., \& Pineau des Forêts, G. 2014, A\&A, 570, A27

Goicoechea, J. R., Rodríguez-Fernández, N. J., \& Cernicharo, J. 2003, Astron. Nachr. Suppl., 324, 139

Goicoechea, J. R., Pety, J., Gerin, M., et al. 2006, A\&A, 456, 565

Gray, M. D., Doel, R. C., \& Field, D. 1991, MNRAS, 252, 30

Greaves, J. S., \& Nyman, L.-A. 1996, A\&A, 305, 950

Greaves, J. S., \& Williams, P. G. 1994, A\&A, 290, 259

Greaves, J. S., White, G. J., Ohishi, M., Hasegawa, T., \& Sunada, K. 1992, A\&A, 260,381

Greaves, J. S., Ohishi, M., \& Nyman, L.-A. 1996, A\&A, 307, 898

Güver, T., \& Özel, F. 2009, MNRAS, 400, 2050

Guzmán, V. V., Pety, J., Gratier, P., et al. 2014, Faraday Discussions, 168, 103

Guzmán, V. V., Pety, J., Goicoechea, J. R., et al. 2015, ApJ, 800, L33

Huettemeister, S., Wilson, T. L., Mauersberger, R., et al. 1995, A\&A, 294, 667

Huettemeister, S., Dahmen, G., Mauersberger, R., et al. 1998, A\&A, 334, 646 Indriolo, N., Neufeld, D. A., Gerin, M., et al. 2015, ApJ, 800, 40

Irvine, W. M., Goldsmith, P. F., \& Hjalmarson, A. 1987, in Interstellar Processes, eds. D. J. Hollenbach, \& H. A. Thronson, Jr., Astrophys. Space Sci. Lib., 134, 561

Jones, P. A., Burton, M. G., Cunningham, M. R., et al. 2012, MNRAS, 419, 2961

Karlsson, R., Sandqvist, ̊., Hjalmarson, ̊., et al. 2013, A\&A, 554, A141

Langer, W. D., Graedel, T. E., Frerking, M. A., \& Armentrout, P. B. 1984, ApJ 277, 581

Le Petit, F., Ruaud, M., Bron, E., et al. 2016, A\&A, 585, A105

Linke, R. A., Stark, A. A., \& Frerking, M. A. 1981, ApJ, 243, 147

Liszt, H., Sonnentrucker, P., Cordiner, M., \& Gerin, M. 2012, ApJ, 753, L28

Liszt, H. S., Lucas, R., \& Pety, J. 2006, A\&A, 448, 253

Low, F. J., Young, E., Beintema, D. A., et al. 1984, ApJ, 278, L19

Lucas, R., \& Liszt, H. S. 1993, A\&A, 276, L33
Lucas, R., \& Liszt, H. S. 2000, A\&A, 358, 1069

Lucas, R., \& Liszt, H. S. 2002, A\&A, 384, 1054

Mauersberger, R., Henkel, C., Langer, N., \& Chin, Y.-N. 1996, A\&A, 313, L1

McDowell, R. S. 1988, J. Chem. Phys., 88, 356

McDowell, R. S. 1990, J. Chem. Phy., 93, 2801

McGuire, B. A., Carroll, P. B., Loomis, R. A., et al. 2013, ApJ, 774, 56

McGuire, B. A., Carroll, P. B., Gratier, P., et al. 2014a, ApJ, 783, 36

McGuire, B. A., Carroll, P. B., Sanders, J. L., et al. 2014b, MNRAS, 442, 2901

McKellar, A. 1940, PASP, 52, 187

Milam, S. N., Savage, C., Brewster, M. A., Ziurys, L. M., \& Wyckoff, S. 2005, ApJ, 634, 1126

Montaigne, H., Geppert, W. D., Semaniak, J., et al. 2005, ApJ, 631, 653

Morris, M., \& Serabyn, E. 1996, ARA\&A, 34, 645

Müller, H. S. P., Schlöder, F., Stutzki, J., \& Winnewisser, G. 2005, J. Mol. Struct., 742,215

Neill, J. L., Bergin, E. A., Lis, D. C., et al. 2014, ApJ, 789, 8

Neufeld, D. A., Kaufman, M. J., Goldsmith, P. F., Hollenbach, D. J., \& Plume, R. 2002, ApJ, 580, 278

Neufeld, D. A., Godard, B., Gerin, M., et al. 2015, A\&A, 577, A49

Nyman, L.-A. 1984, A\&A, 141, 323

Ohishi, M., \& Kaifu, N. 1998, Faraday Discussions, 109, 205

Ohishi, M., Irvine, W. M., \& Kaifu, N. 1992, in Astrochemistry of Cosmic Phenomena, ed. P. D. Singh, IAU Symp., 150, 171

Oka, T., Geballe, T. R., Goto, M., Usuda, T., \& McCall, B. J. 2005, ApJ, 632, 882

Pardo, J. R., \& Cernicharo, J. 2007, ApJ, 654, 978

Penzias, A. A. 1981, ApJ, 249, 513

Pety, J., Teyssier, D., Fossé, D., et al. 2005, A\&A, 435, 885

Pety, J., Gratier, P., Guzmán, V., et al. 2012, A\&A, 548, A68

Pickett, H. M., Poynter, R. L., Cohen, E. A., et al. 1998, J. Quant. Spectr. Rad. Transf., 60, 883

Qin, S.-L., Schilke, P., Comito, C., et al. 2010, A\&A, 521, L14

Quan, D., Herbst, E., Corby, J. F., Durr, A., \& Hassel, G. 2016, ApJ

Remijan, A. J., Markwick-Kemper, A., \& ALMA Working Group on Spectral Line Frequencies. 2007, in AAS Meeting Abstracts, BAAS, 39, 963

Royster, M. J., \& Yusef-Zadeh, F. 2014, in The Galactic Center: Feeding and Feedback in a Normal Galactic Nucleus, eds. L. O. Sjouwerman, C. C. Lang, \& J. Ott, IAU Symp., 303, 92

Salama, F., \& Ehrenfreund, P. 2014, in The Diffuse Interstellar Bands, eds. J. Cami, \& N. L. J. Cox, IAU Symp., 297, 364

Sheffer, Y., Rogers, M., Federman, S. R., et al. 2008, ApJ, 687, 1075

Snow, T. P., \& McCall, B. J. 2006, ARA\&A, 44, 367

Sonnentrucker, P., Welty, D. E., Thorburn, J. A., \& York, D. G. 2007, ApJS, 168, 58

Stahl, O., Casassus, S., \& Wilson, T. 2008, A\&A, 477, 865

Swings, P., \& Rosenfeld, L. 1937, ApJ, 86, 483

Thaddeus, P., Gottlieb, C. A., Hjalmarson, A., et al. 1985, ApJ, 294, L49

Tielens, A. G. G. M. 2008, ARA\&A, 46, 289

Turner, B. E. 1993, ApJ, 410, 140

Vastel, C., Polehampton, E. T., Baluteau, J.-P., et al. 2002, ApJ, 581, 315

Whiteoak, J. B., \& Gardner, F. F. 1979, MNRAS, 188, 445

Wiesemeyer, H., Thum, C., Morris, D., \& Sievers, A. W. 2003, Astron. Nachr. Suppl., 324, 24

Wiesemeyer, H., Güsten, R., Heyminck, S., et al. 2016, A\&A, 585, A76

Wilson, T. L. 1999, Rep. Prog. Phys., 62, 143

Wilson, T. L., \& Rood, R. 1994, ARA\&A, 32, 191

Winkel, B., Kerp, J., Flöer, L., et al. 2016, A\&A, 585, A41

Wirström, E. S., Bergman, P., Black, J. H., et al. 2010, A\&A, 522, A19

Wolfire, M. G., Hollenbach, D., \& McKee, C. F. 2010, ApJ, 716, 1191 


\section{Appendix A: Spectral line profiles and computed column densities}

In this appendix we present the line profiles of all molecular lines listed in Table 1. These profiles constitute all transitions with detected line-of-sight absorption by the 11 molecules and isotopologues analyzed in this study. We additionally provide the best fit molecular column densities derived with an assumed excitation temperature of $T_{\mathrm{ex}}=3 \mathrm{~K}$, and the errors provided are determined as described in Sect. 3.3.

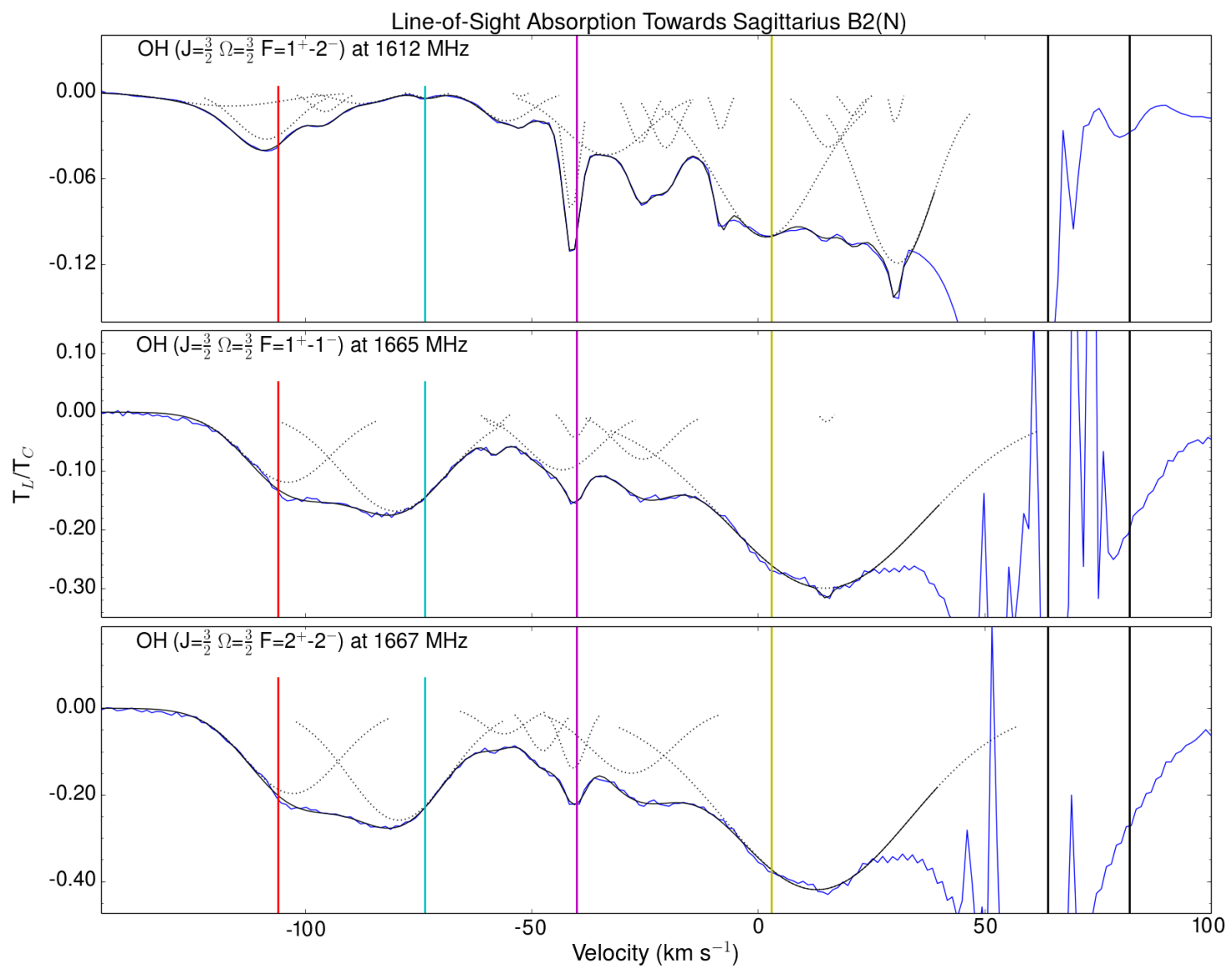

Fig. A.1. Absorption profiles of $\mathrm{OH}$ are shown in blue. Black vertical lines indicate the velocities of line absorption by Sgr B2 at +64 and $+82 \mathrm{~km} \mathrm{~s}^{-1}$, and colored lines are located at $-106,-73.5,-40$, and $+3 \mathrm{~km} \mathrm{~s}^{-1}$. Hyperfine or A/E structure for each of these velocity components is indicated by dotted vertical lines of the same color. The data are overlaid by individual Gaussian components fit (black dotted lines), and by the total fit to the profile (black solid line). 
J. F. Corby et al.: The molecular chemistry of diffuse and translucent clouds in the line-of-sight to Sgr B2

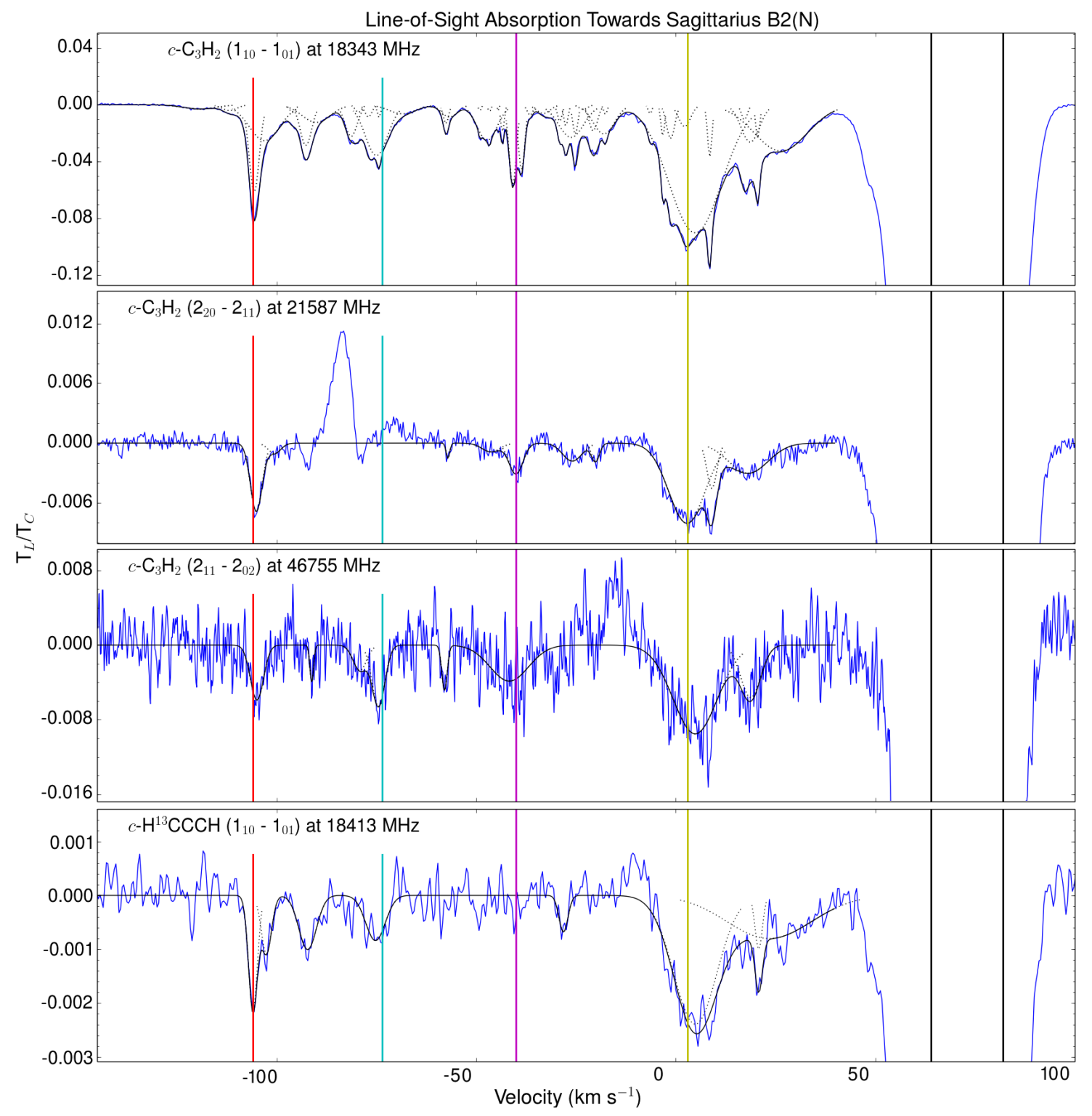

Fig. A.2. Absorption profiles of $c-\mathrm{C}_{3} \mathrm{H}_{2}$ and $c-\mathrm{H}^{13} \mathrm{CCCH}$ are shown in blue. Black vertical lines indicate the velocities of line absorption by Sgr B2 at +64 and $+82 \mathrm{~km} \mathrm{~s}^{-1}$, and colored lines are located at $-106,-73.5,-40$, and $+3 \mathrm{~km} \mathrm{~s}^{-1}$. The data are overlaid by individual Gaussian components fit (black dotted lines), and by the total fit to the profile (black solid line). 


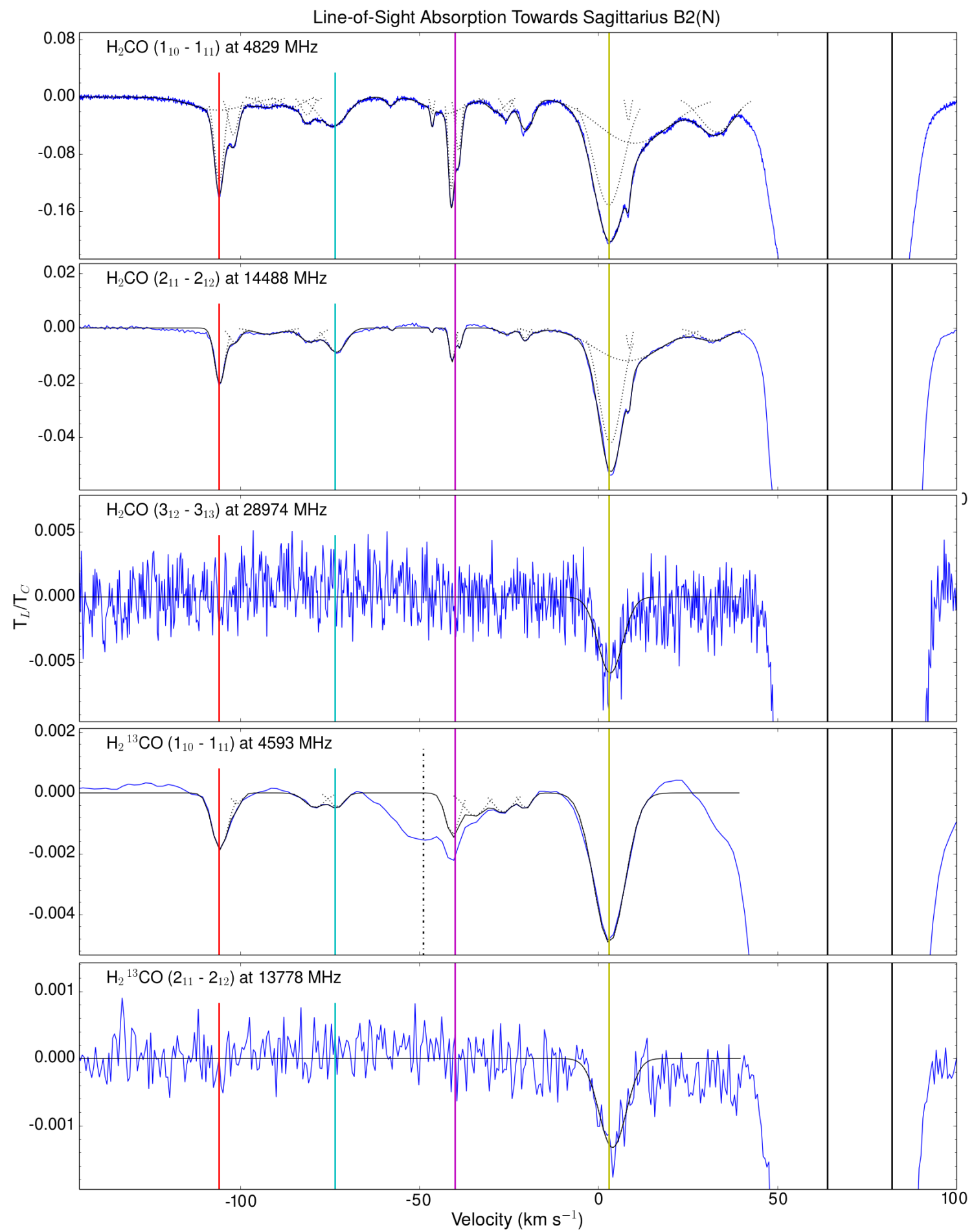

Fig. A.3. Absorption profiles of $\mathrm{H}_{2} \mathrm{CO}$ and $\mathrm{H}_{2}^{13} \mathrm{CO}$ are shown in blue. Black vertical lines indicate the velocities of line absorption by Sgr $\mathrm{B} 2$ at +64 and $+82 \mathrm{~km} \mathrm{~s}^{-1}$, and colored lines are located at $-106,-73.5,-40$, and $+3 \mathrm{~km} \mathrm{~s}^{-1}$. The data are overlaid by individual Gaussian components fit (black dotted lines), and by the total fit to the profile (black solid line). In the profile of $\mathrm{H}_{2}^{13} \mathrm{CO}$ at $4593 \mathrm{MHz}$, the black dashed-dotted line marks an unidentified transition, and the black line trace of the total fit includes only absorption attributed to $\mathrm{H}_{2}^{13} \mathrm{CO}$. 
J. F. Corby et al.: The molecular chemistry of diffuse and translucent clouds in the line-of-sight to Sgr B2

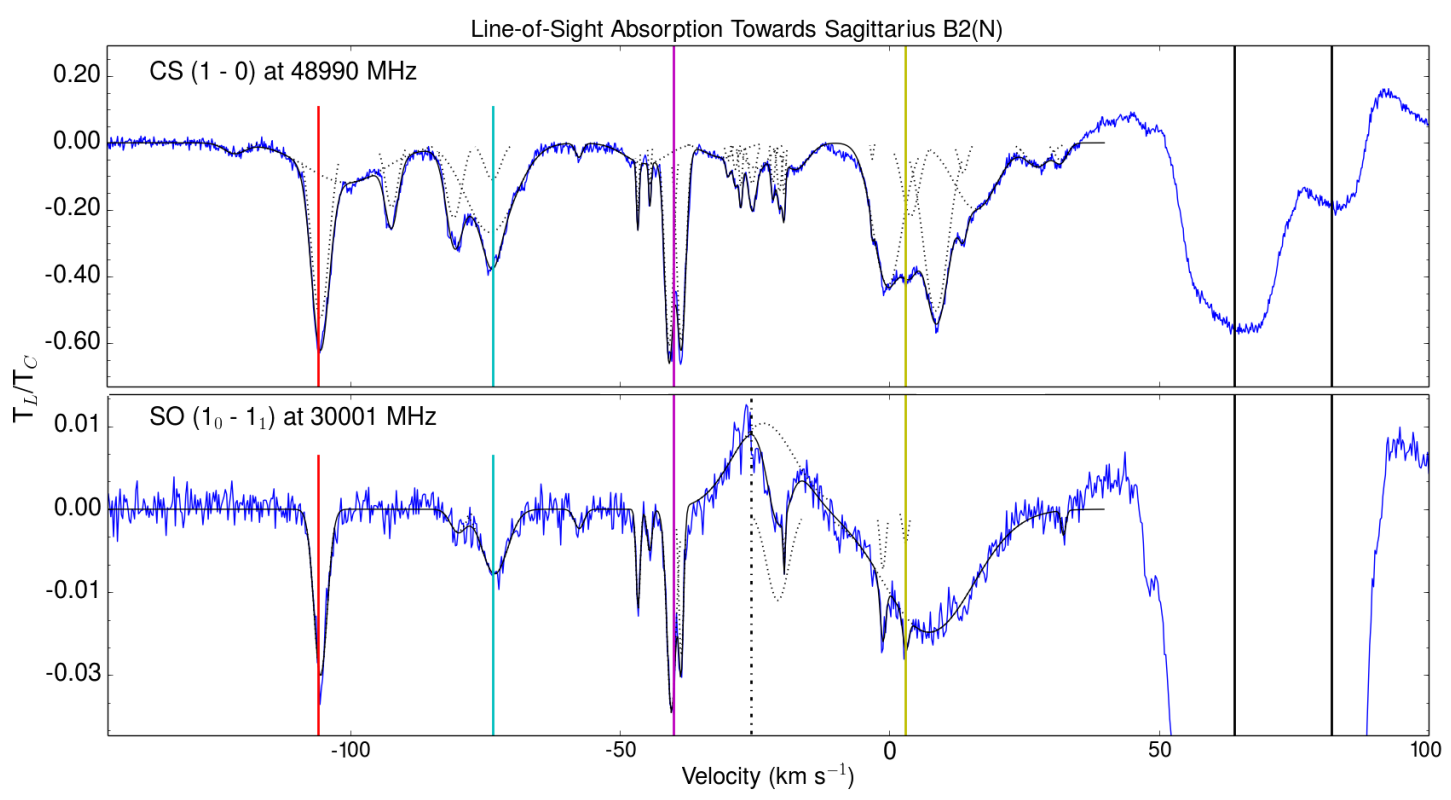

Fig. A.4. Absorption profiles of SO and CS are shown in blue. Black vertical lines indicate the velocities of line absorption by Sgr B2 at +64 and $+82 \mathrm{~km} \mathrm{~s}^{-1}$, and colored lines are located at $-106,-73.5,-40$, and $+3 \mathrm{~km} \mathrm{~s}^{-1}$. The data are overlaid by individual Gaussian components fit (black dotted lines), and by the total fit to the profile (black solid line).

Table A.1. Derived molecular column densities.

\begin{tabular}{|c|c|c|c|c|c|c|c|c|c|c|c|c|}
\hline \multirow[t]{2}{*}{ Species } & \multicolumn{11}{|c|}{ Absorption cloud velocity $\left(\mathrm{km} \mathrm{s}^{-1}\right)$} & \multirow{2}{*}{$\begin{array}{l}\text { Units } \\
\left(\mathrm{cm}^{-2}\right)\end{array}$} \\
\hline & -120 & -106 & -92 & -80 & -73 & -58 & -47 & -40 & -23 & 0 & +20 & \\
\hline $\mathrm{OH}$ & $0.31(2)$ & $2.2(1)$ & $2.2(1)$ & $1.79(6)$ & $2.3(1)$ & $0.66(6)$ & $1.1(1)$ & $1.2(1)$ & $3.8(3)$ & $8.4(7)$ & $3.4(4)$ & $10^{15}$ \\
\hline$c-\mathrm{C}_{3} \mathrm{H}_{2}$ & $0.43(4)$ & $8.0(6)$ & $4.3(2)$ & $2.8(1)$ & $5.8(3)$ & $1.29(8)$ & $3.5(3)$ & $4.5(4)$ & $8.8(9)$ & $30(3)$ & $7.9(13)$ & $10^{12}$ \\
\hline \multicolumn{2}{|c|}{$c-\mathrm{H}^{13} \mathrm{CCCH}$} & $3.1(7)$ & $1.8(6)$ & & $1.4(7)$ & & & $<0.5$ & $0.6(3)$ & $11(2)$ & $3.2(6)$ & $10^{11}$ \\
\hline $\mathrm{H}_{2} \mathrm{CO}$ & $0.69(6)$ & $15(3)$ & $<3.1$ & $4.6(5)$ & $7.9(3)$ & $1.0(1)$ & $4.0(2)$ & $12.5(6)$ & $10.4(6)$ & $71(20)$ & $8.1(8)$ & $10^{14}$ \\
\hline $\mathrm{H}_{2}^{13} \mathrm{CO}$ & & $2.7(2)$ & & $0.5(1)$ & $0.8(3)$ & & & $1.8(8)$ & $2.2(3)$ & $12.6(9)$ & & $10^{13}$ \\
\hline SO & & $3.9(3)$ & $<0.4$ & $0.6(1)$ & $2.9(3)$ & $0.3(1)$ & $1.1(2)$ & $4.5(3)$ & $4.0(11)$ & 17(1) & $3.0(2)$ & $10^{12}$ \\
\hline $\mathrm{CS}$ & $0.22(2)$ & $5.2(3)^{*}$ & $1.54(8)$ & $1.9(1)$ & $3.9(2)$ & $0.11(2)$ & $0.82(5)$ & $4.4(2)^{*}$ & $2.5(1)$ & $11.3(6)^{*}$ & $1.48(7)$ & $10^{13}$ \\
\hline $\mathrm{C}^{34} \mathrm{~S}$ & & $6.0(4)$ & $0.5(3)$ & $2.2(3)$ & $5.9(4)$ & $<0.5$ & $0.9(2)$ & $6.5(4)$ & $2.6(4)$ & $20(1)$ & $3.8(5)$ & $10^{12}$ \\
\hline $\mathrm{CCS}$ & & $8.9(6)$ & $1.1(4)$ & $0.9(5)$ & $4.3(10)$ & & $\gtrsim 1.2$ & $6.2(4)$ & $4.0(10)$ & $22(1)$ & $7.9(12)$ & $10^{11}$ \\
\hline $\mathrm{HCS}^{+}$ & & $8(1)$ & 3(1) & & $5.6(13)$ & & & $2.5(7)$ & $\$ 9.7$ & $36(3)$ & & $10^{11}$ \\
\hline $\mathrm{H}_{2} \mathrm{CS}$ & & $11(2)$ & $1.7(14)$ & & $<3.1$ & & & $9.9(11)$ & $\lesssim 8$ & $23(4)$ & $\lesssim 0.9$ & $10^{11}$ \\
\hline $\mathrm{SiO}$ & & $\geq 1.3$ & & $1.4(1)^{\dagger}$ & $3.9(2)$ & $<0.2$ & $0.21(9)^{\dagger}$ & $0.66(7)$ & $1 . \tilde{4(1)}$ & $20(1)$ & $4.2(2)$ & $10^{12}$ \\
\hline${ }^{29} \mathrm{SiO}$ & & $1.1(4)$ & & $0.4(3)^{\dagger}$ & $2.1(6)$ & & & & & $14(1)$ & $3.3(4)$ & $10^{11}$ \\
\hline${ }^{30} \mathrm{SiO}$ & & $0.7(3)$ & & & & & & & & & & $10^{11}$ \\
\hline$l-\mathrm{C}_{3} \mathrm{H}$ & & $6(1)$ & $2.6(9)$ & $2.8(6)$ & $6.7(13)$ & & & $\lesssim 11$ & $16(3)$ & $26(2)$ & $7(2)$ & $10^{11}$ \\
\hline$l-\mathrm{C}_{3} \mathrm{H}^{+}$ & & $1.4(8)$ & & & $2.6(8)$ & & $1.6(6)$ & & & $6(2)$ & $1.3(4)$ & $10^{11}$ \\
\hline
\end{tabular}

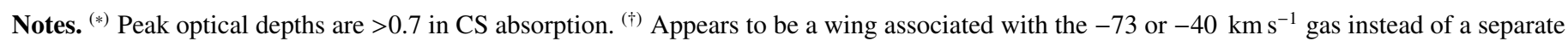
component. 
A\&A 610, A10 (2018)

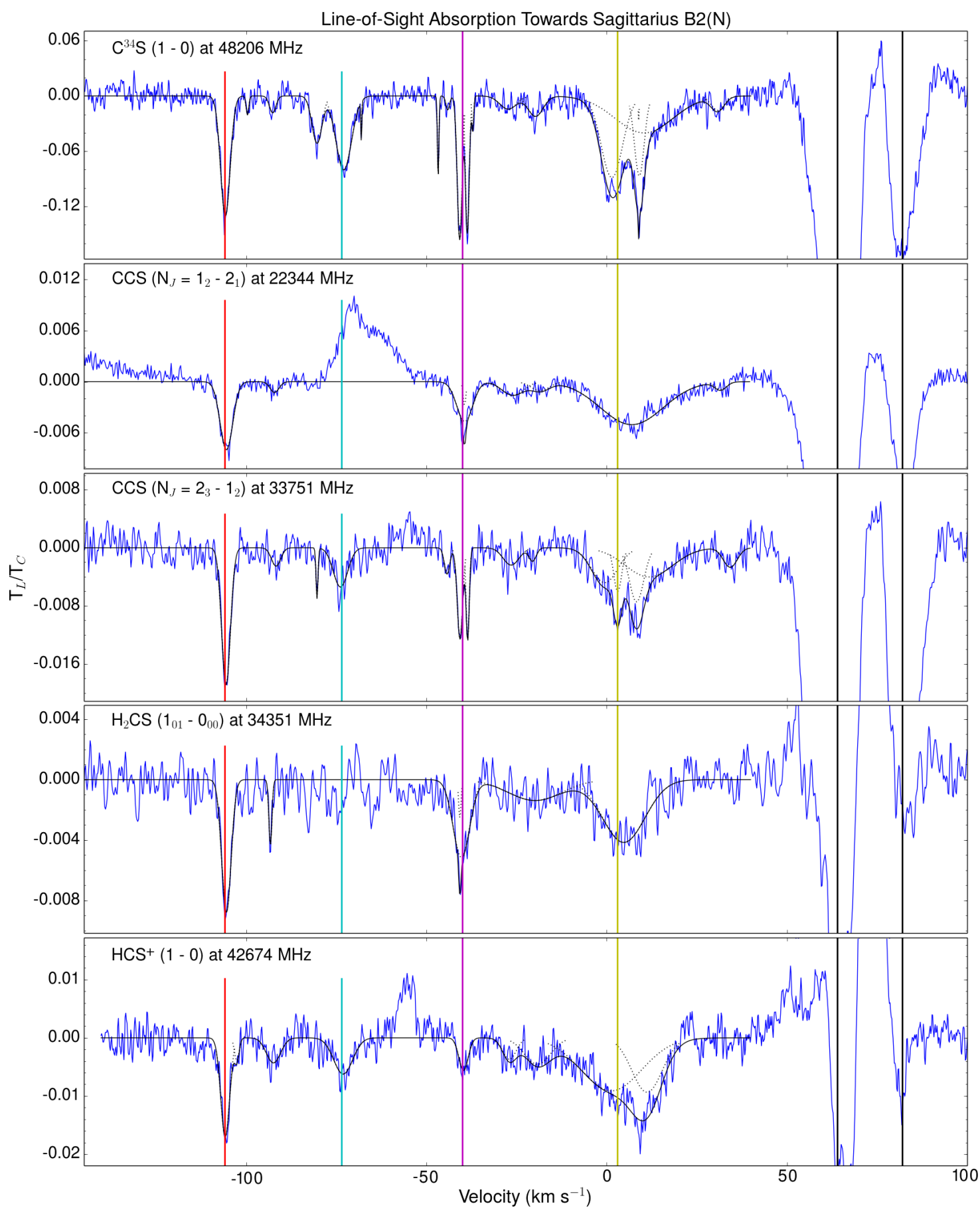

Fig. A.5. Absorption profiles of CS-bearing species are overlaid by the best fit Gaussian components (black dotted line) and the sum of best-fit Gaussians (black solid line). Black vertical lines indicate the velocities of line absorption by Sgr B2 at +64 and $+82 \mathrm{~km} \mathrm{~s}^{-1}$, and colored lines are located at $-106,-73.5,-40$, and $+3 \mathrm{~km} \mathrm{~s}^{-1}$. 
J. F. Corby et al.: The molecular chemistry of diffuse and translucent clouds in the line-of-sight to Sgr B2

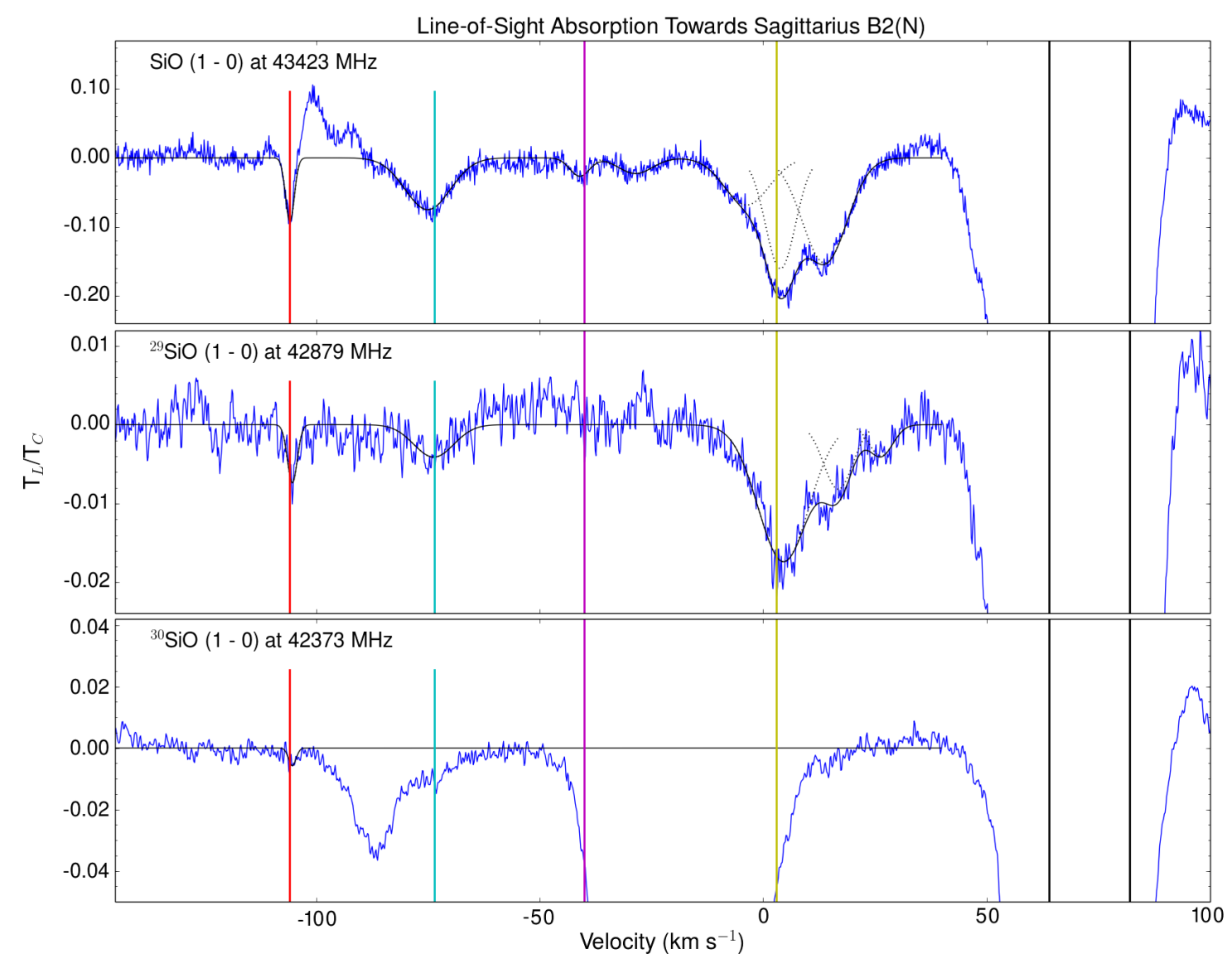

Fig. A.6. Absorption profiles of $\mathrm{SiO}$ isotopologues are overlaid by the best fit Gaussian components (black dotted line) and the sum of best-fit Gaussians (black solid line). Black vertical lines indicate the velocities of line absorption by Sgr B2 at +64 and $+82 \mathrm{~km} \mathrm{~s}^{-1}$, and colored lines are located at $-106,-73.5,-40$, and $+3 \mathrm{~km} \mathrm{~s}^{-1}$. The profile of ${ }^{30} \mathrm{SiO}$ includes a single detected component at $-106 \mathrm{~km} \mathrm{~s}^{-1}$ due to blending with the line-of-sight absorption profile of $\mathrm{NH}_{2} \mathrm{CHO}$. 


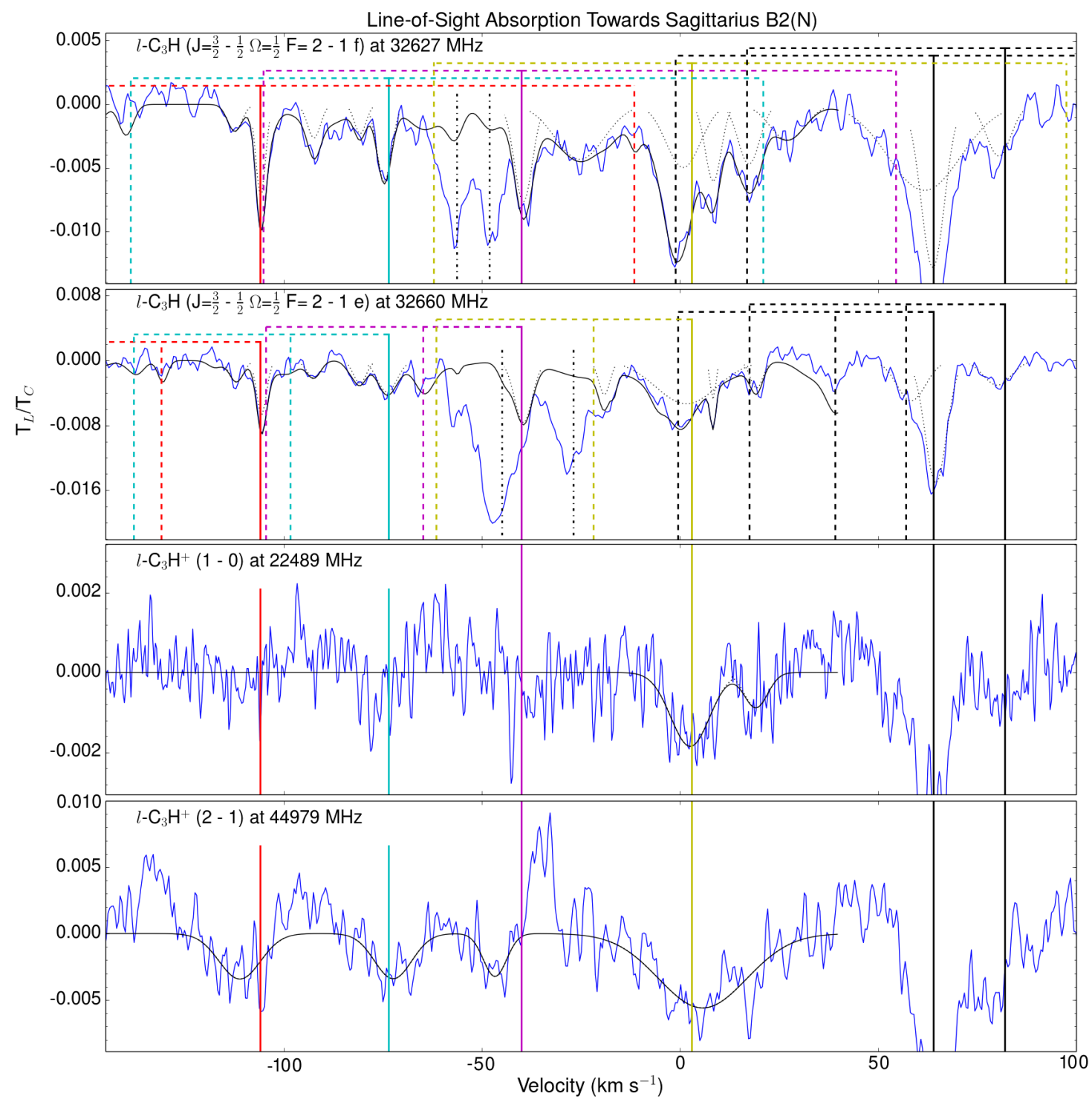

Fig. A.7. Absorption profiles of $l-\mathrm{C}_{3} \mathrm{H}$ and $l-\mathrm{C}_{3} \mathrm{H}^{+}$are overlaid by the best fit Gaussian components (black dotted line) and the sum of best-fit Gaussians (black solid line). Black vertical lines indicate the velocities of line absorption by Sgr B2 at +64 and $+82 \mathrm{~km} \mathrm{~s}^{-1}$, and colored lines are located at $-106,-73.5,-40$, and $+3 \mathrm{~km} \mathrm{~s}^{-1}$. Hyperfine structure for each of these velocity components is indicated by dotted vertical lines of the same color. The data are overlaid by Gaussian components fit to the main hyperfine component shown in black dotted lines, and by the total fit to the profile, which assumes that hyperfine or A/E components are present with the same line shape as the primary component, but with the height scaled by the ratio of the line strengths. In the profile of $l-\mathrm{C}_{3} \mathrm{H}$ at $32627 \mathrm{MHz}$, the black dashed-dotted line marks unidentified transitions that are not consistent with the typical profile of diffuse cloud absorption in this line of sight. In the line profile of $l$ - $\mathrm{C}_{3} \mathrm{H}$ at $32660 \mathrm{MHz}$, the black dashed-dotted line marks a transition of $c i s-\mathrm{CH}_{2} \mathrm{OHCHO}$ at velocities of +64 and $+82 \mathrm{~km} \mathrm{~s}^{-1}$ associated with $\mathrm{Sgr}$ B2. 